\title{
DBU-Promoted Demethoxylative Thioannulation of Alkynyl Oxime Ethers with Sulfur for the Synthesis of Bisisothiazole-4-yl Disulfides
}

Ling Li, ${ }^{\dagger}$ Qian Chen, ${ }^{\dagger}$ Hong-Hui Xu,${ }^{\dagger}$ Xiao-Hong Zhang, ${ }^{* \dagger}$ and Xing-Guo Zhang ${ }^{* \dagger}, *$ ${ }^{\dagger}$ College of Chemistry and Materials Engineering, Wenzhou University, Wenzhou 325035, China; ‡uangxi Key Laboratory of Calcium Carbonate Resources Comprehensive Utilization, Hezhou University, Hezhou 542899, China;

E-Mail:kamenzxh@wzu.edu.cn; zxg@,wzu.edu.cn

\section{Supporting Information \\ List of Contents}

\section{Contents:}

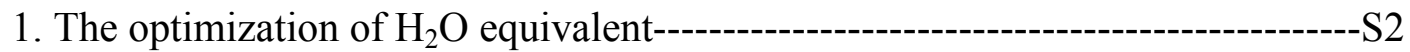

2. NMR Spectra for Starting Reagents 1a-1u--------------------------------------- S3-S13

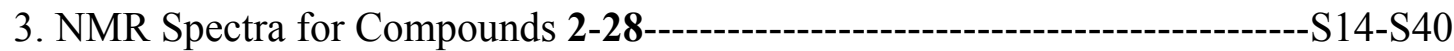

4. X-ray crystal structure and data of compound 3----------------- ---------------S41-S42 
1. Table S1 The optimization of $\mathrm{H}_{2} \mathrm{O}$ equivalent ${ }^{\text {a }}$

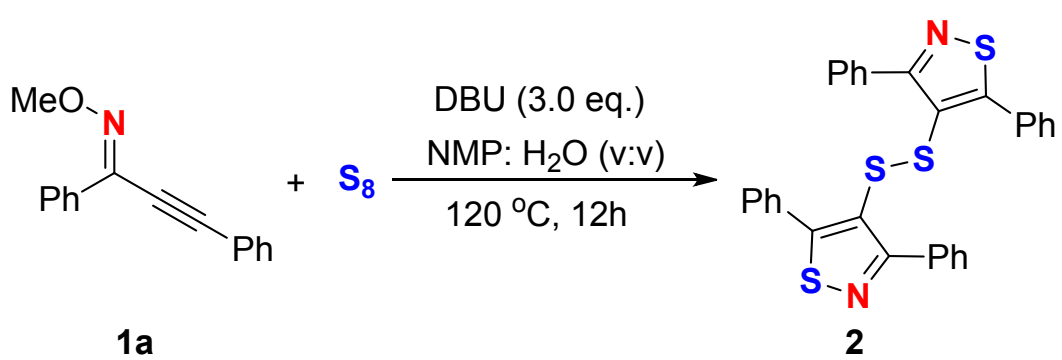

\begin{tabular}{|c|c|c|}
\hline Entry & NMP : $\mathrm{H}_{2} \mathrm{O}(\mathrm{x}: \mathrm{y}, \mathrm{v}: \mathrm{v})$ & Yield (\%) \\
\hline 1 & 0 & 41 \\
\hline 2 & $20: 1$ & 56 \\
\hline 3 & $10: 1$ & 72 \\
\hline 4 & $7: 1$ & 81 \\
\hline 5 & $5: 1$ & 78 \\
\hline 6 & $4: 1$ & \\
\hline
\end{tabular}


2. NMR Spectra for Starting Reagents 1a-1u.

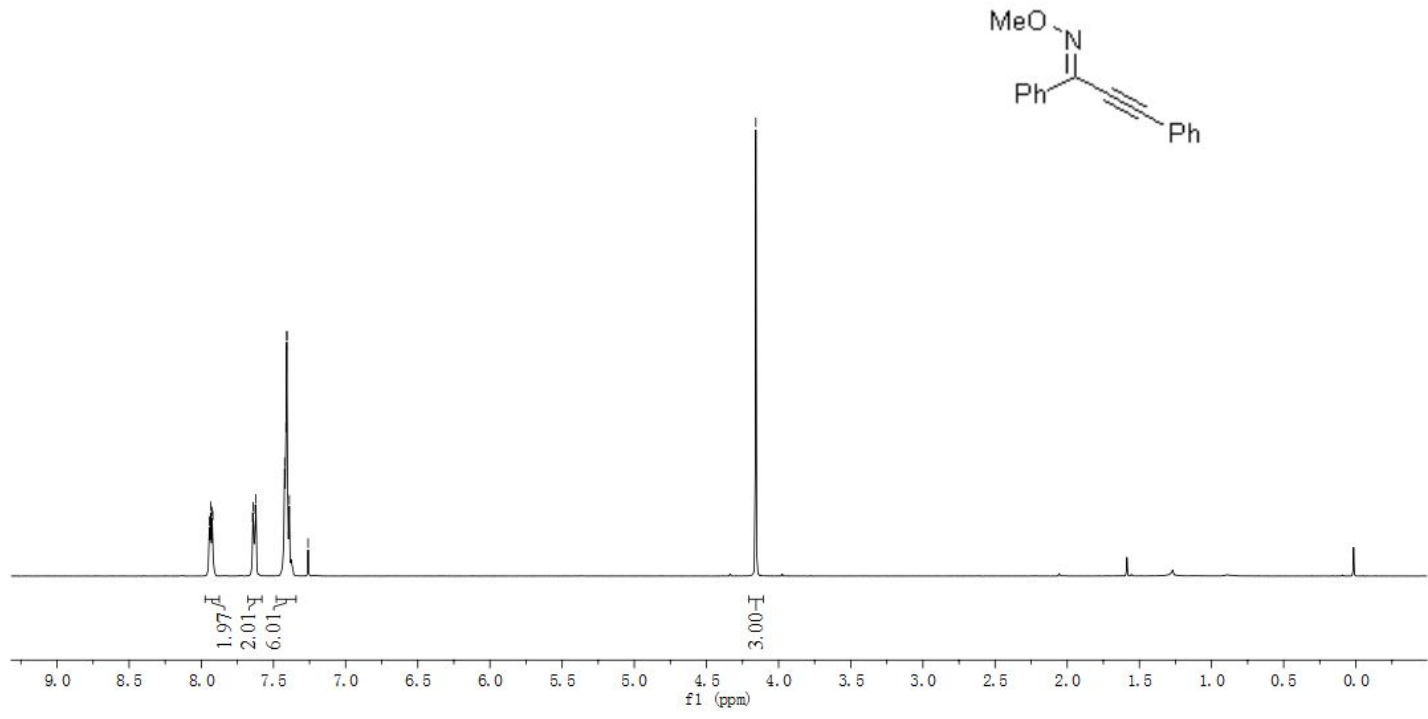

${ }^{1} \mathrm{H}$ NMR $400 \mathrm{MHz}$, in $\mathrm{CDCl}_{3}$ : (E)-1,3-diphenylprop-2-yn-1-one O-methyl oxime (1a)

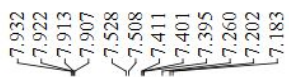

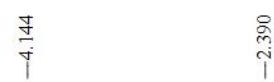
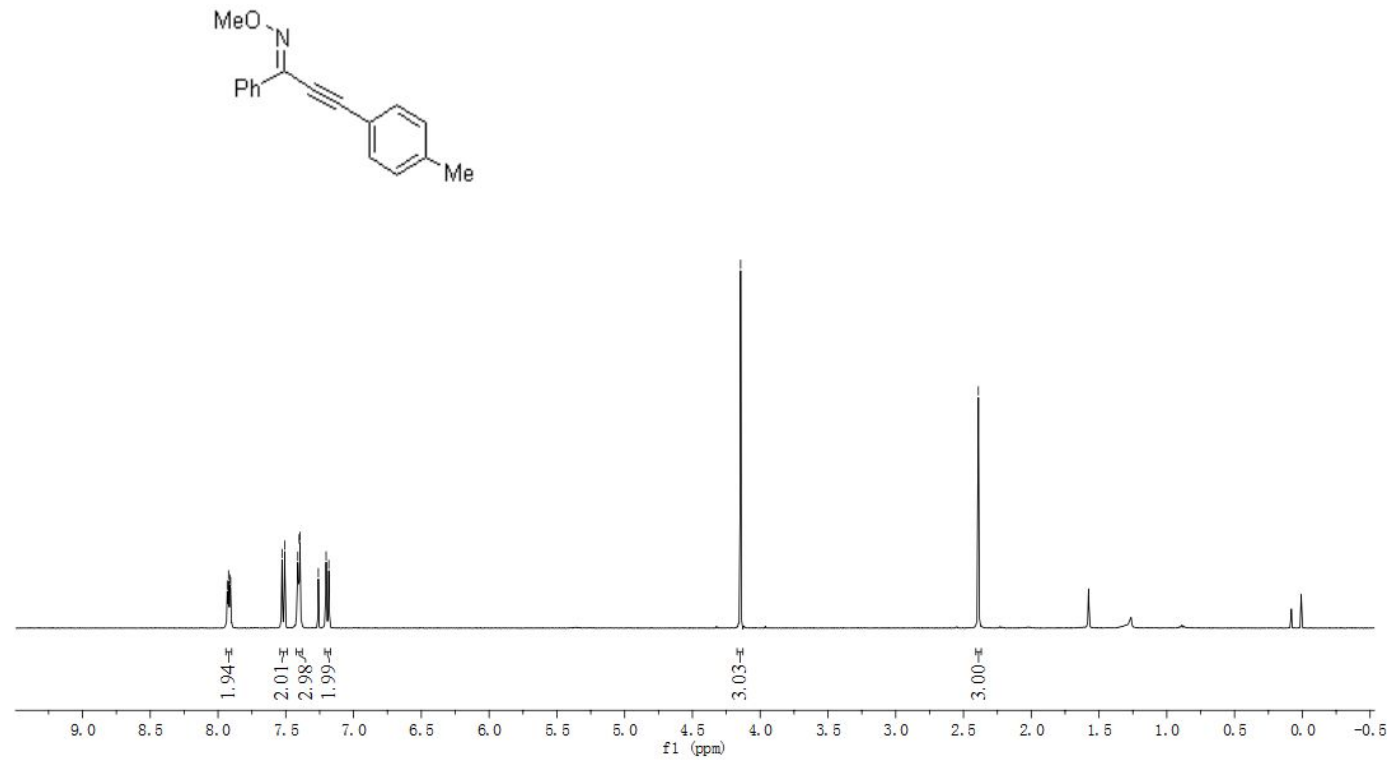

${ }^{1} \mathrm{H}$ NMR 400 MHz, in $\mathrm{CDCl}_{3}$ : (E)-1-phenyl-3-(p-tolyl)prop-2-yn-1-one O-methyl oxime (1b) 


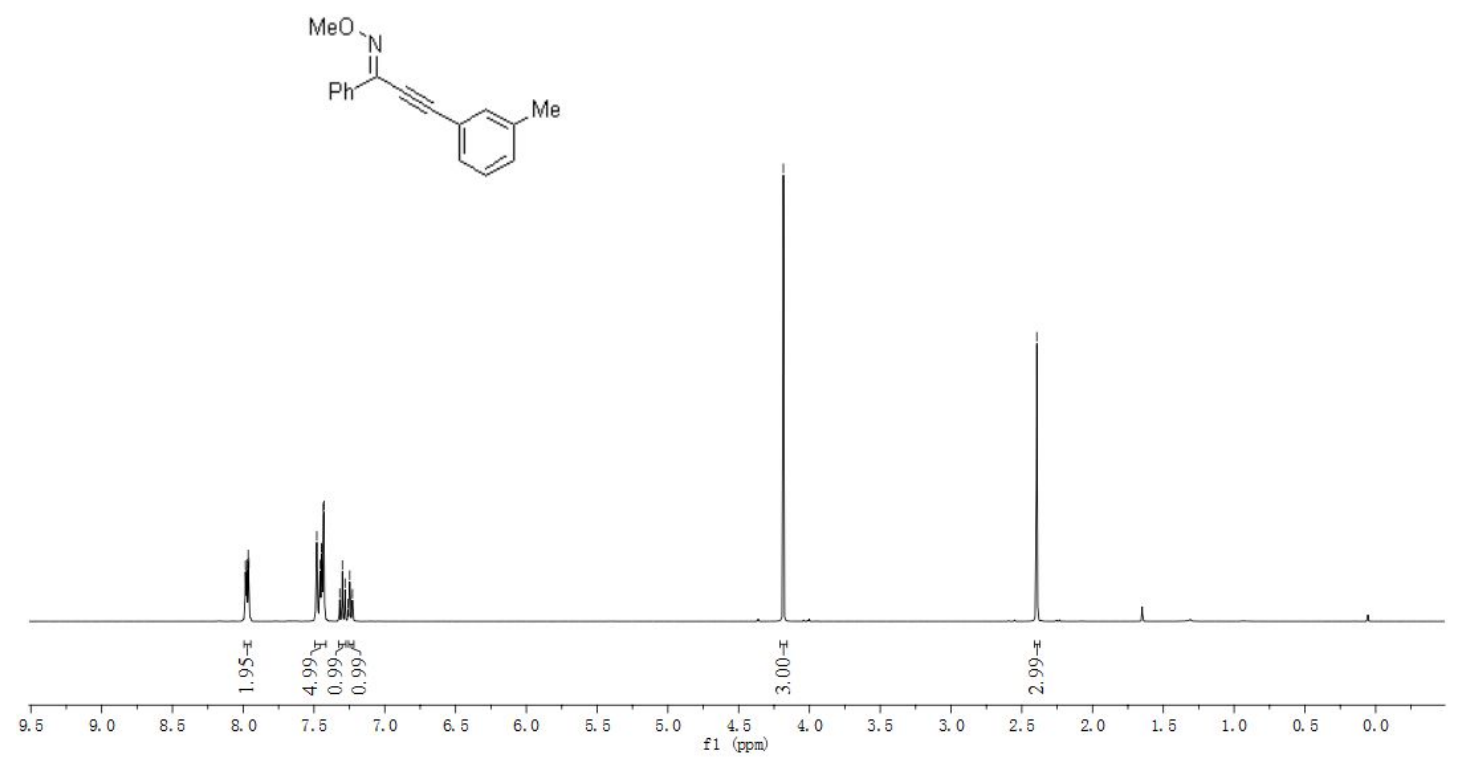

${ }^{1} \mathrm{H}$ NMR $400 \mathrm{MHz}$, in $\mathrm{CDCl}_{3}$ : (E)-1-phenyl-3-(m-tolyl)prop-2-yn-1-one O-methyl oxime (1c)

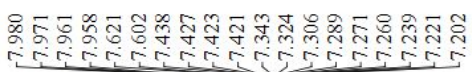
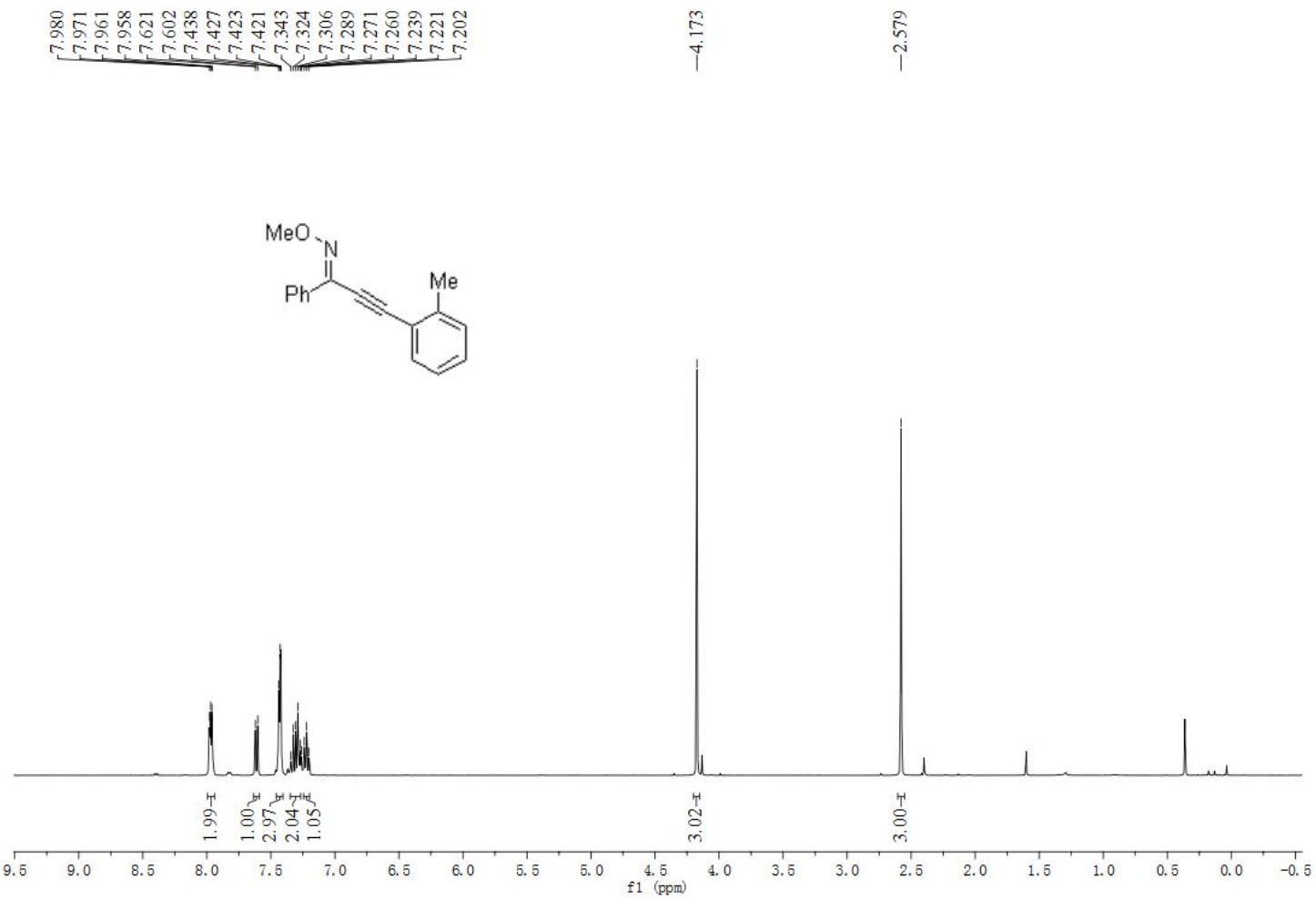

${ }^{1} \mathrm{H}$ NMR $400 \mathrm{MHz}$, in $\mathrm{CDCl}_{3}$ : (E)-1-phenyl-3-(o-tolyl)prop-2-yn-1-one O-methyl oxime (1d) 

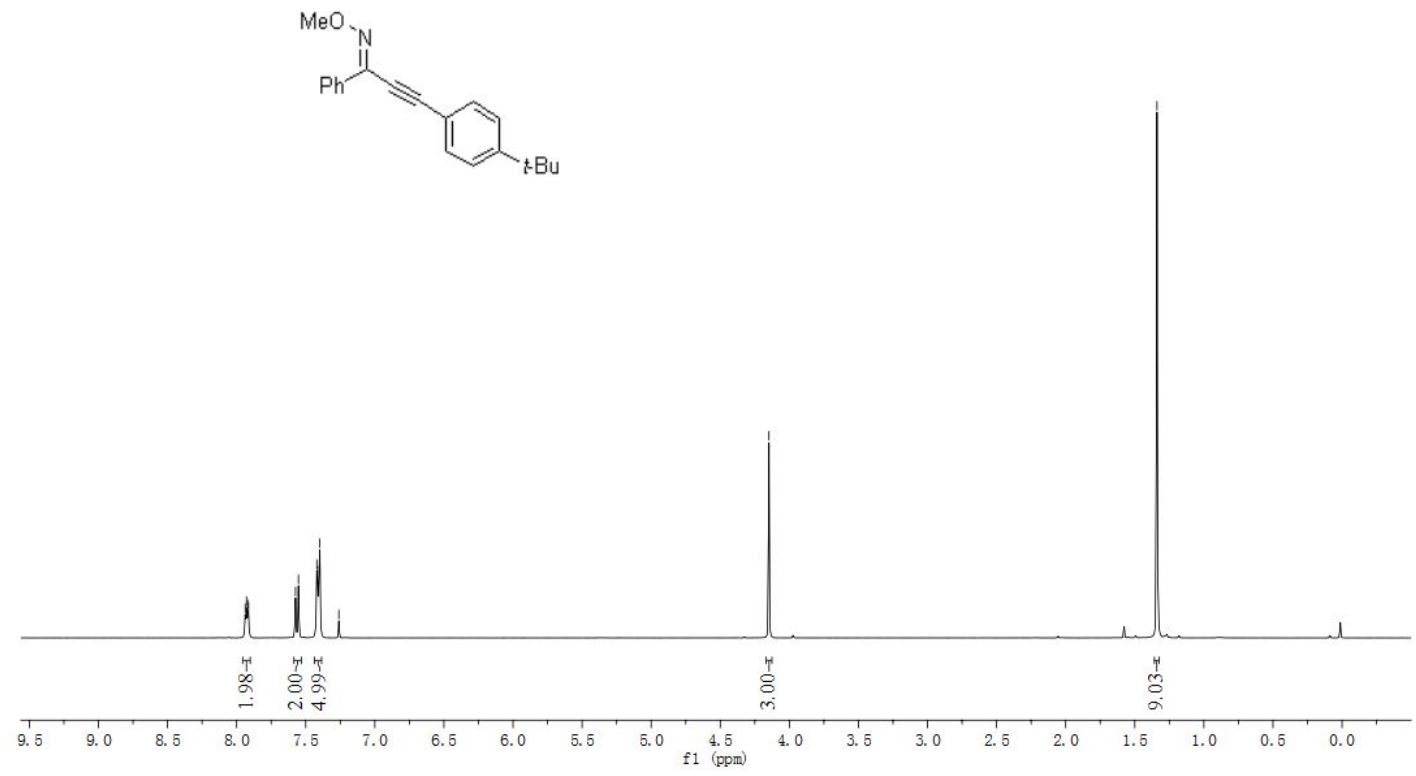

${ }^{1} \mathrm{H}$ NMR $400 \mathrm{MHz}$, in $\mathrm{CDCl}_{3}$ :

(E)-3-(4-(tert-butyl)phenyl)-1-phenylprop-2-yn-1-one O-methyl oxime (1e)

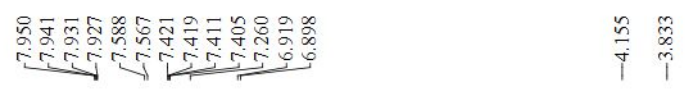

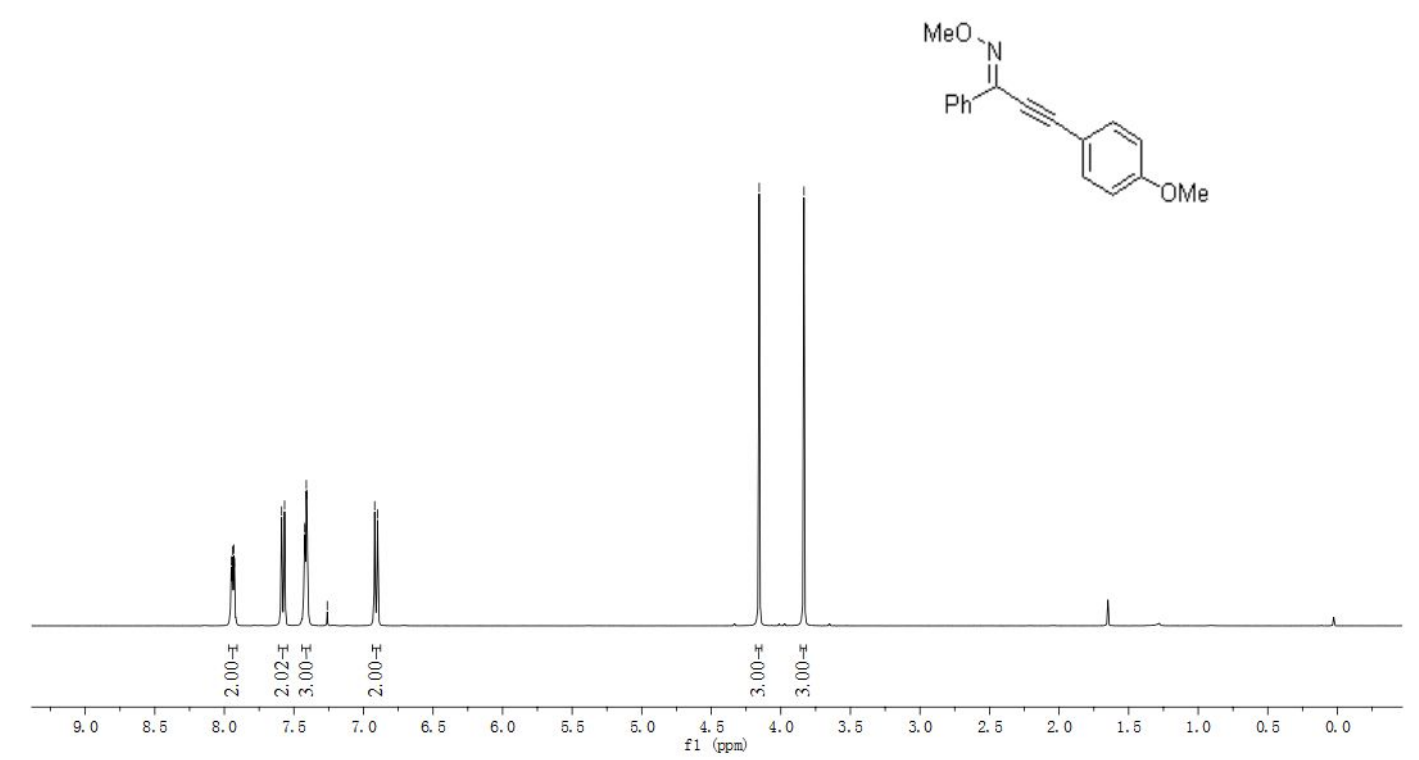

${ }^{1} \mathrm{H}$ NMR $400 \mathrm{MHz}$, in $\mathrm{CDCl}_{3}$ : (E)-3-(4-methoxyphenyl)-1-phenylprop-2-yn-1-one O-methyl oxime (1f) 


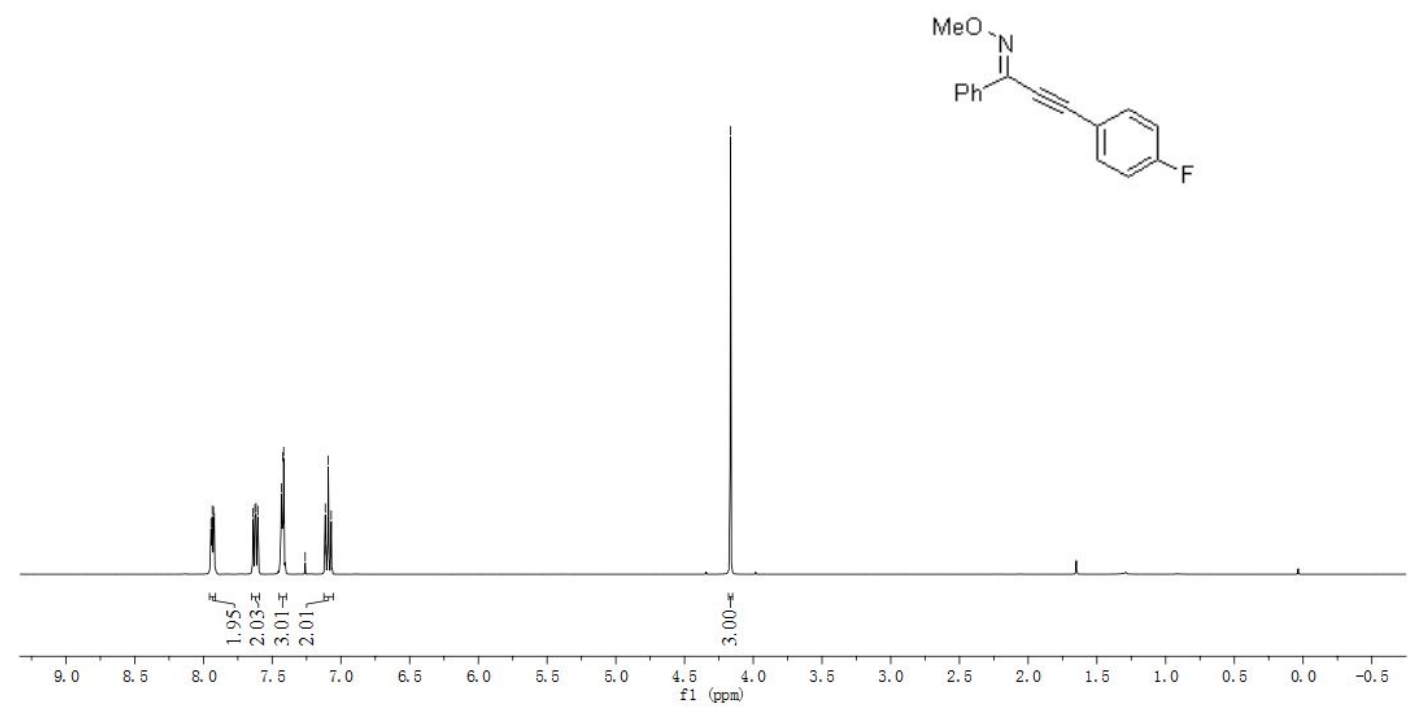

${ }^{1} \mathrm{H}$ NMR $400 \mathrm{MHz}$, in $\mathrm{CDCl}_{3}$ : (E)-3-(4-fluorophenyl)-1-phenylprop-2-yn-1-one

O-methyl oxime (1g)

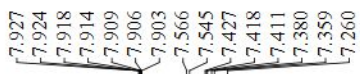

$\frac{\mathscr{b}}{\dot{y}}$

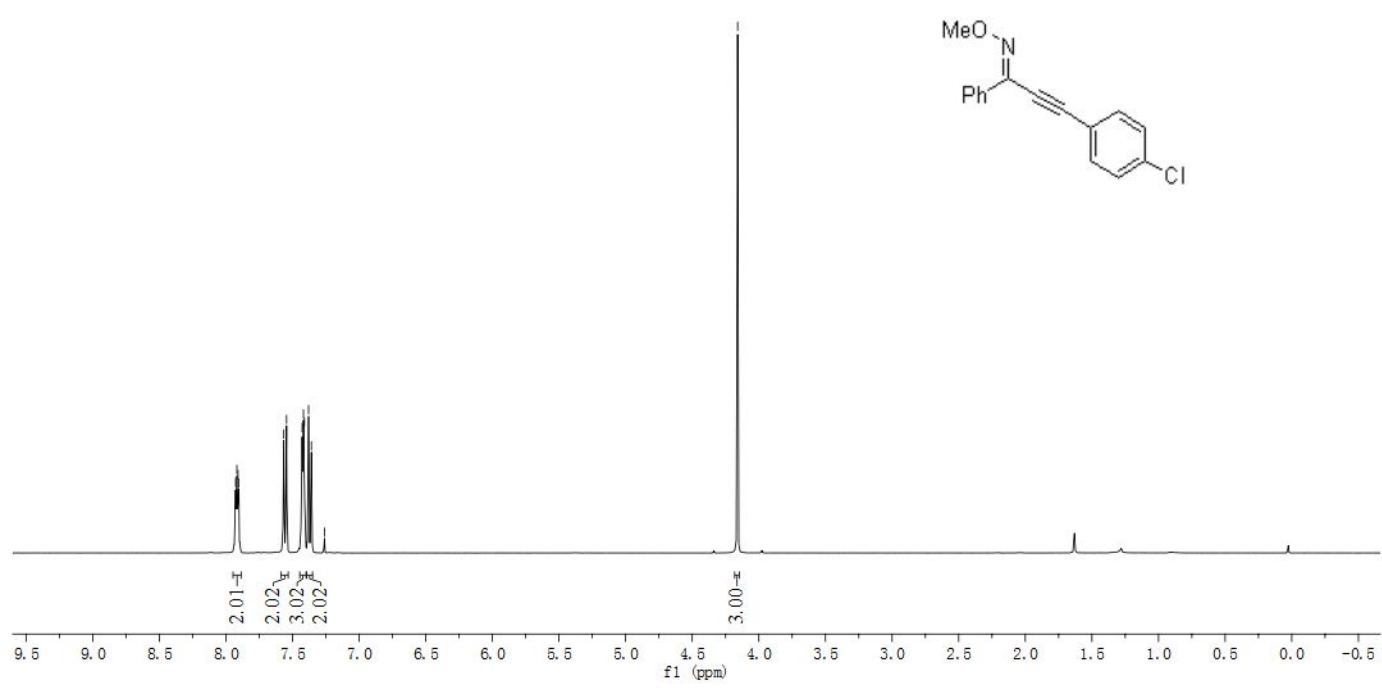

${ }^{1} \mathrm{H}$ NMR $400 \mathrm{MHz}$, in $\mathrm{CDCl}_{3}$ : (E)-3-(4-chlorophenyl)-1-phenylprop-2-yn-1-one

O-methyl oxime (1h) 


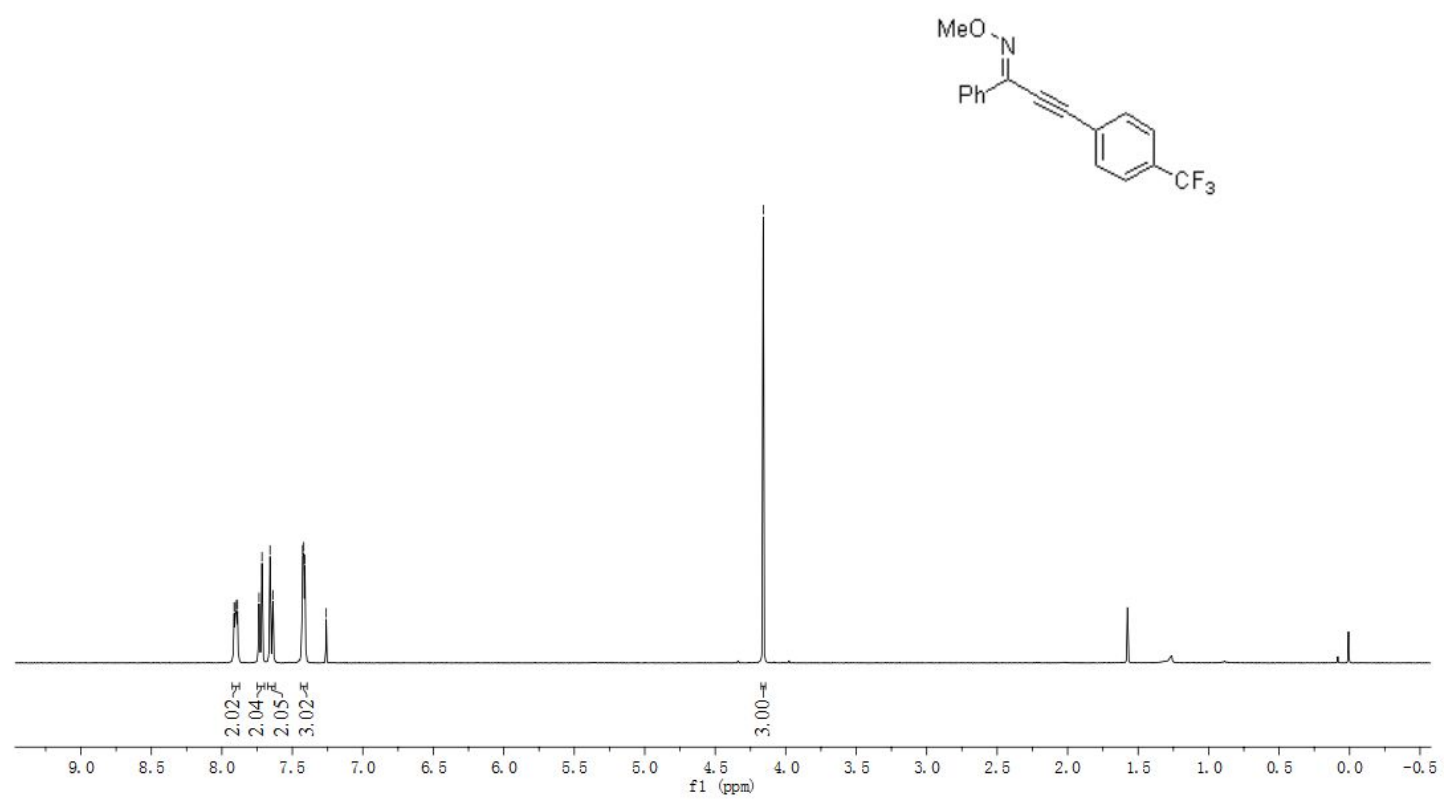

${ }^{1} \mathrm{H}$ NMR $400 \mathrm{MHz}$, in $\mathrm{CDCl}_{3}$ :

(E)-1-phenyl-3-(4-(trifluoromethyl)phenyl)prop-2-yn-1-one O-methyl oxime (1i)

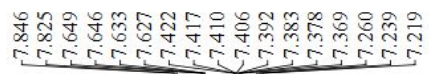

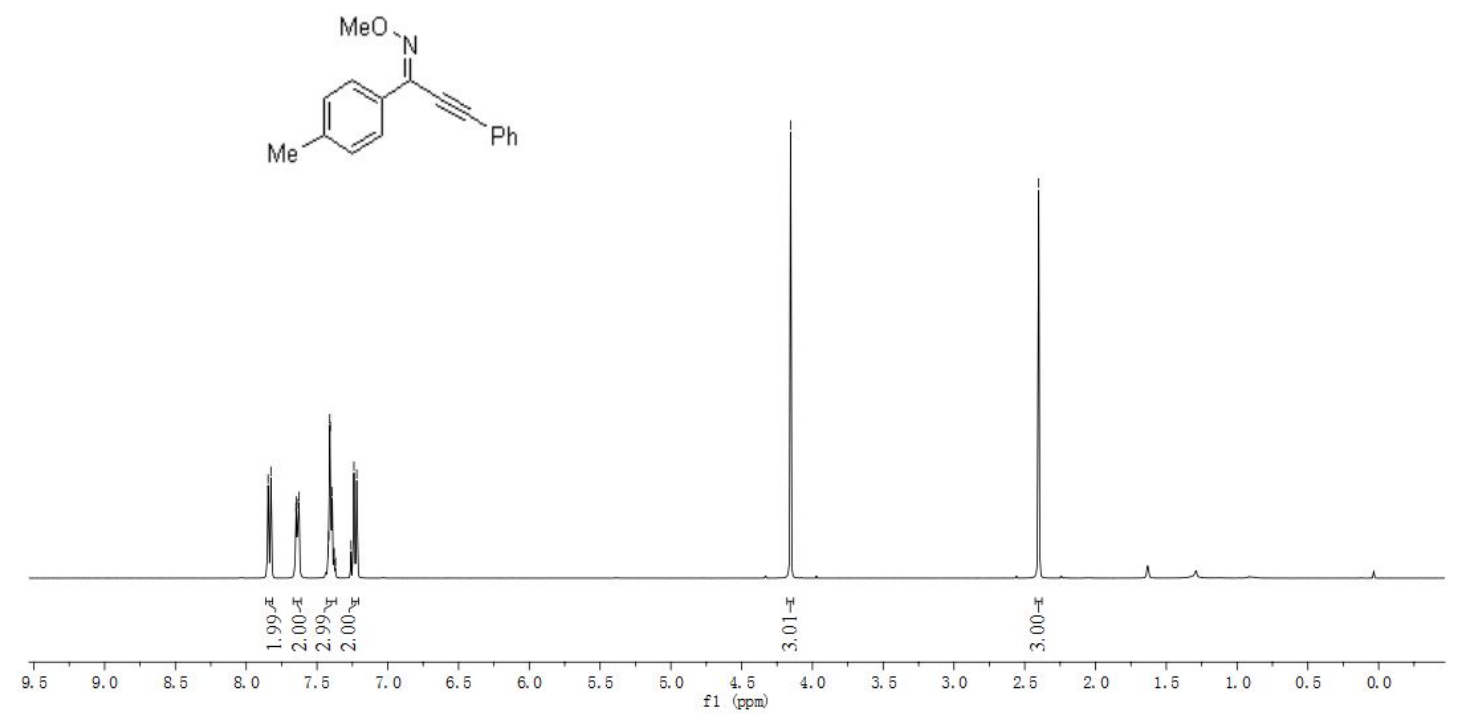

${ }^{1} \mathrm{H}$ NMR $400 \mathrm{MHz}$, in $\mathrm{CDCl}_{3}$ : (E)-3-phenyl-1-(p-tolyl)prop-2-yn-1-one O-methyl oxime (1j) 
<smiles>CO/N=C(/C#Cc1ccccc1)c1ccccc1C</smiles>

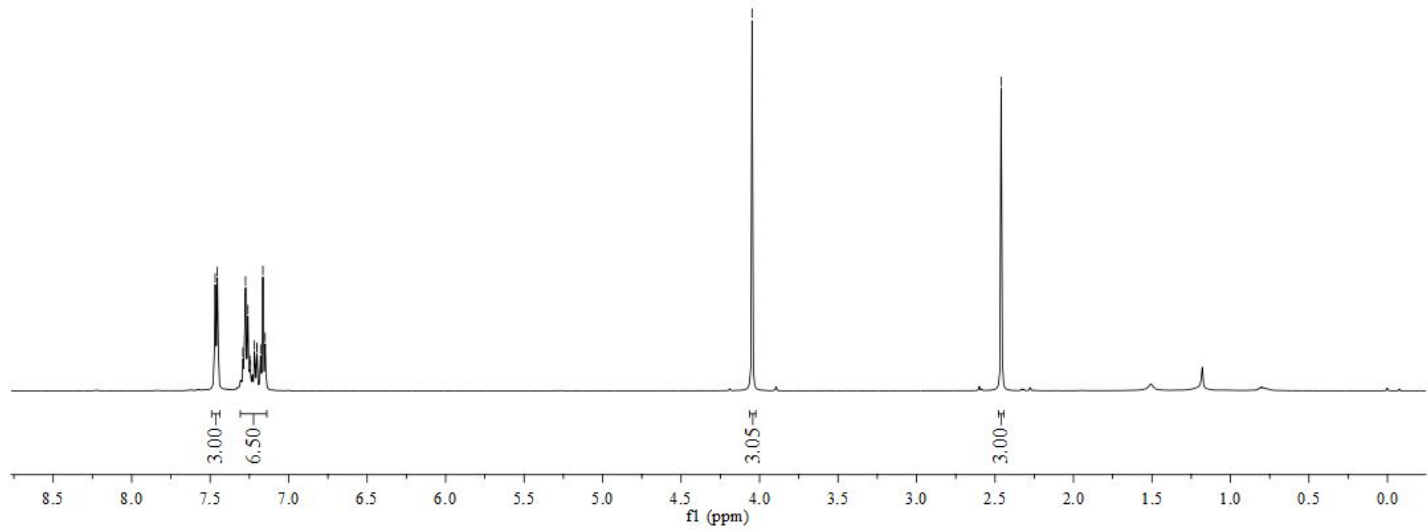

${ }^{1} \mathrm{H}$ NMR $500 \mathrm{MHz}$, in $\mathrm{CDCl}_{3}$ : (Z)-3-phenyl-1-(o-tolyl)prop-2-yn-1-one O-methyl oxime (1k)

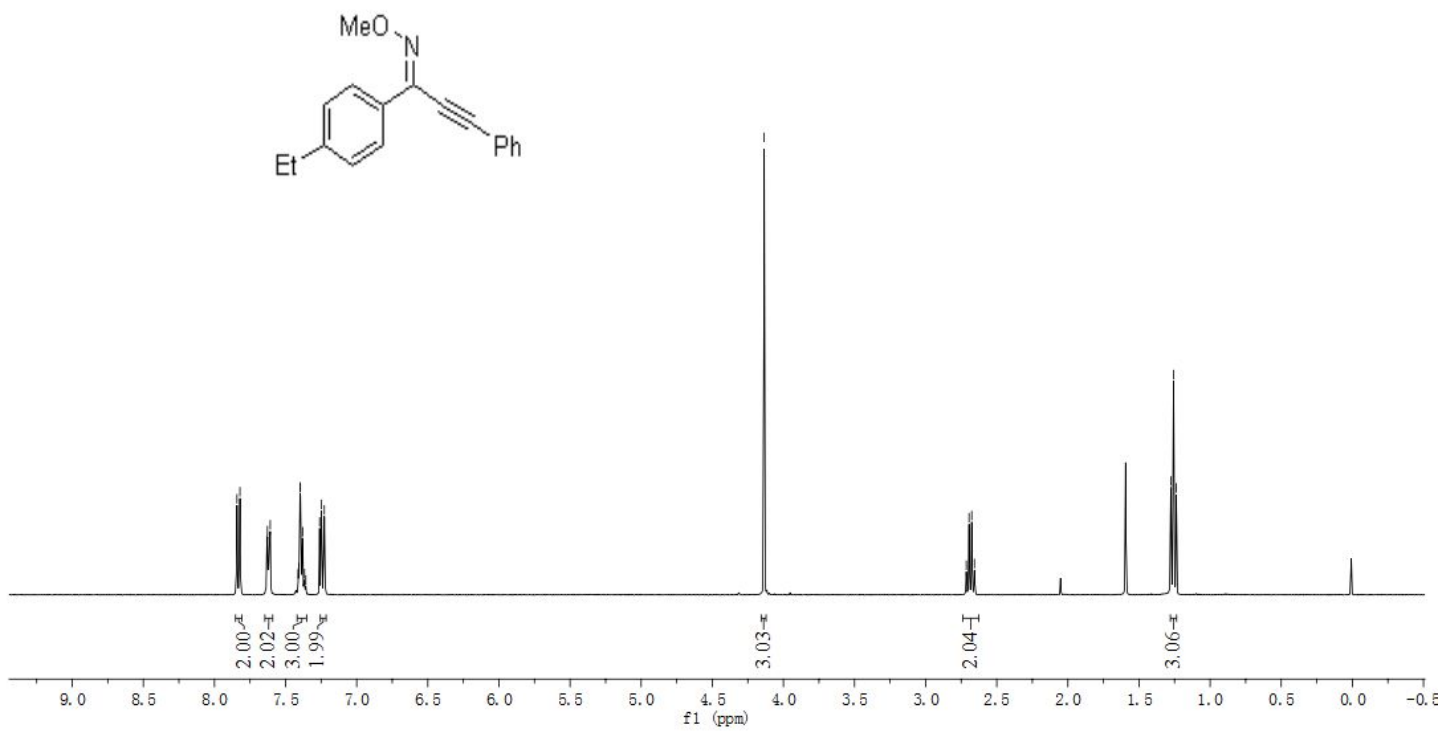

${ }^{1} \mathrm{H}$ NMR $400 \mathrm{MHz}$, in $\mathrm{CDCl}_{3}$ : (E)-1-(4-ethylphenyl)-3-phenylprop-2-yn-1-one

O-methyl oxime (11) 


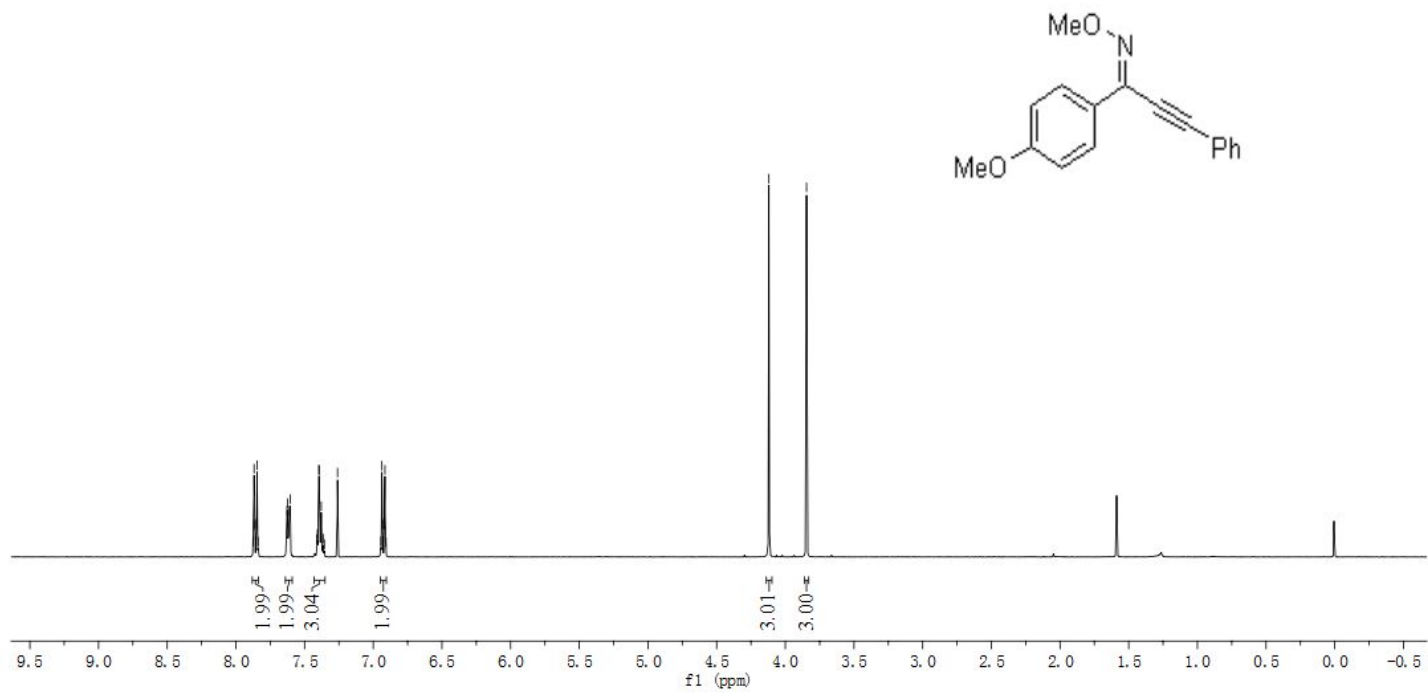

${ }^{1} \mathrm{H}$ NMR $400 \mathrm{MHz}$, in $\mathrm{CDCl}_{3}$ : (E)-1-(4-methoxyphenyl)-3-phenylprop-2-yn-1-one O-methyl oxime (1 m)

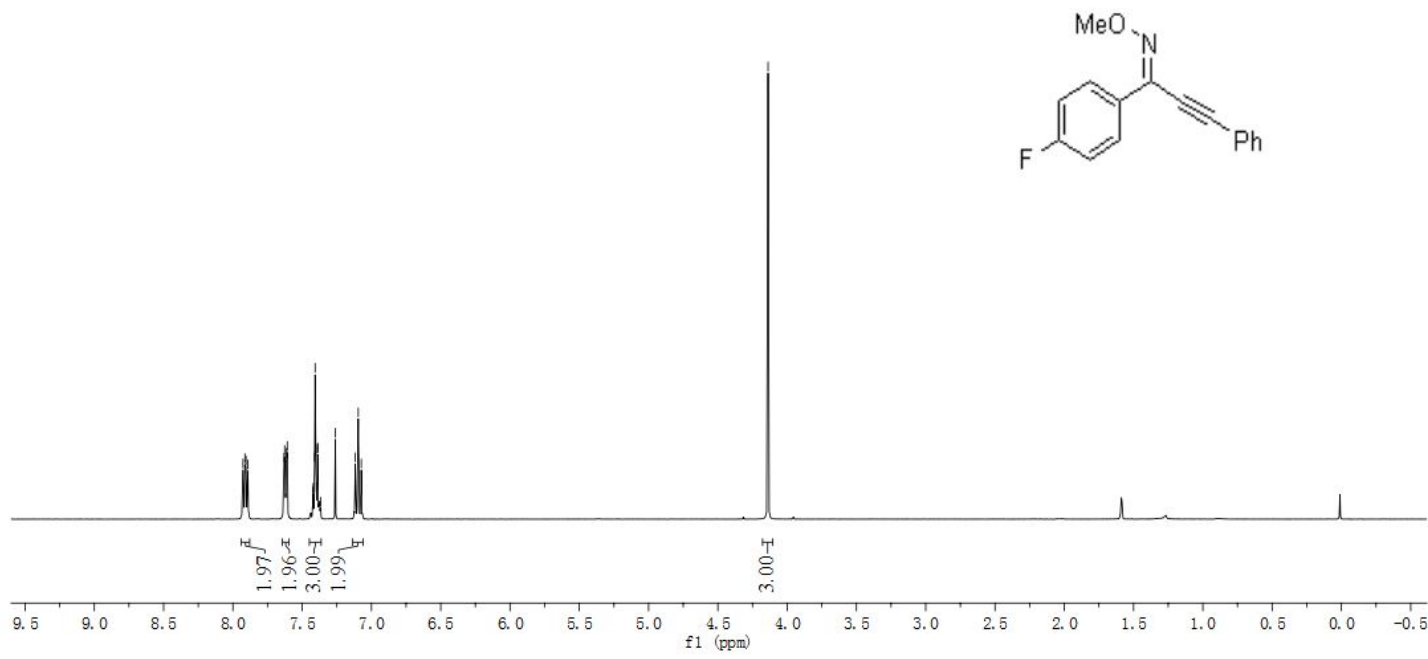

${ }^{1} \mathrm{H}$ NMR $400 \mathrm{MHz}$, in $\mathrm{CDCl}_{3}$ : (E)-1-(4-fluorophenyl)-3-phenylprop-2-yn-1-one

O-methyl oxime (1n) 


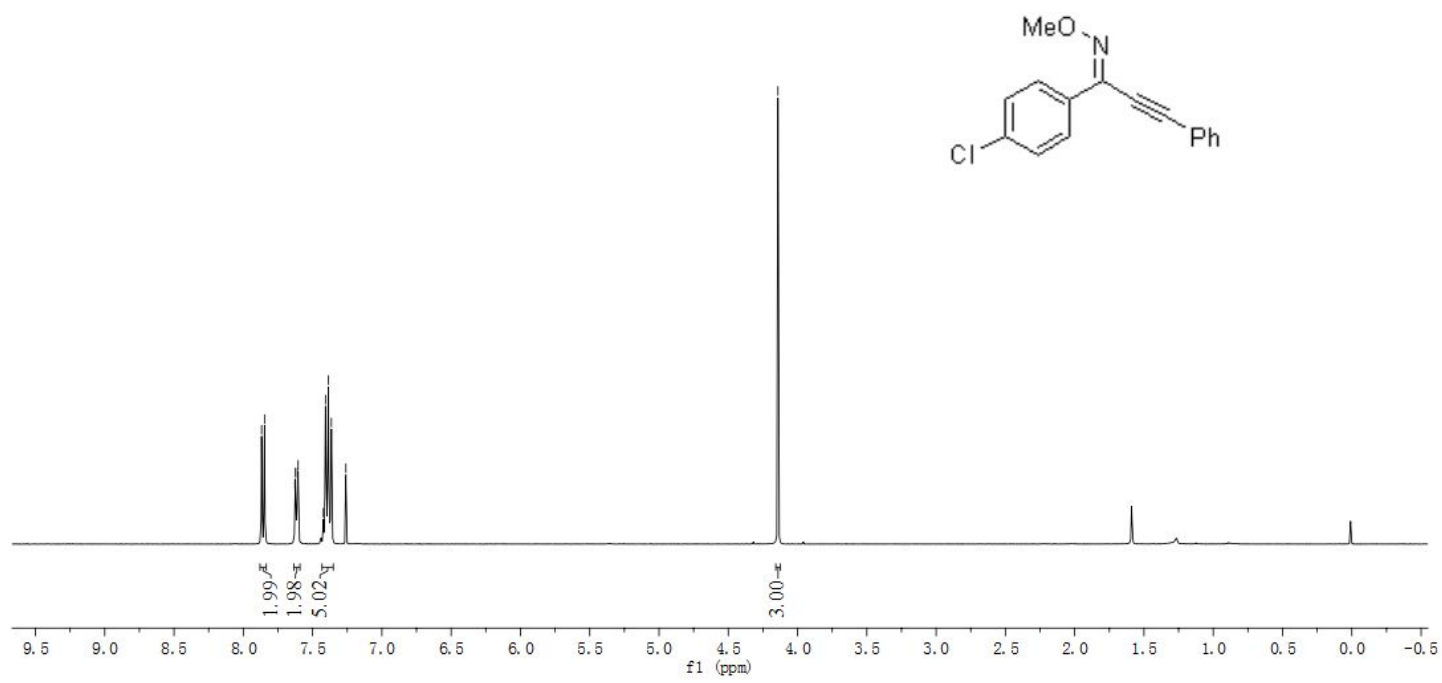

${ }^{1} \mathrm{H}$ NMR $400 \mathrm{MHz}$, in $\mathrm{CDCl}_{3}$ : (E)-1-(4-chlorophenyl)-3-phenylprop-2-yn-1-one

O-methyl oxime (10)

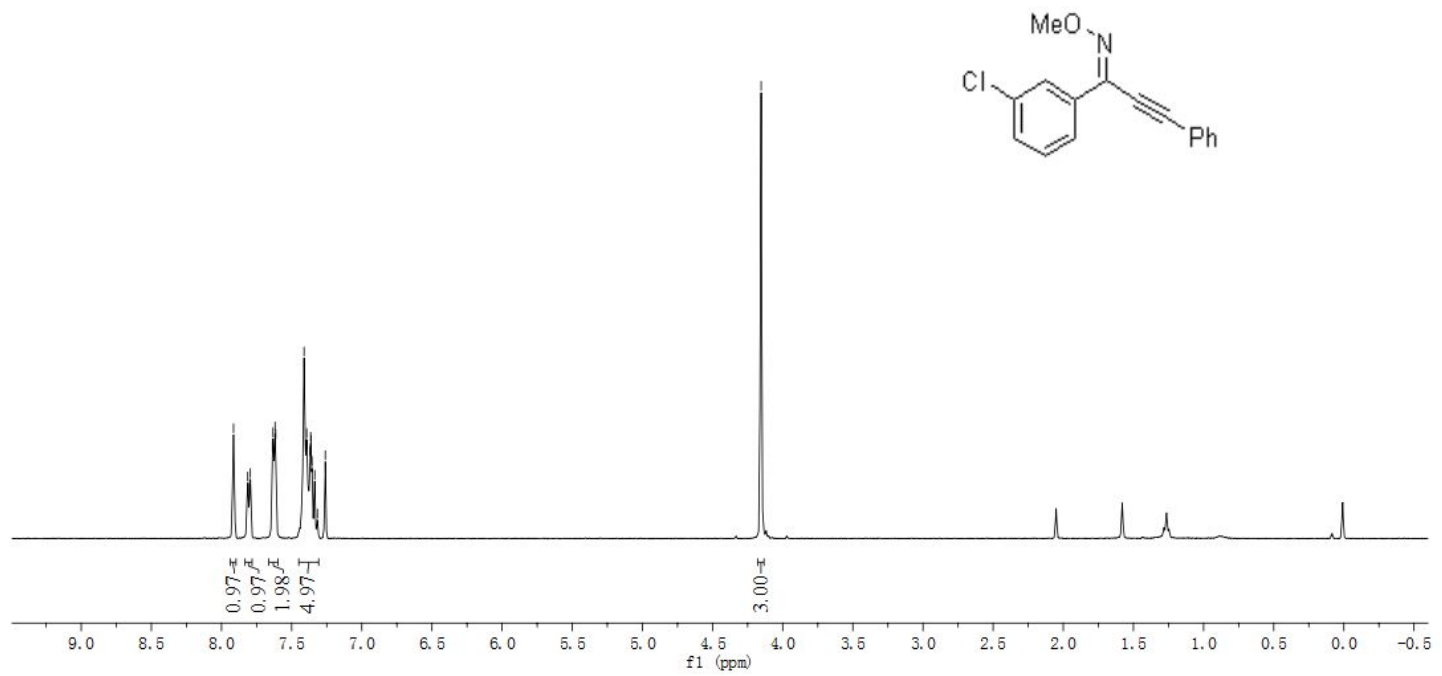

${ }^{1} \mathrm{H}$ NMR $400 \mathrm{MHz}$, in $\mathrm{CDCl}_{3}$ : (E)-1-(3-chlorophenyl)-3-phenylprop-2-yn-1-one

O-methyl oxime (1p) 


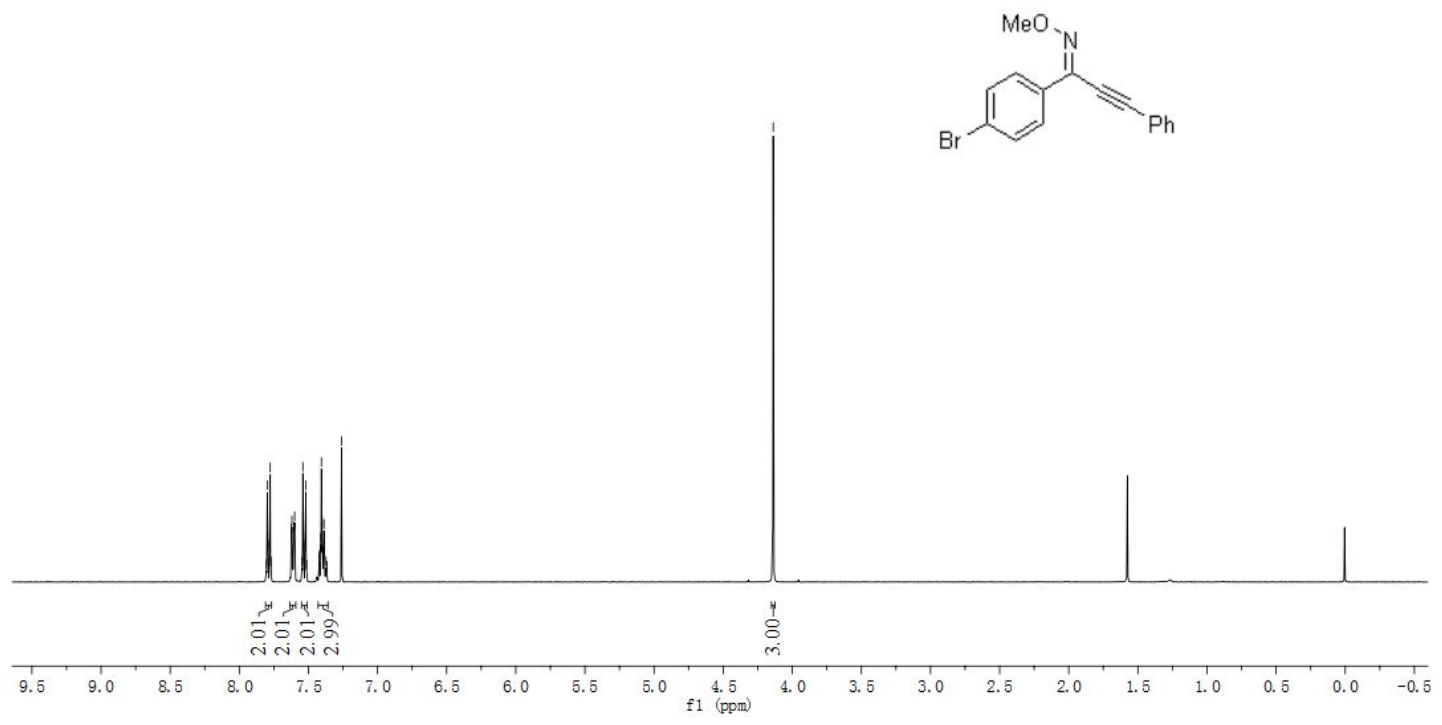

${ }^{1} \mathrm{H}$ NMR 400 MHz, in $\mathrm{CDCl}_{3}$ : (E)-1-(4-bromophenyl)-3-phenylprop-2-yn-1-one

O-methyl oxime (1q)
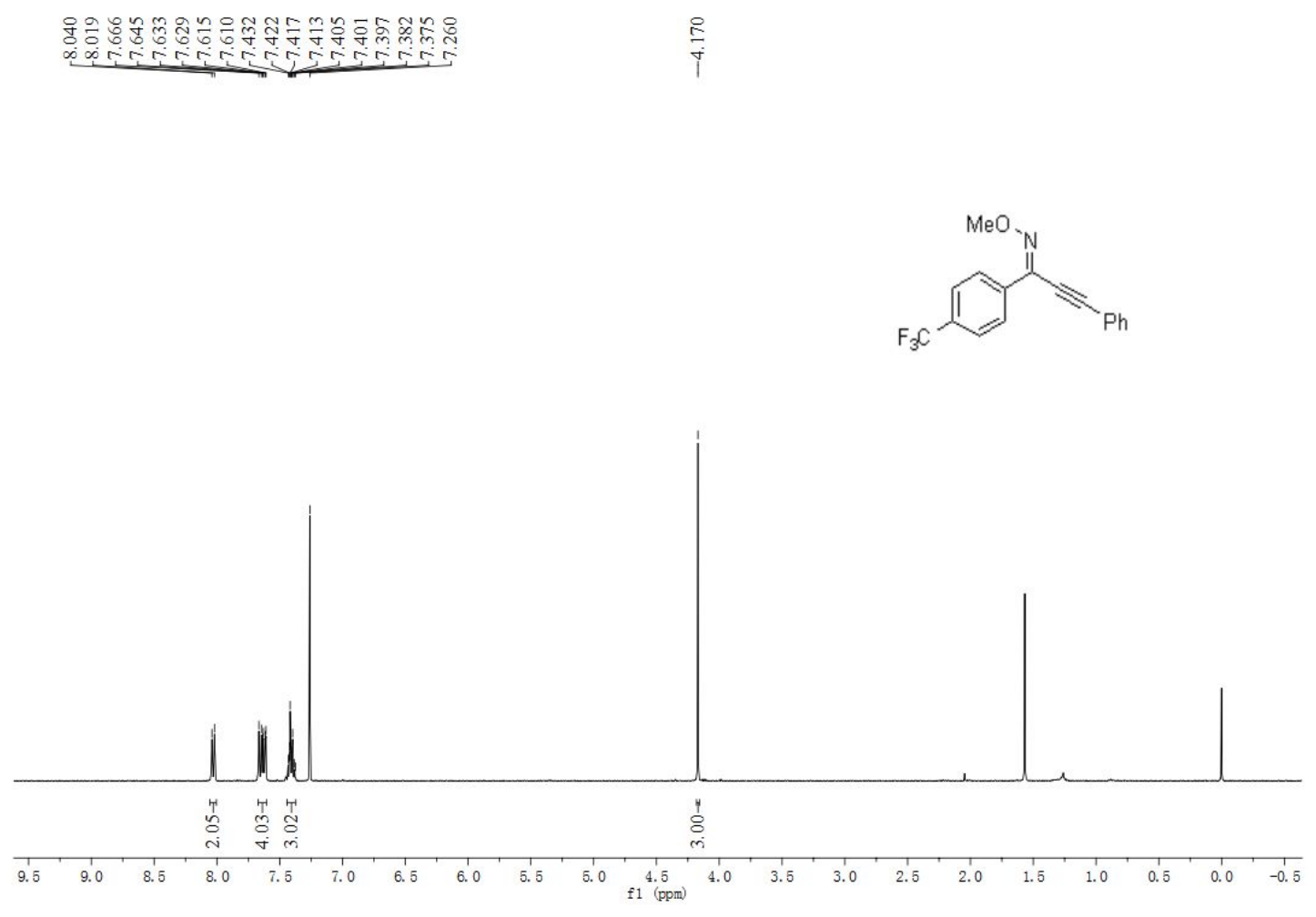

${ }^{1} \mathrm{H}$ NMR $400 \mathrm{MHz}$, in $\mathrm{CDCl}_{3}$ :

(E)-3-phenyl-1-(4-(trifluoromethyl)phenyl)prop-2-yn-1-one O-methyl oxime (1r) 


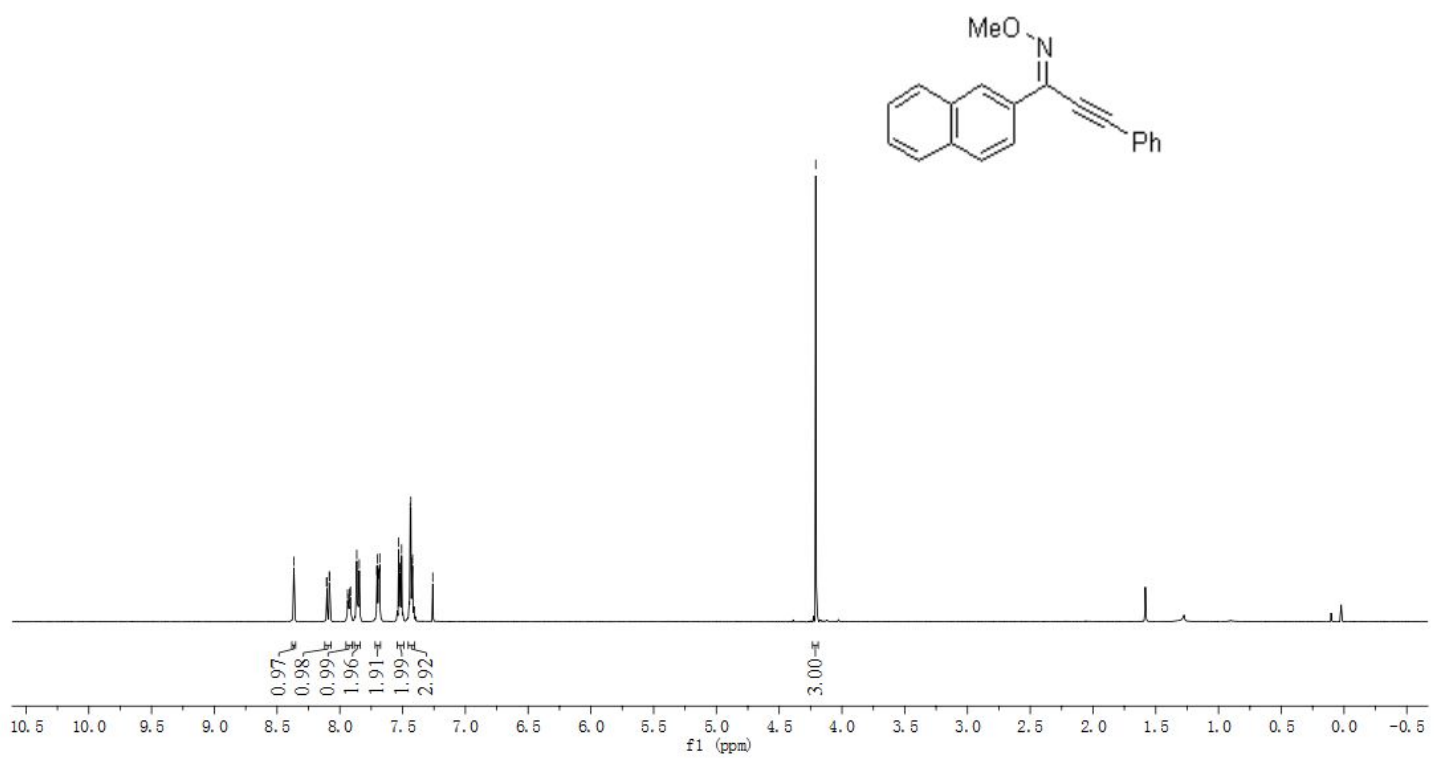

${ }^{1} \mathrm{H}$ NMR $400 \mathrm{MHz}$, in $\mathrm{CDCl}_{3}$ : (E)-1-(naphthalen-2-yl)-3-phenylprop-2-yn-1-one

O-methyl oxime (1s)

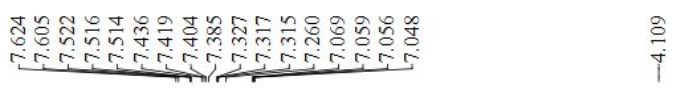

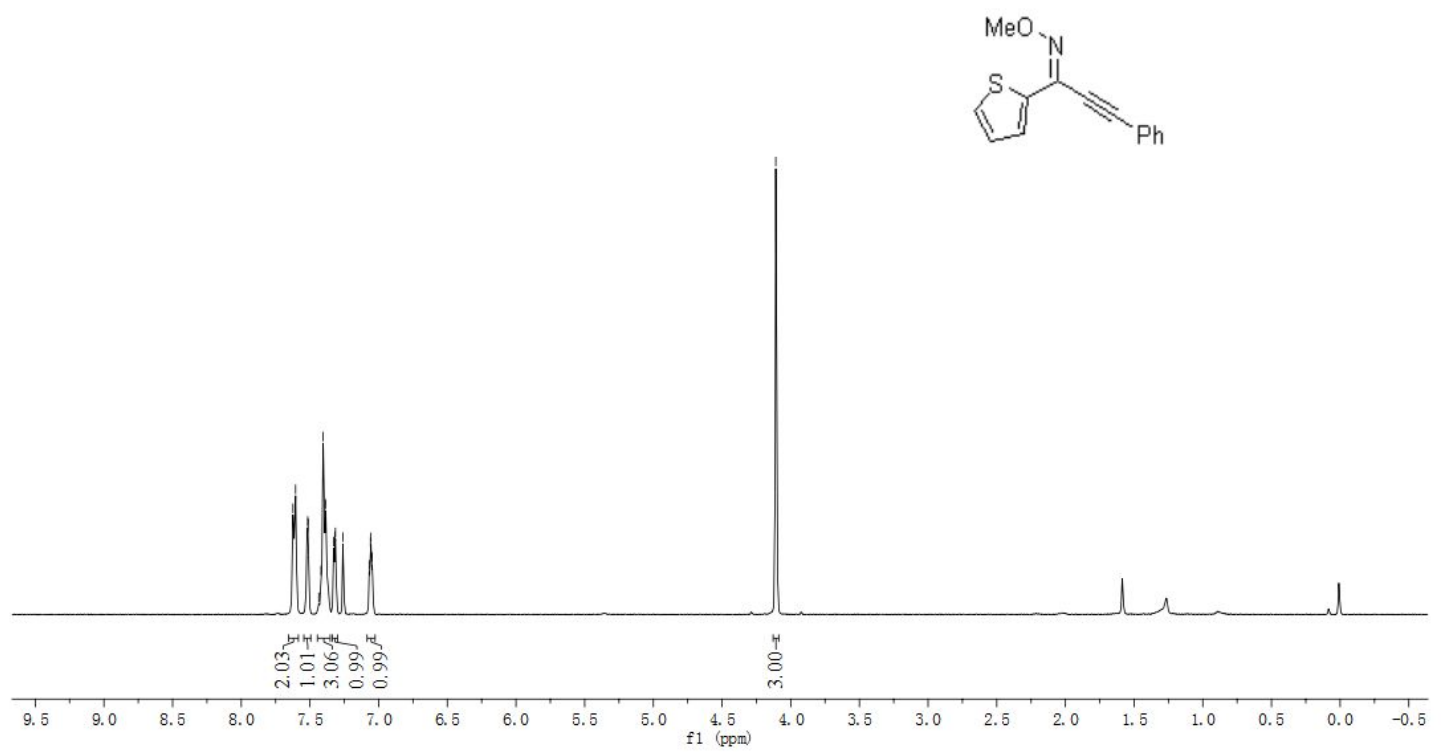

${ }^{1} \mathrm{H}$ NMR $400 \mathrm{MHz}$, in $\mathrm{CDCl}_{3}$ : (Z)-3-phenyl-1-(thiophen-2-yl)prop-2-yn-1-one O-methyl oxime (1t) 
<smiles>CO/N=C(/C#Cc1ccccc1)C1CCCCC1</smiles>

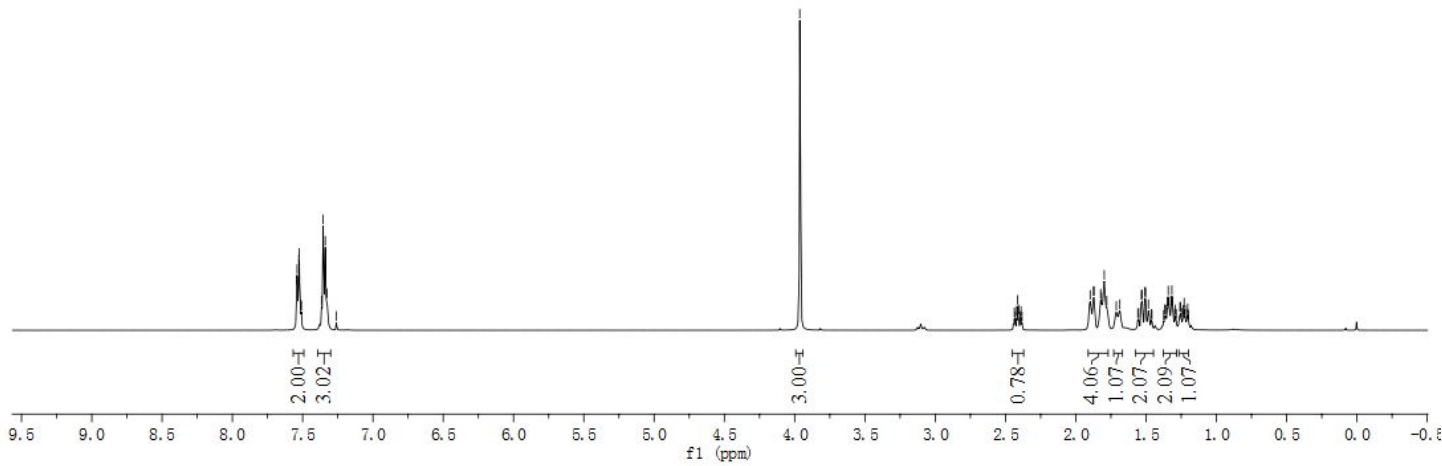

${ }^{1} \mathrm{H}$ NMR $500 \mathrm{MHz}$, in $\mathrm{CDCl}_{3}$ : (E)-1-cyclohexyl-3-phenylprop-2-yn-1-one

O-methyl oxime (1u) 
3. NMR Spectra for All Compounds 2-28.

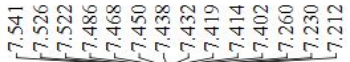
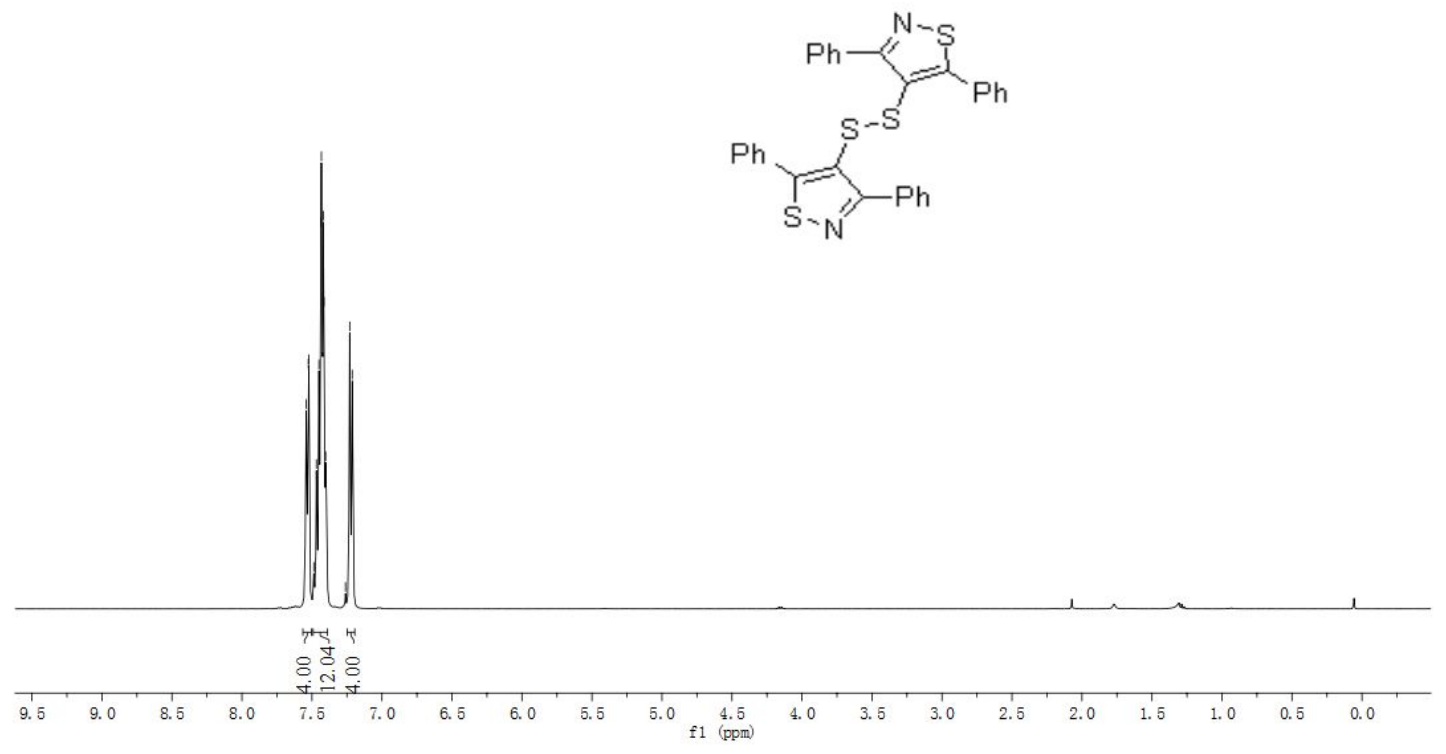

${ }^{1} \mathrm{H}$ NMR $400 \mathrm{MHz}$, in $\mathrm{CDCl}_{3}$ : 1,2-bis(3,5-diphenylisothiazol-4-yl)disulfane (2)
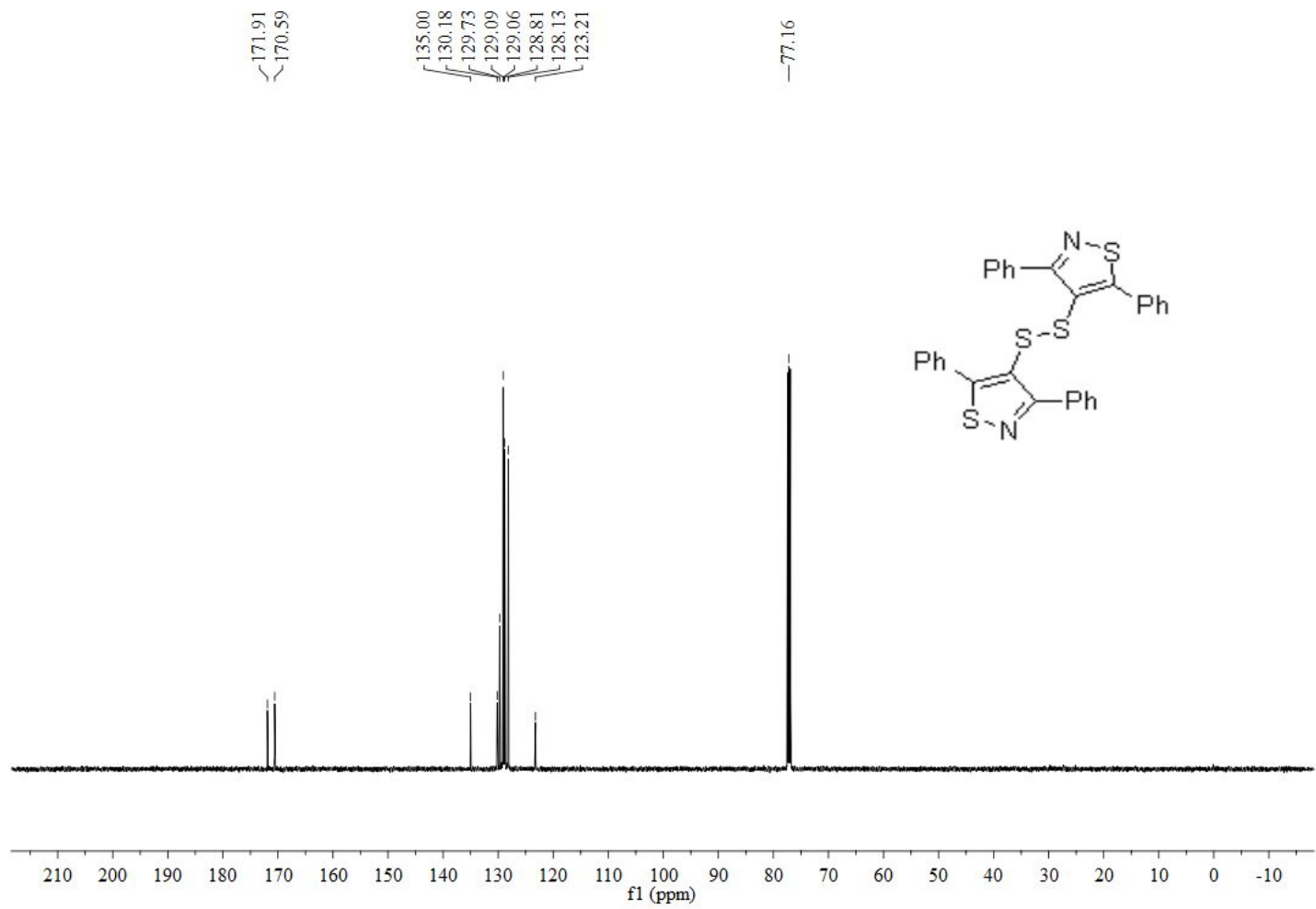

${ }^{13} \mathrm{C}$ NMR $125 \mathrm{MHz}$, in $\mathrm{CDCl}_{3}$ : 1,2-bis(3,5-diphenylisothiazol-4-yl)disulfane (2) 

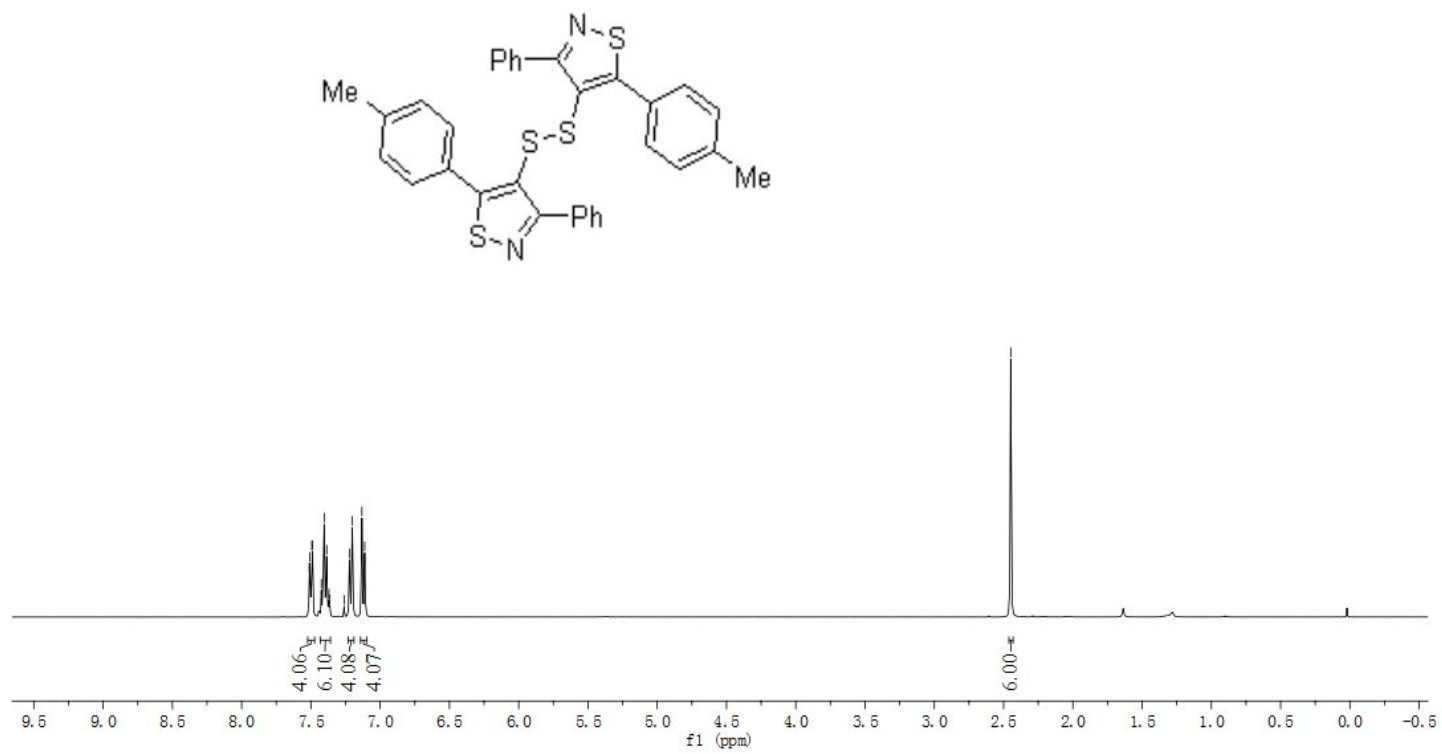

${ }^{1} \mathrm{H}$ NMR $400 \mathrm{MHz}$, in $\mathrm{CDCl}_{3}$ : 1,2-bis(3-phenyl-5-(p-tolyl)isothiazol-4-yl)disulfane

(3)

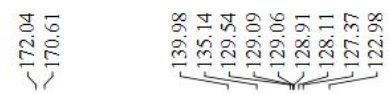

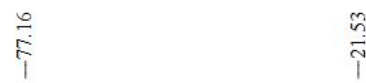
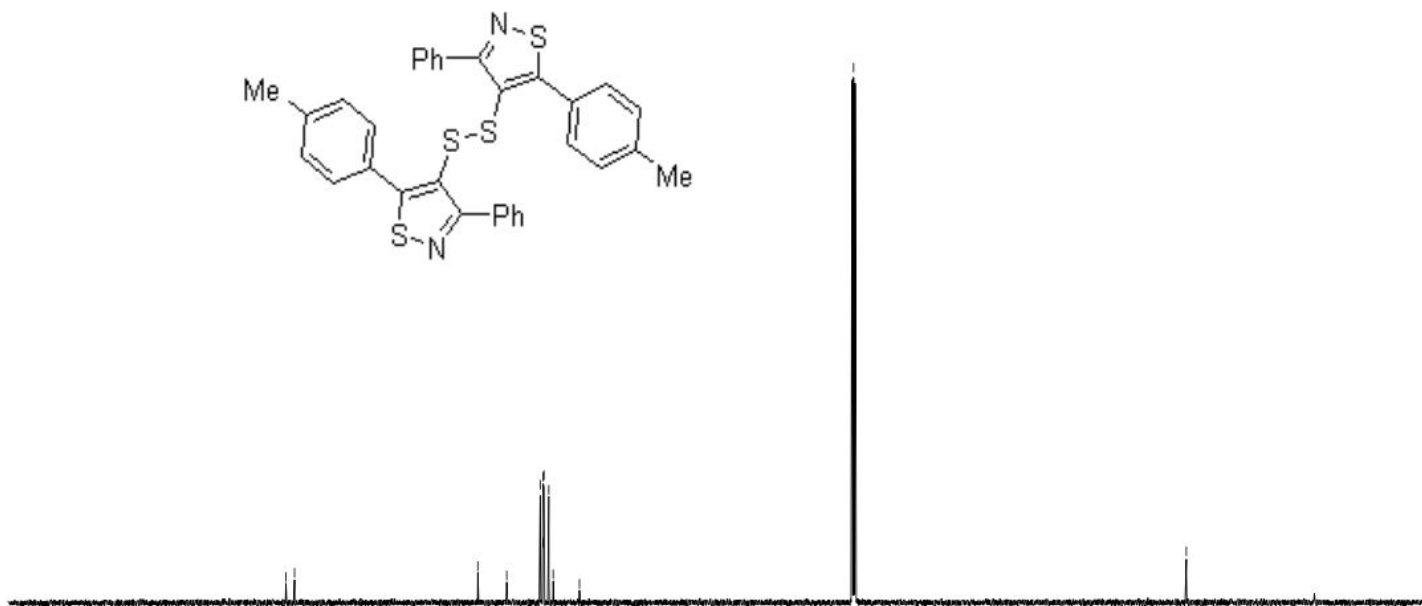

$\begin{array}{lllllllllllllllllllllll}210 & 200 & 190 & 180 & 170 & 160 & 150 & 140 & 130 & 120 & 110 & 100 & 90 & 80 & 70 & 60 & 50 & 40 & 30 & 20 & 10 & 0 & -10\end{array}$

${ }^{13} \mathrm{C}$ NMR $125 \mathrm{MHz}$, in $\mathrm{CDCl}_{3}$ :

1,2-bis(3-phenyl-5-(p-tolyl)isothiazol-4-yl)disulfane (3) 

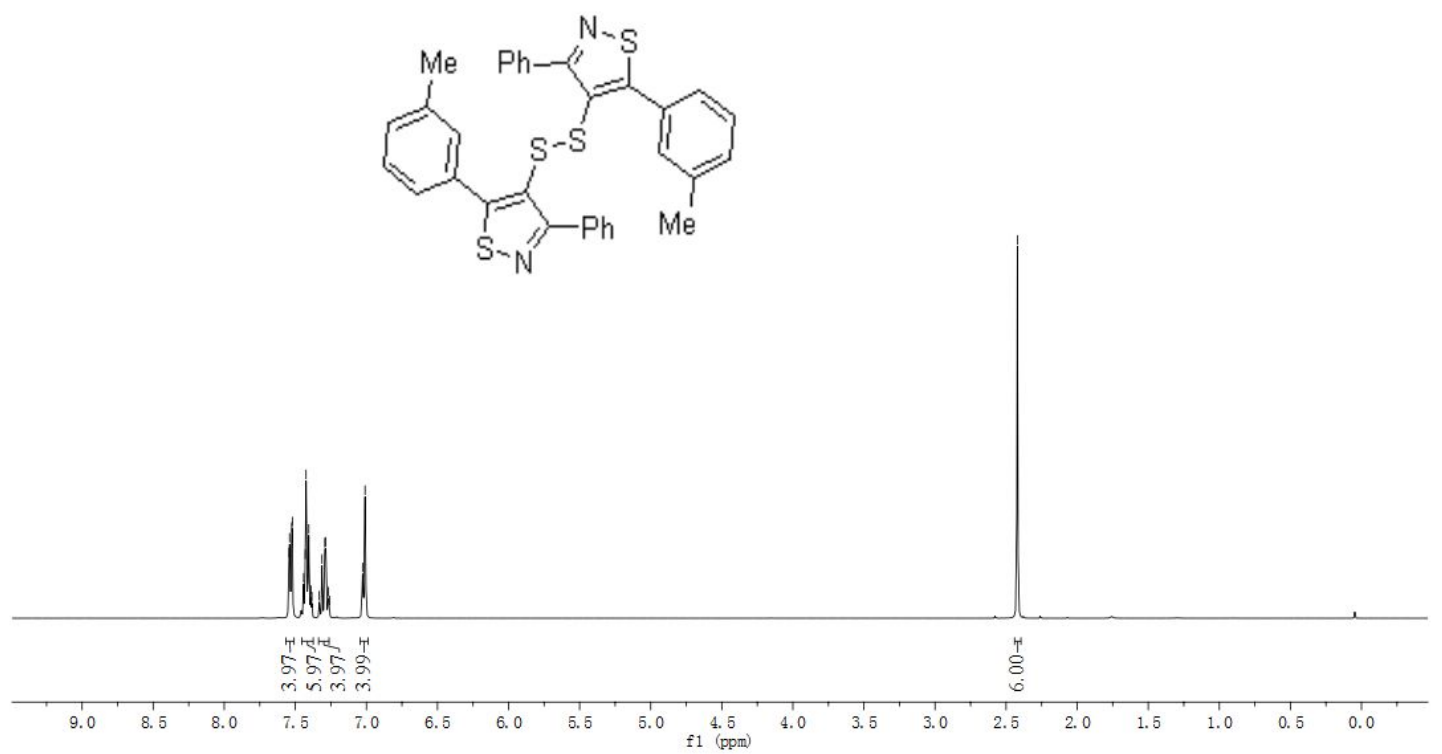

${ }^{1} \mathrm{H}$ NMR $400 \mathrm{MHz}$, in $\mathrm{CDCl}_{3}$ : 1,2-bis(3-phenyl-5-(p-tolyl)isothiazol-4-yl)disulfane

(4)
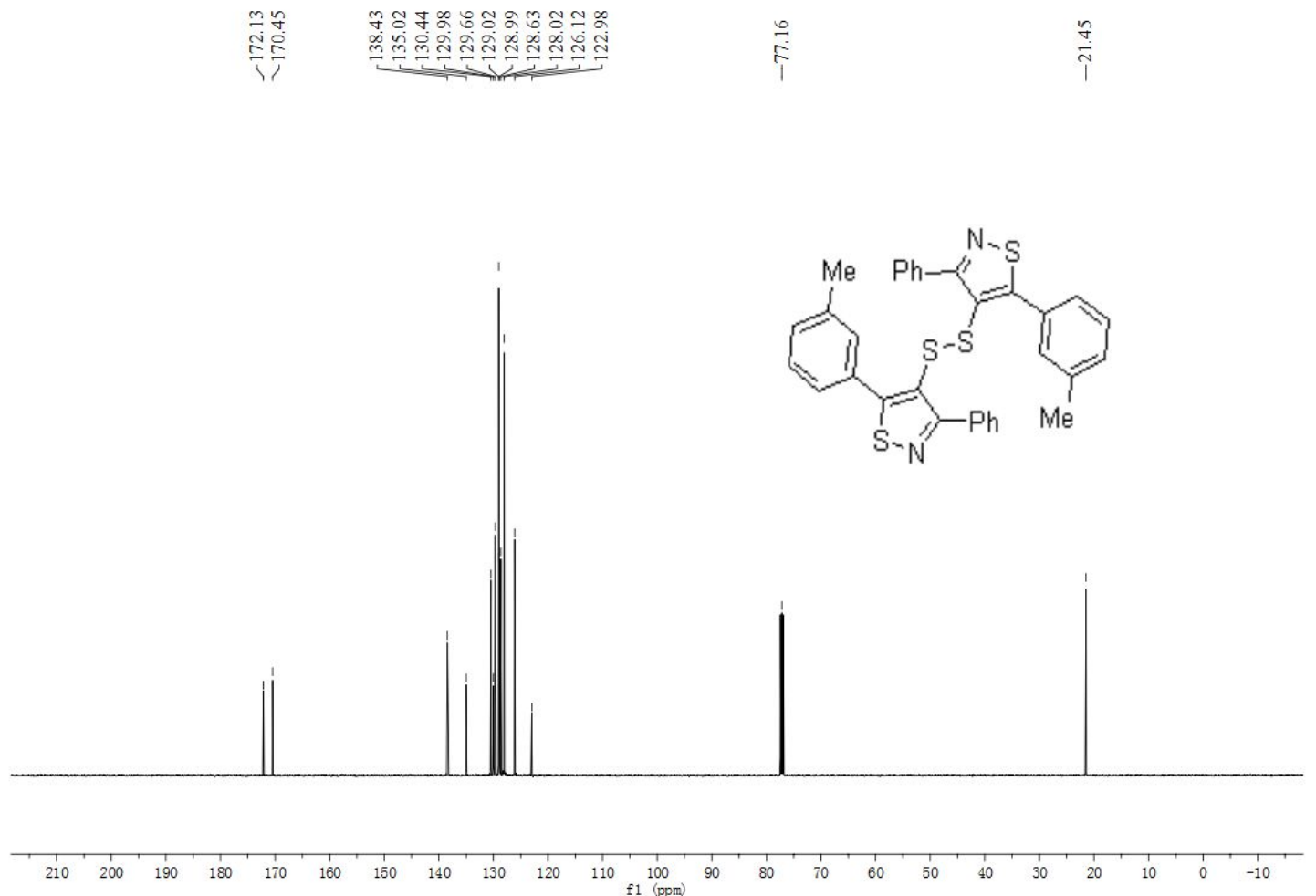

${ }^{13} \mathrm{C}$ NMR $125 \mathrm{MHz}$, in $\mathrm{CDCl}_{3}$ :

1,2-bis(3-phenyl-5-(p-tolyl)isothiazol-4-yl)disulfane (4) 

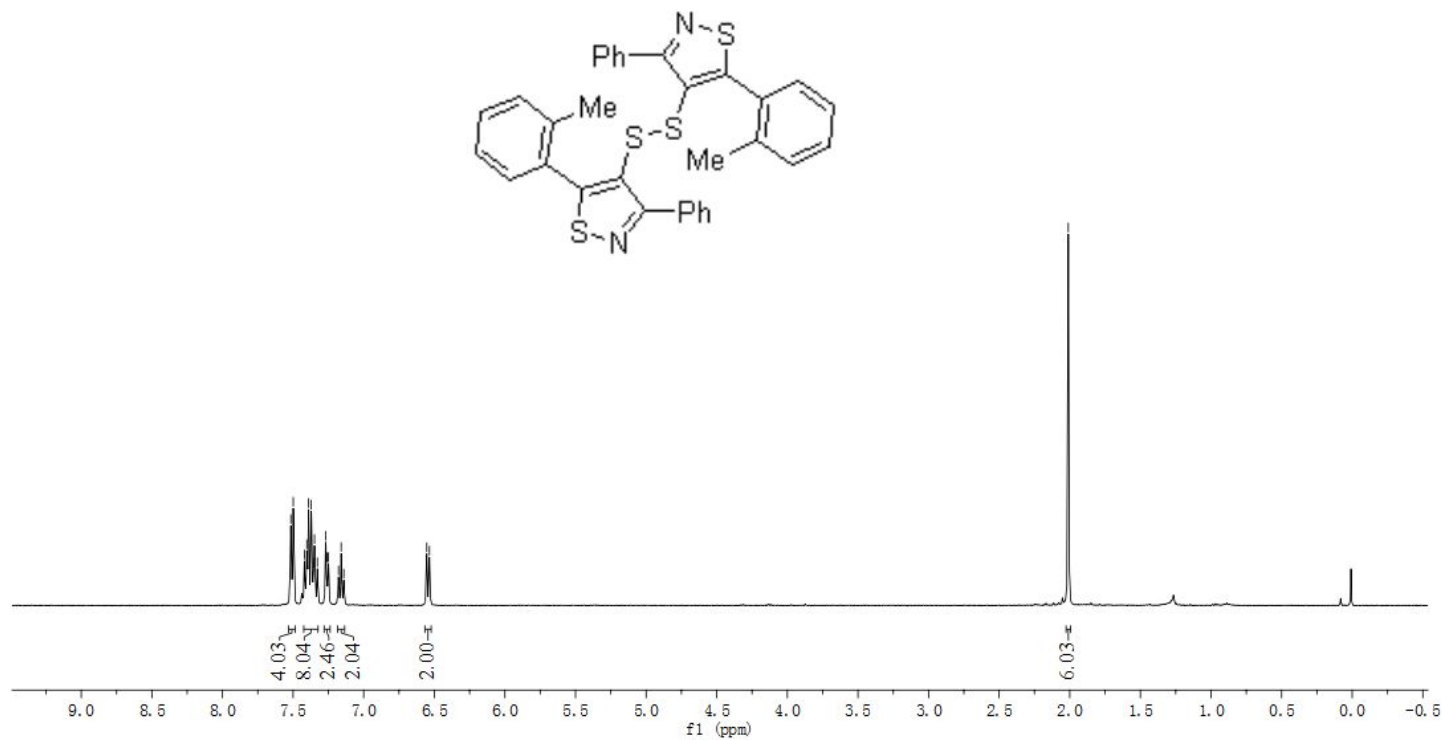

${ }^{1} \mathrm{H}$ NMR $400 \mathrm{MHz}$, in $\mathrm{CDCl}_{3}$ : 1,2-bis(3-phenyl-5-(o-tolyl)isothiazol-4-yl)disulfane

(5)

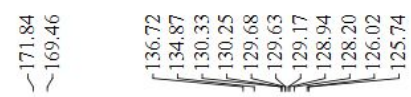

$\frac{1}{1} \sqrt{\circ}$<smiles>Cc1ccccc1-c1snc(-c2ccccc2)c1SSc1c(-c2ccccc2)nsc1-c1ccccc1</smiles>

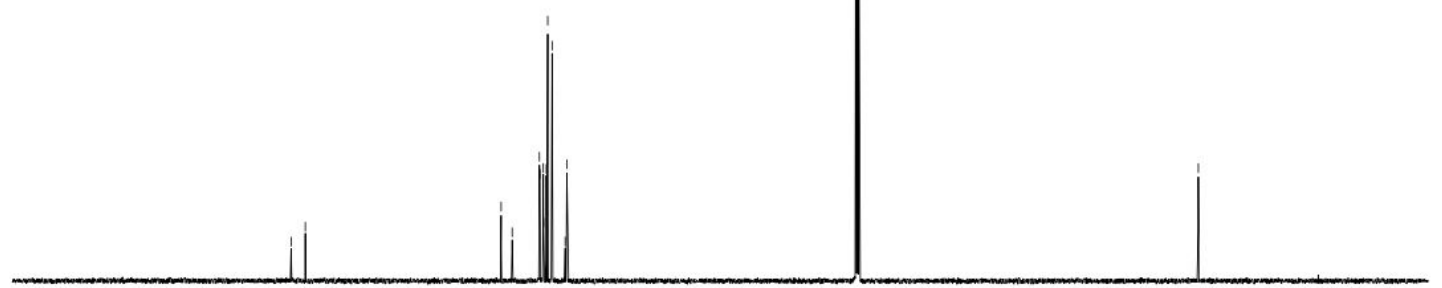

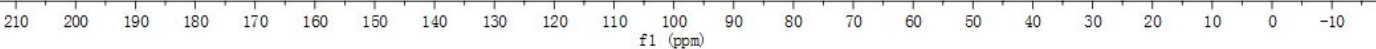

${ }^{13} \mathrm{C}$ NMR $125 \mathrm{MHz}$, in $\mathrm{CDCl}_{3}$ :

1,2-bis(3-phenyl-5-(o-tolyl)isothiazol-4-yl)disulfane (5) 

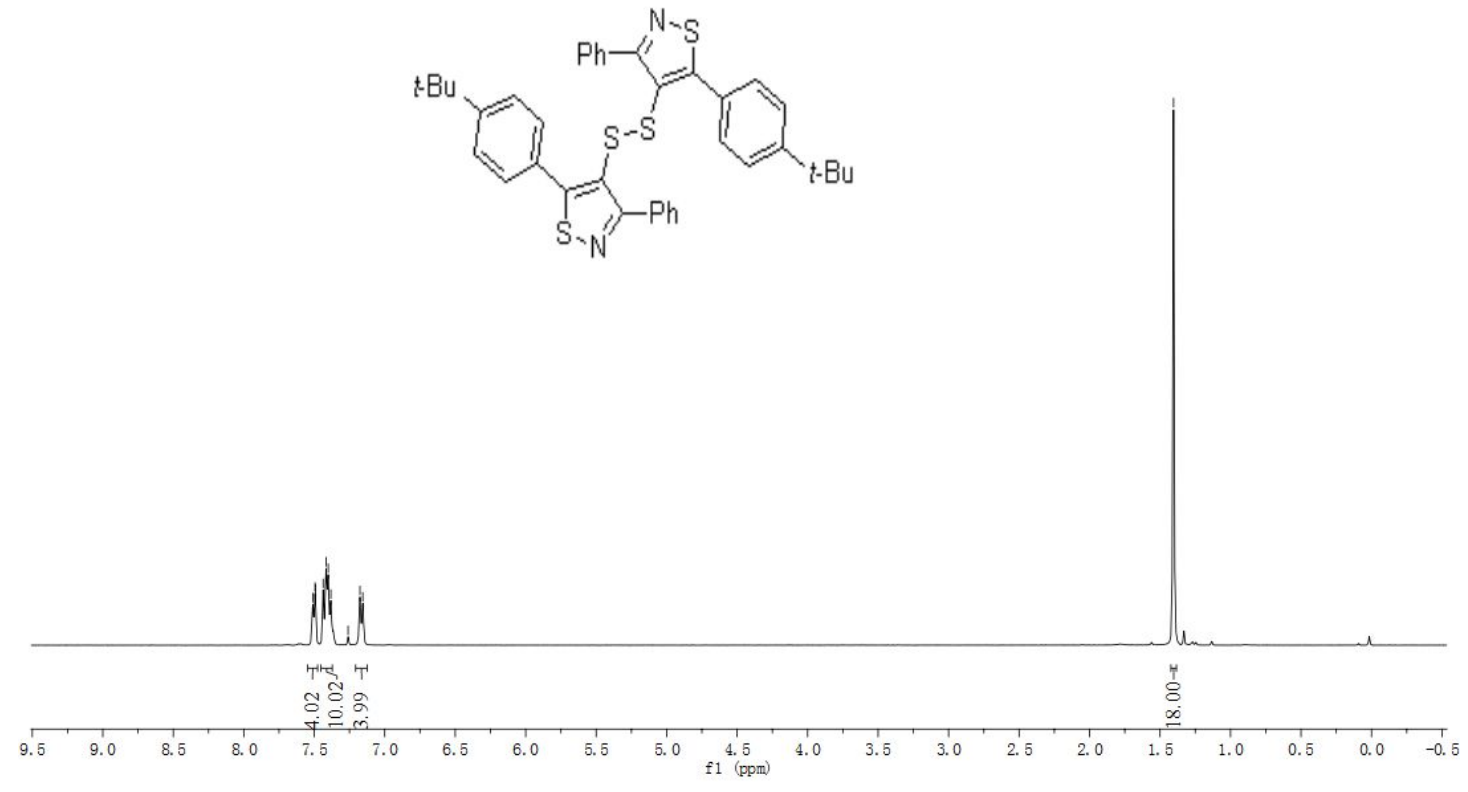

${ }^{1} \mathrm{H}$ NMR $400 \mathrm{MHz}$, in $\mathrm{CDCl}_{3}$ :

1,2-bis(5-(4-(tert-butyl)phenyl)-3-phenylisothiazol-4-yl)disulfane (6)

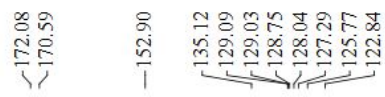
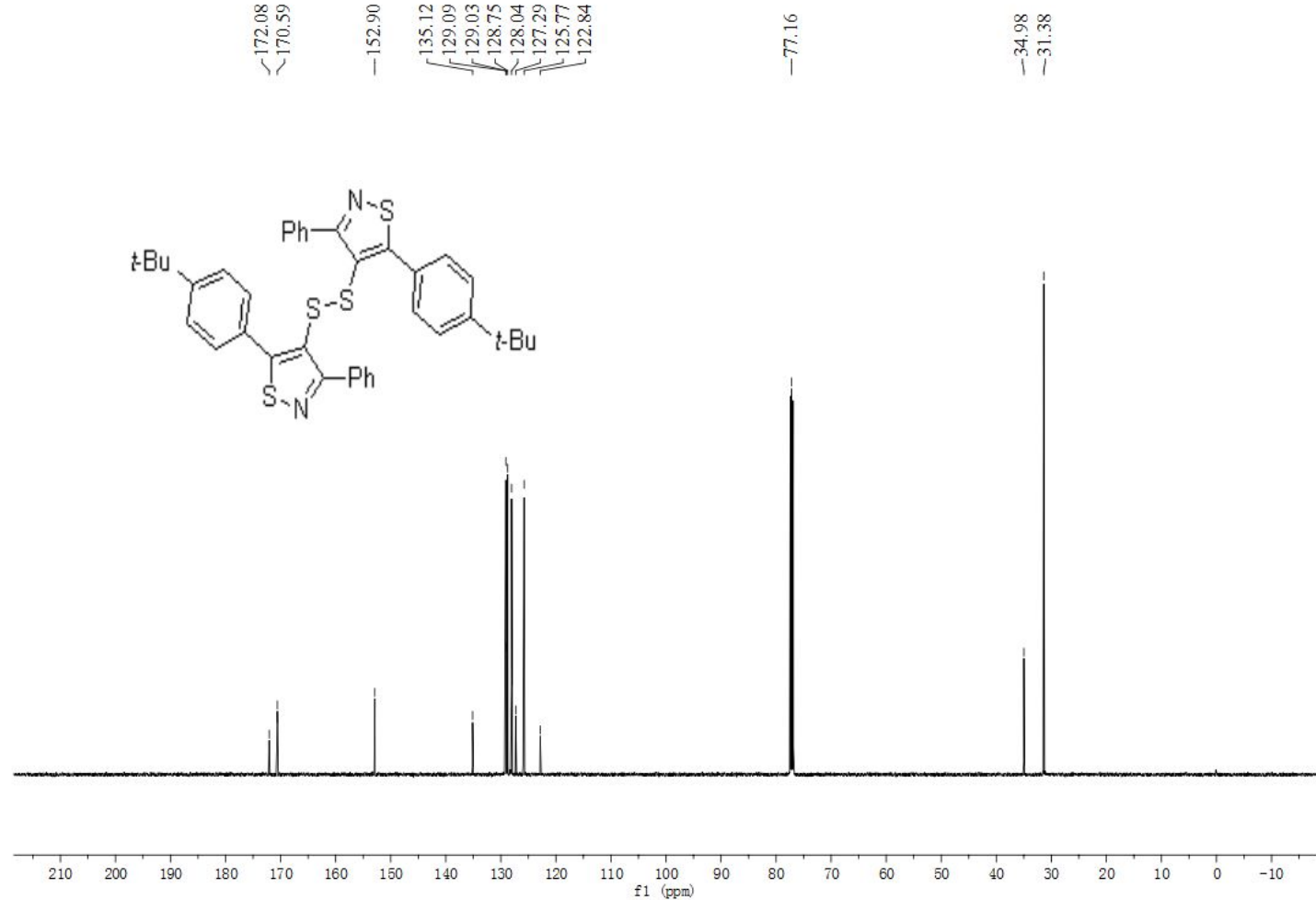

${ }^{13} \mathrm{C}$ NMR $125 \mathrm{MHz}$, in $\mathrm{CDCl}_{3}$ :

1,2-bis(5-(4-(tert-butyl)phenyl)-3-phenylisothiazol-4-yl)disulfane (6) 

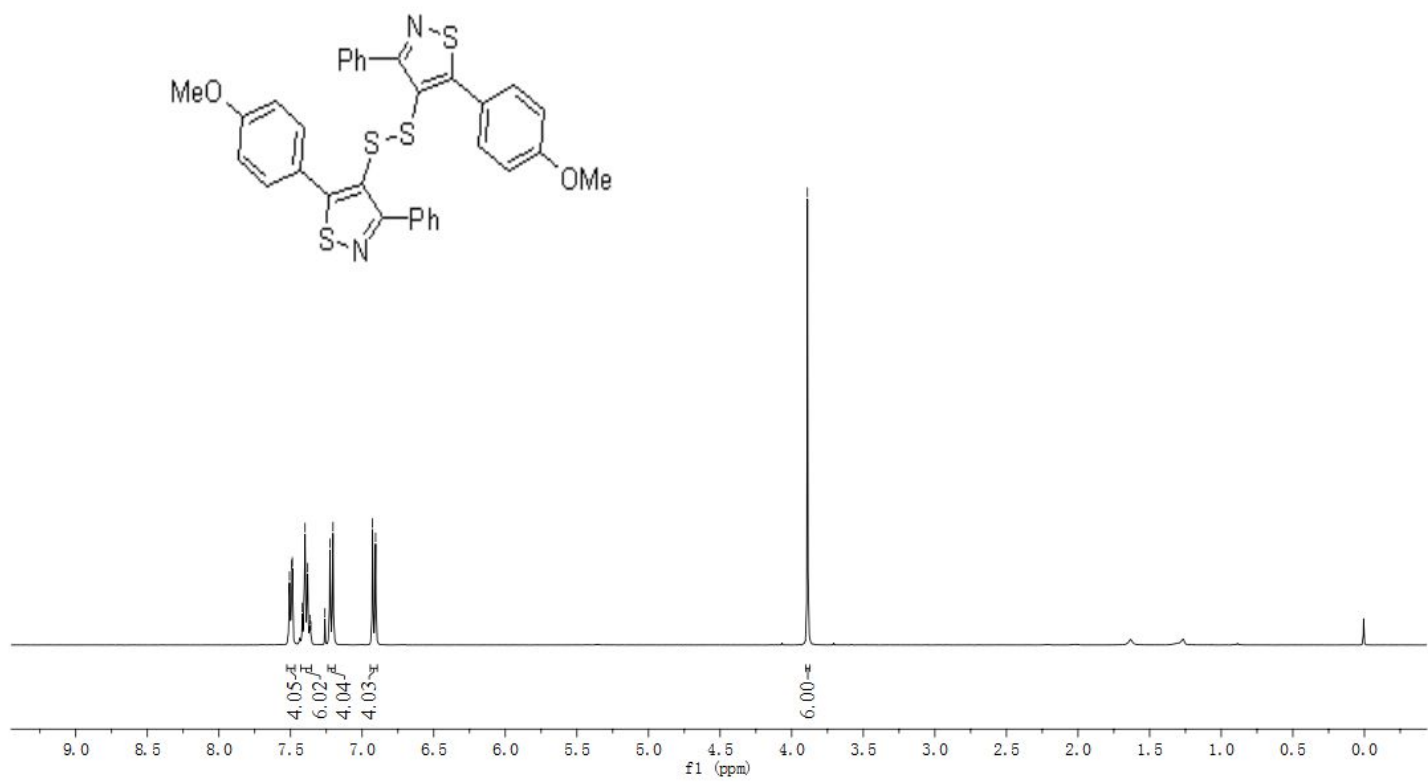

${ }^{1} \mathrm{H}$ NMR $400 \mathrm{MHz}$, in $\mathrm{CDCl}_{3}$ :

1,2-bis(5-(4-methoxyphenyl)-3-phenylisothiazol-4-yl)disulfane (7)

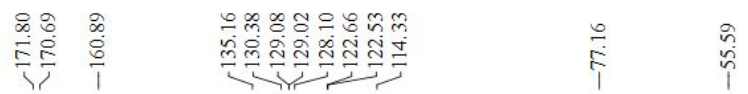
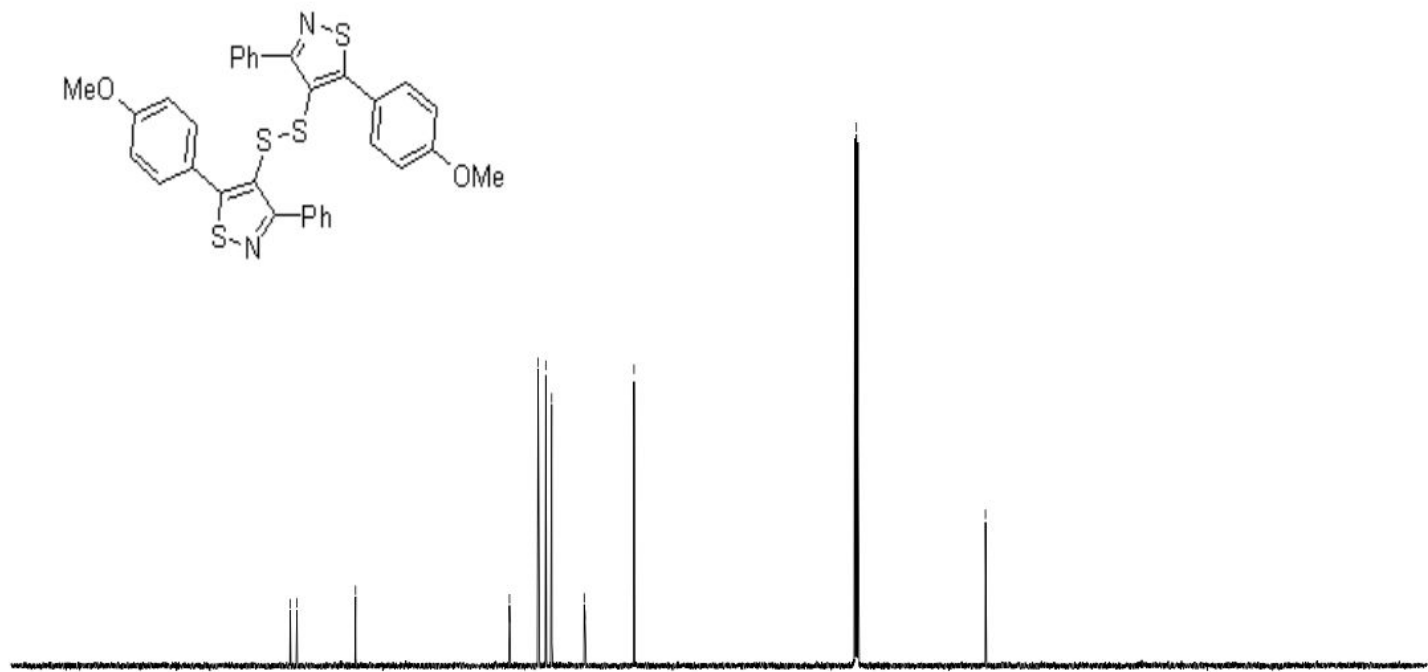

$210 \quad 200 \quad 190 \quad 180 \quad 170 \quad 160 \quad 150$

${ }^{13} \mathrm{C}$ NMR $125 \mathrm{MHz}$, in $\mathrm{CDCl}_{3}$ :

1,2-bis(5-(4-methoxyphenyl)-3-phenylisothiazol-4-yl)disulfane (7) 


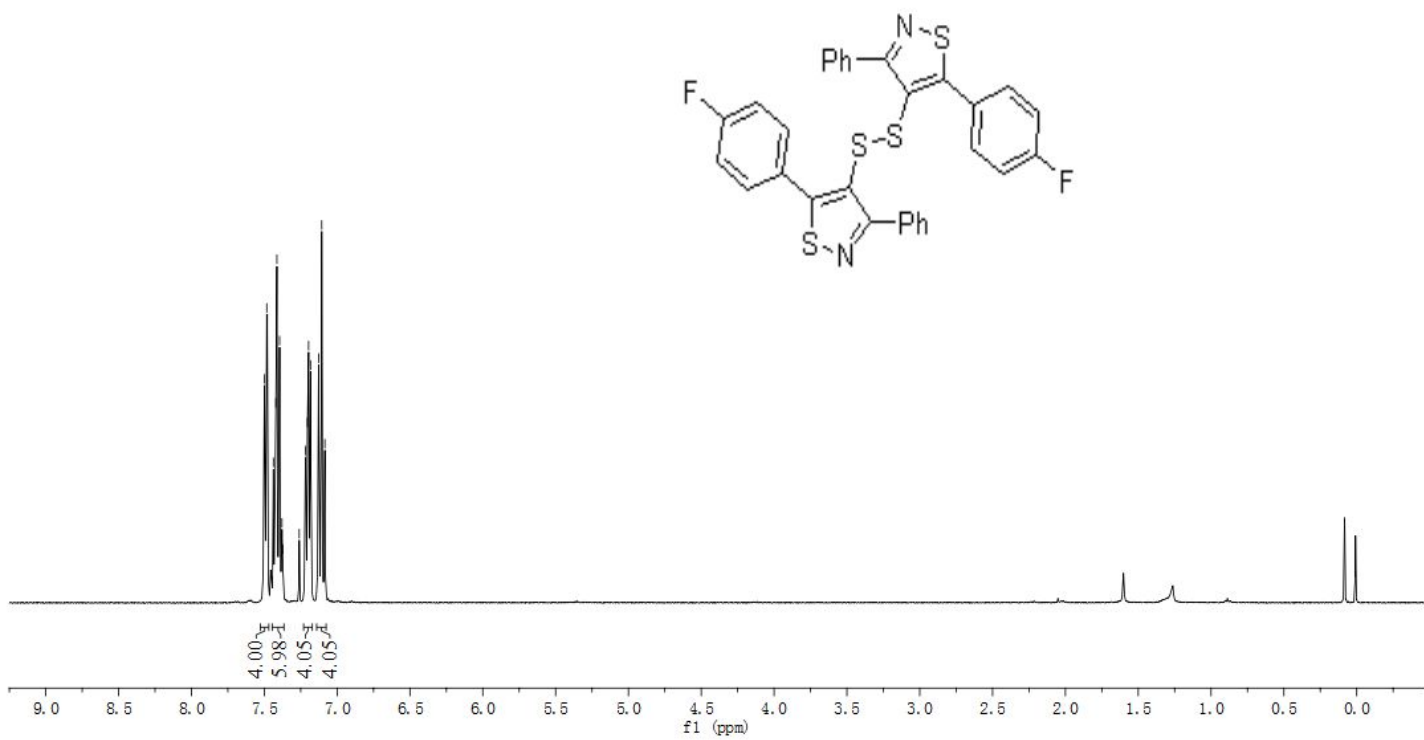

${ }^{1} \mathrm{H}$ NMR $400 \mathrm{MHz}$, in $\mathrm{CDCl}_{3}$ :

1,2-bis(5-(4-fluorophenyl)-3-phenylisothiazol-4-yl)disulfane (8)

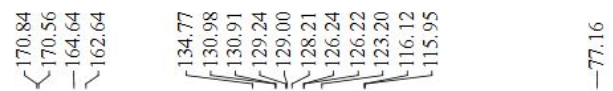

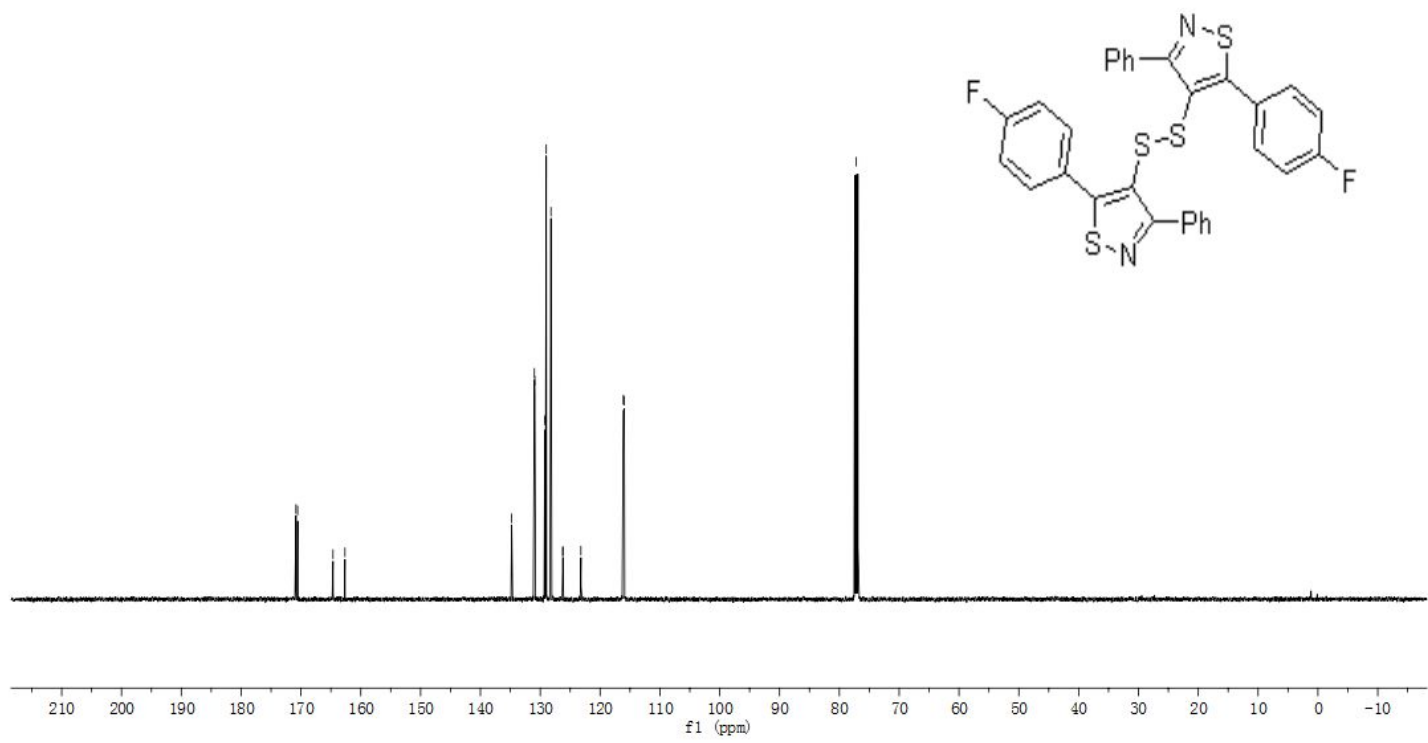

${ }^{13} \mathrm{C}$ NMR $125 \mathrm{MHz}$, in $\mathrm{CDCl}_{3}$ :

1,2-bis(5-(4-fluorophenyl)-3-phenylisothiazol-4-yl)disulfane (8) 

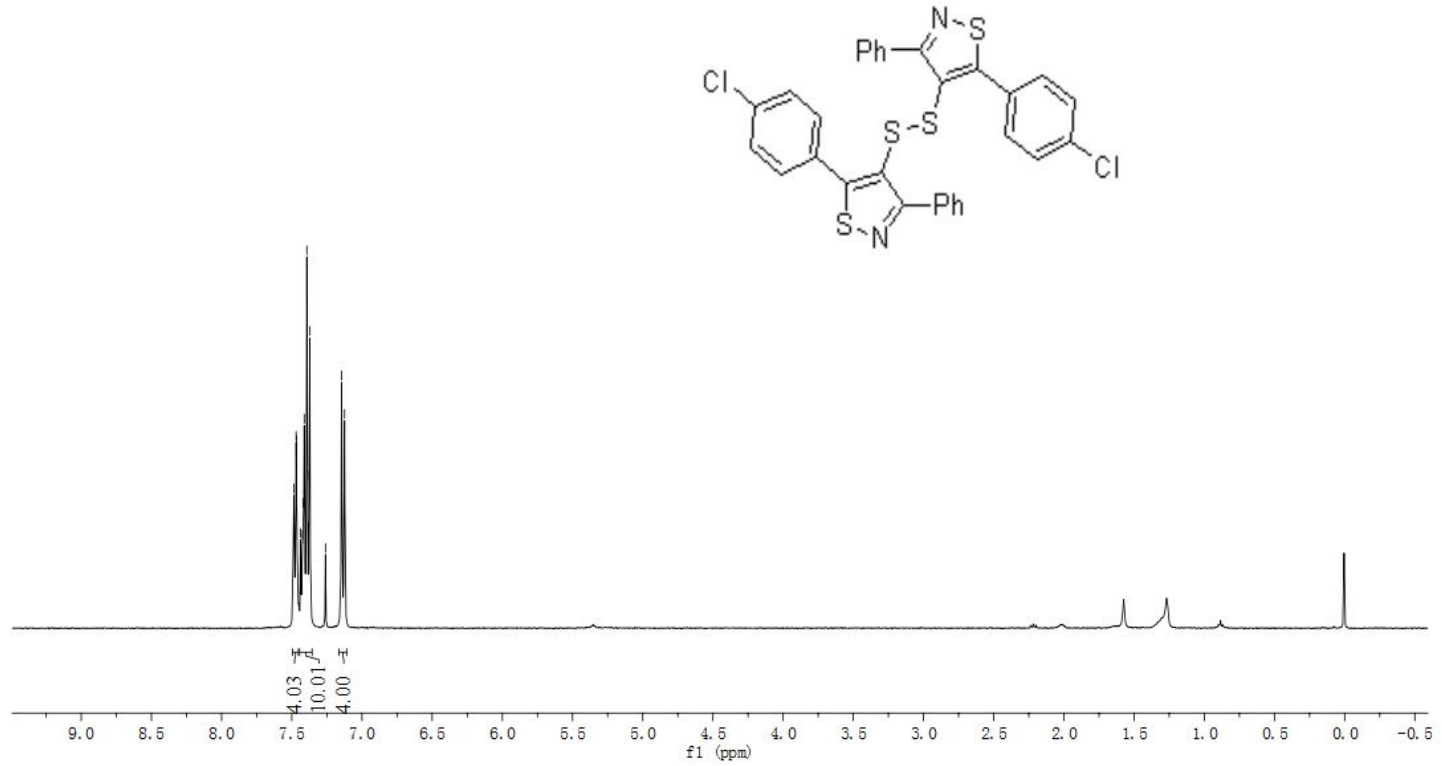

${ }^{1} \mathrm{H}$ NMR $400 \mathrm{MHz}$, in $\mathrm{CDCl}_{3}$ :

1,2-bis(5-(4-chlorophenyl)-3-phenylisothiazol-4-yl)disulfane (9)

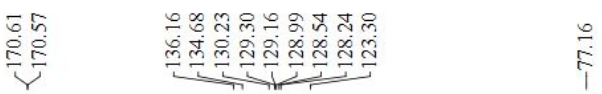

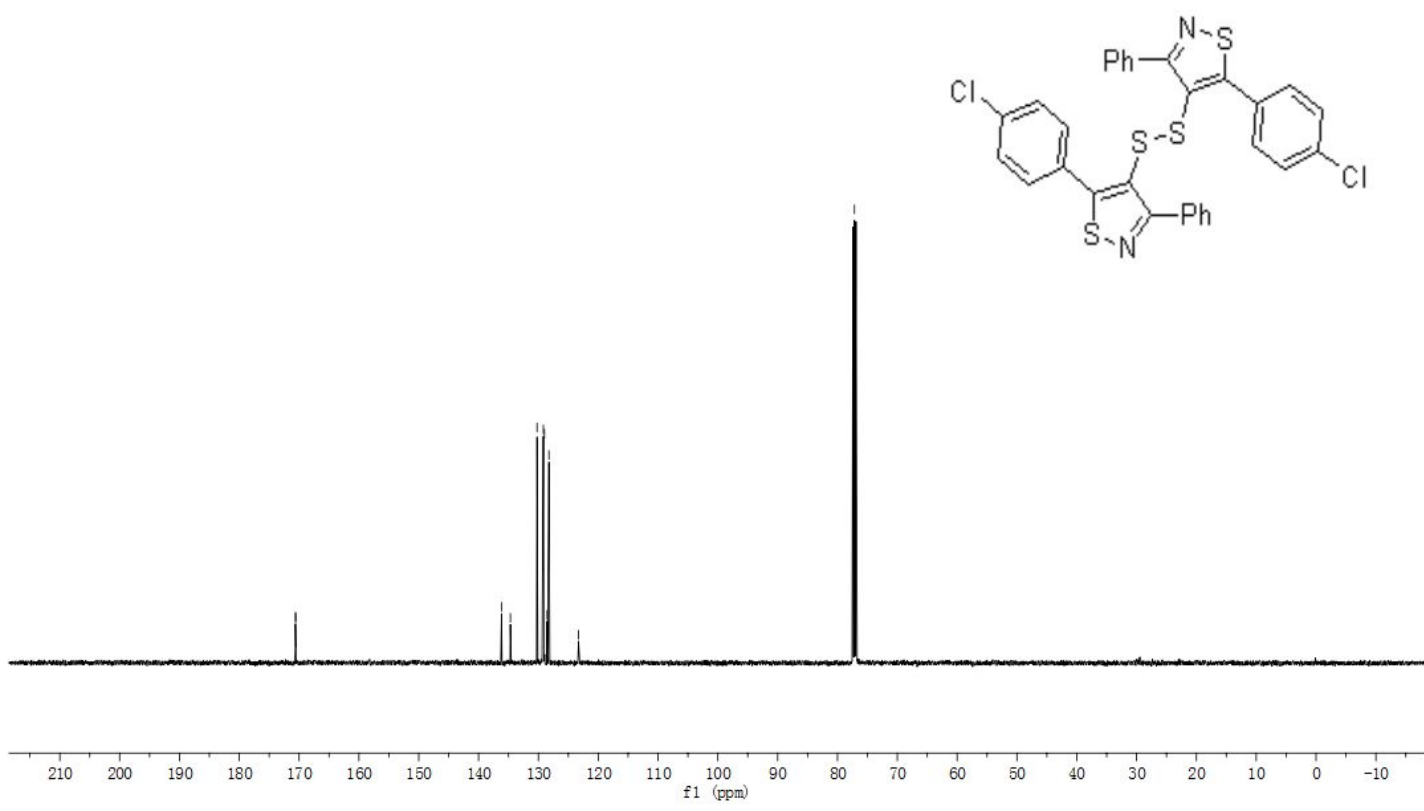

${ }^{13} \mathrm{C}$ NMR $125 \mathrm{MHz}$, in $\mathrm{CDCl}_{3}$ :

1,2-bis(5-(4-chlorophenyl)-3-phenylisothiazol-4-yl)disulfane (9) 

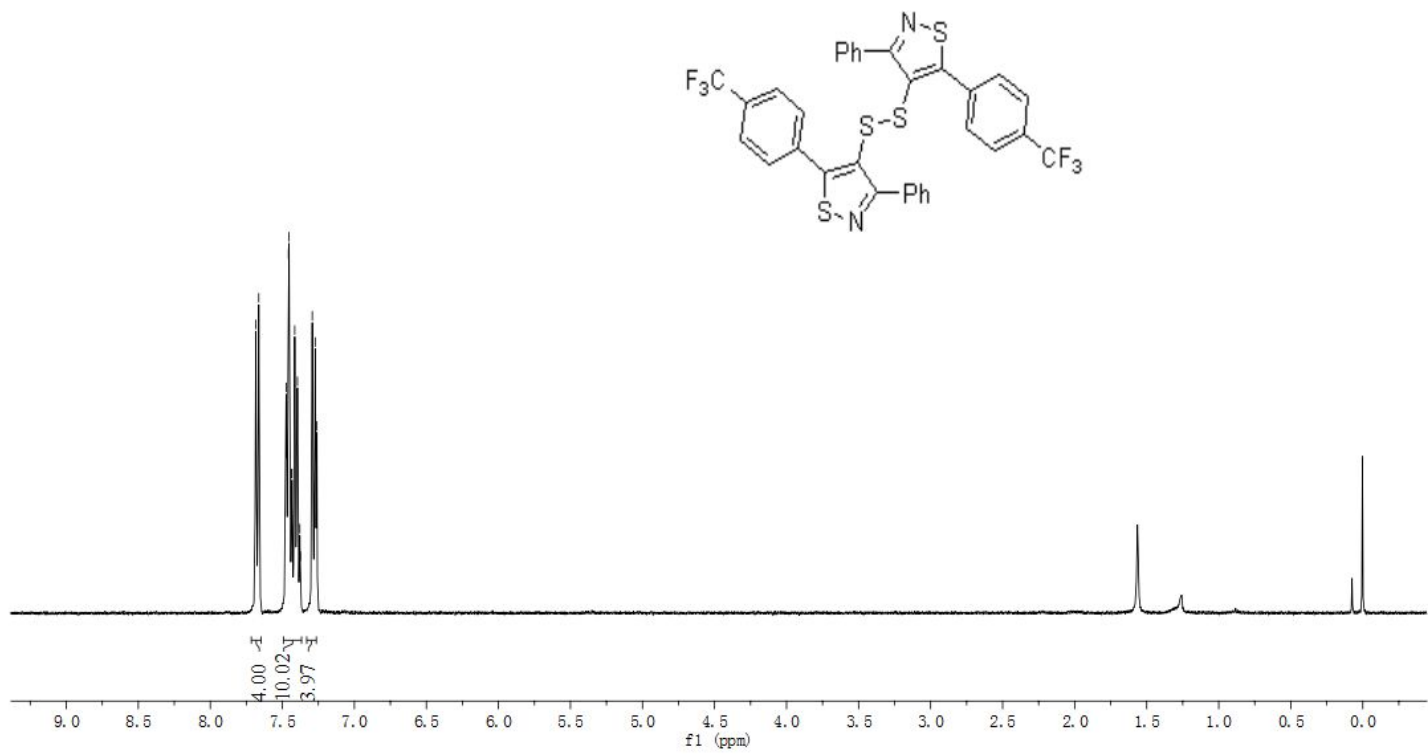

${ }^{1} \mathrm{H}$ NMR $400 \mathrm{MHz}$, in $\mathrm{CDCl}_{3}$ :

1,2-bis(3-phenyl-5-(4-(trifluoromethyl)phenyl)isothiazol-4-yl)disulfane (10)

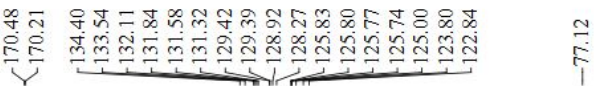

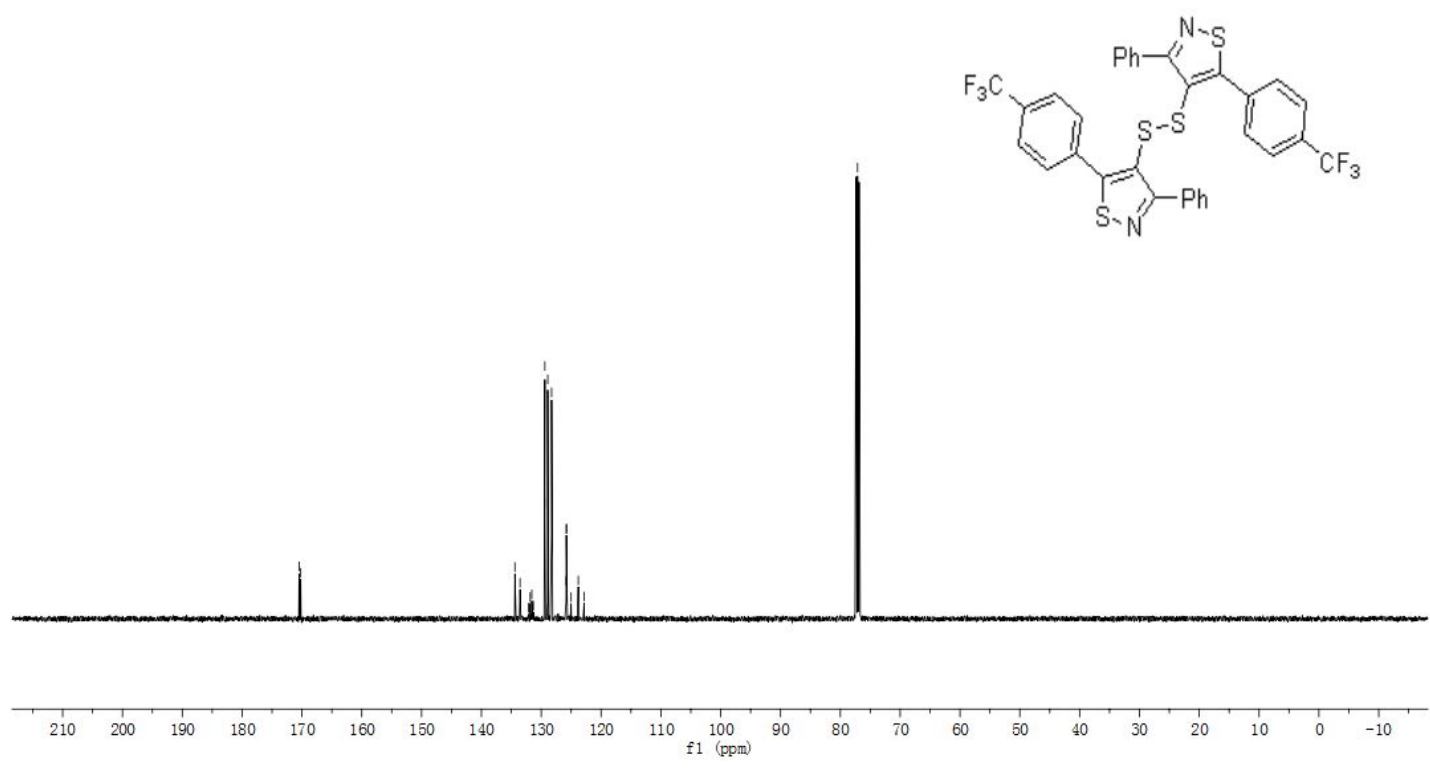

${ }^{13} \mathrm{C}$ NMR $125 \mathrm{MHz}$, in $\mathrm{CDCl}_{3}$ :

1,2-bis(3-phenyl-5-(4-(trifluoromethyl)phenyl)isothiazol-4-yl)disulfane (10) 

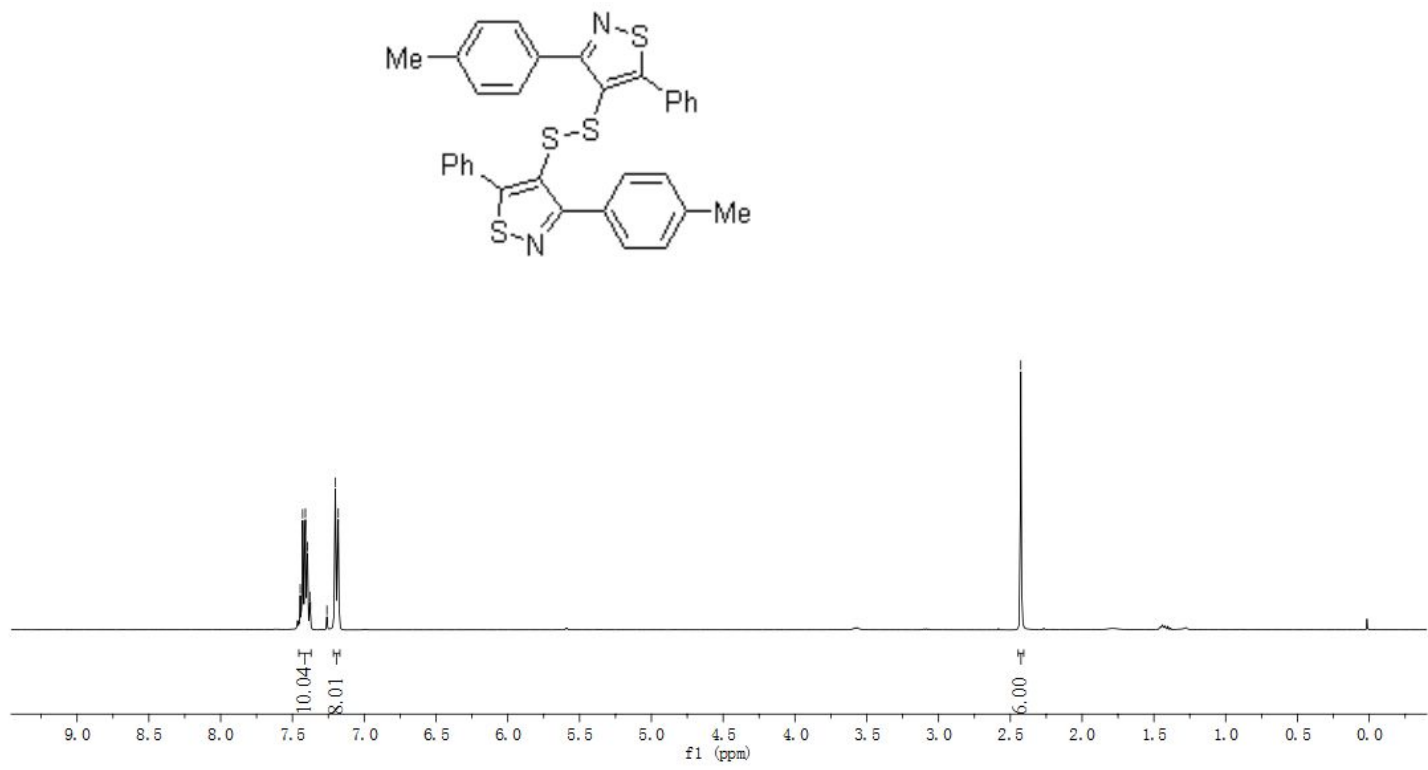

${ }^{1} \mathrm{H}$ NMR $400 \mathrm{MHz}$, in $\mathrm{CDCl}_{3}$ : 1,2-bis(5-phenyl-3-(p-tolyl)isothiazol-4-yl)disulfane

(11)

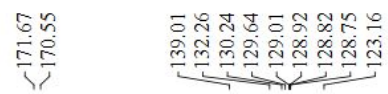

$\stackrel{n}{i} \stackrel{n}{i}$

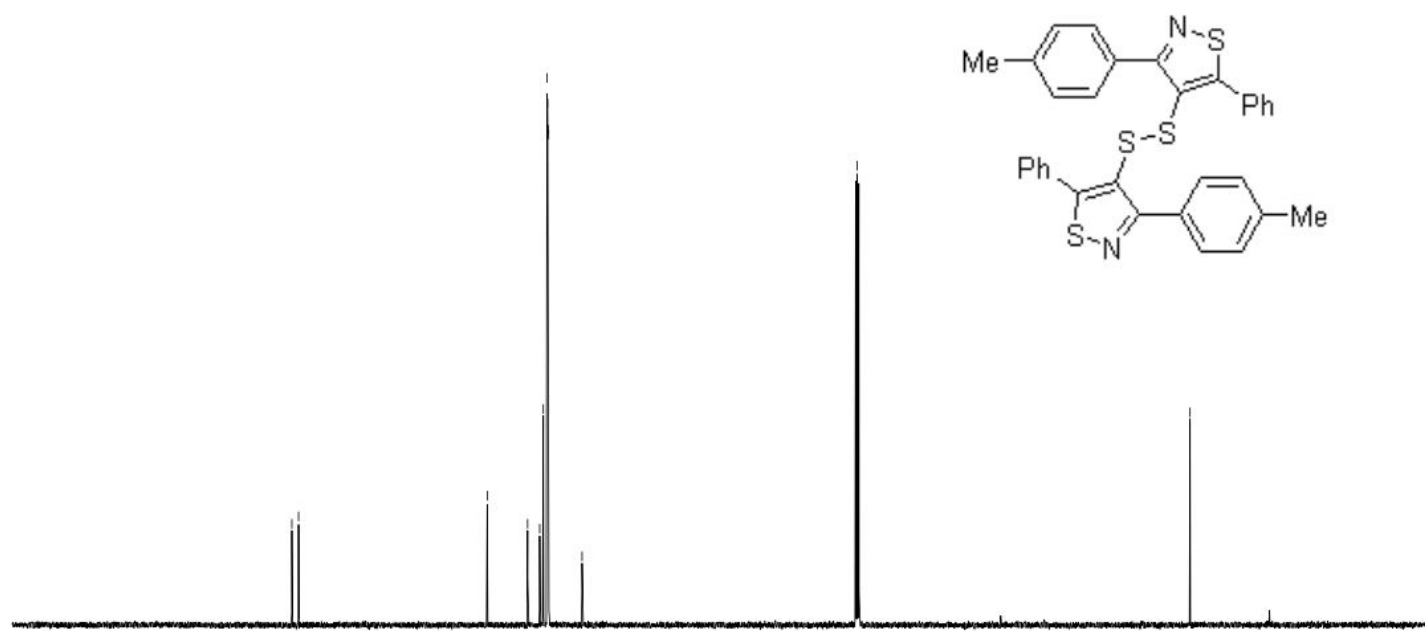

$\begin{array}{lllllllllllllllllllllllll}210 & 200 & 190 & 180 & 170 & 160 & 150 & 140 & 130 & 120 & 110 & 100 & 90 & 80 & 70 & 60 & 50 & 40 & 30 & 20 & 10 & 0 & -10\end{array}$

${ }^{13} \mathrm{C}$ NMR $125 \mathrm{MHz}$, in $\mathrm{CDCl}_{3}$ :

1,2-bis(5-phenyl-3-(p-tolyl)isothiazol-4-yl)disulfane (11) 

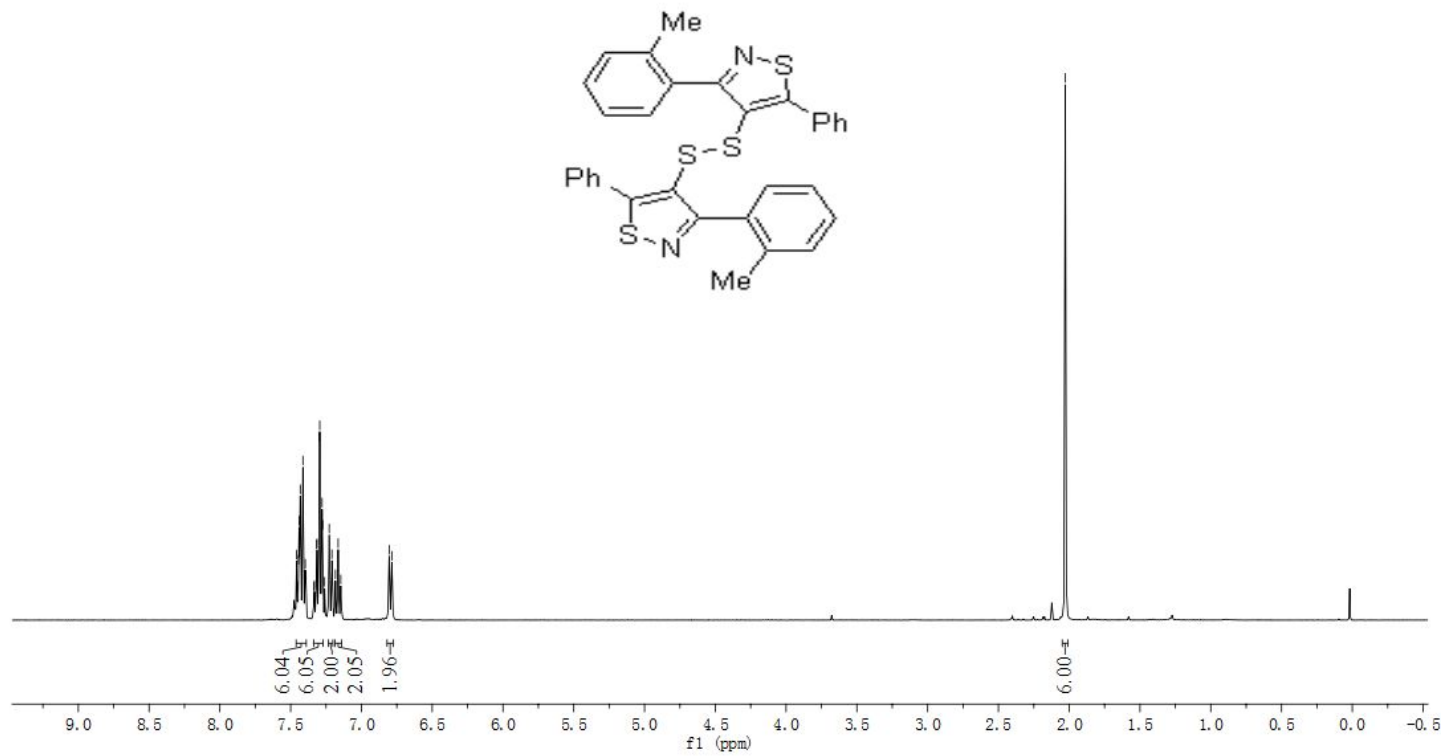

${ }^{1} \mathrm{H}$ NMR $400 \mathrm{MHz}$, in $\mathrm{CDCl}_{3}$ : 1,2-bis(5-phenyl-3-(o-tolyl)isothiazol-4-yl)disulfane
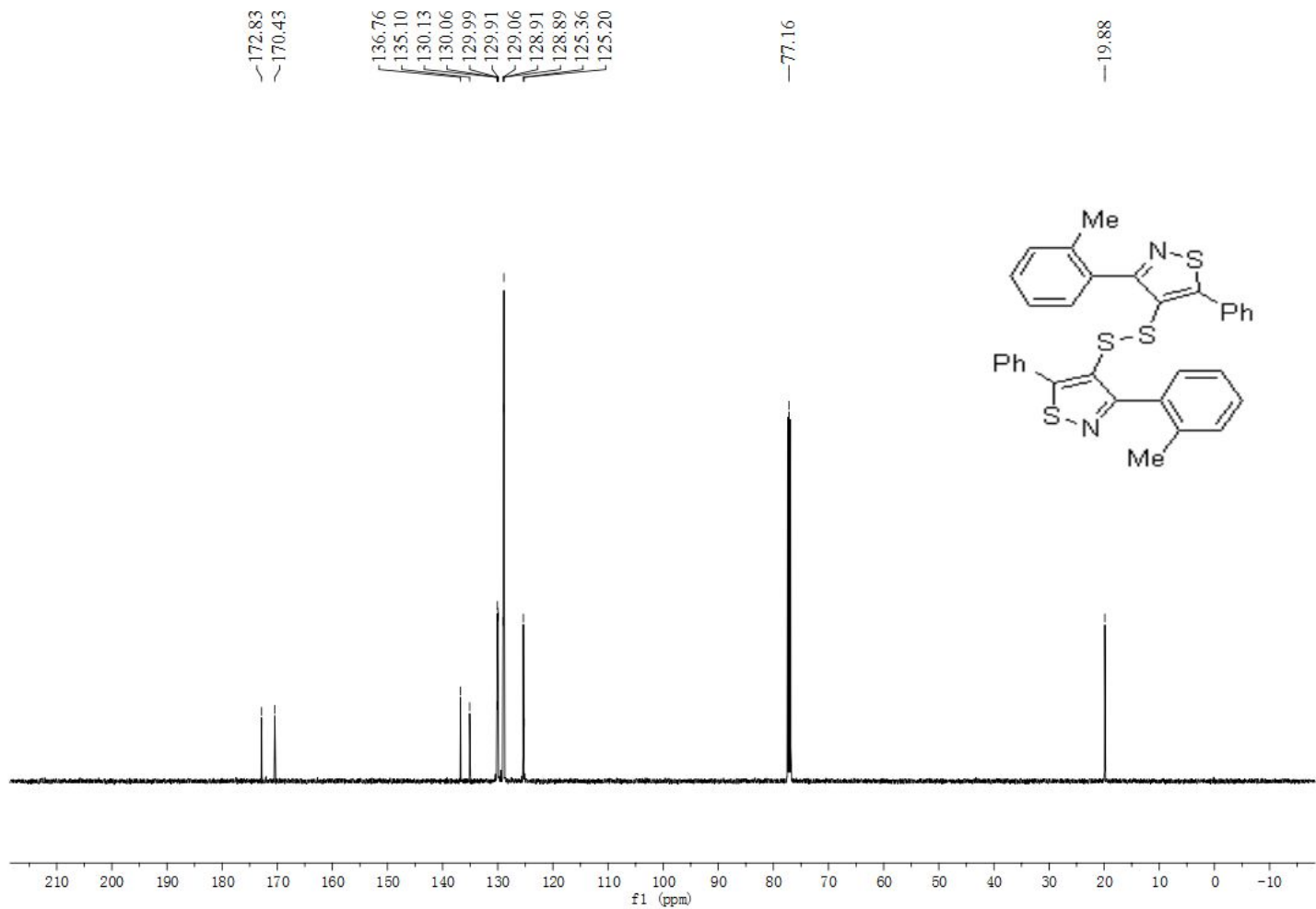

${ }^{13} \mathrm{C}$ NMR $125 \mathrm{MHz}$, in $\mathrm{CDCl}_{3}$ :

1,2-bis(5-phenyl-3-(o-tolyl)isothiazol-4-yl)disulfane (12) 

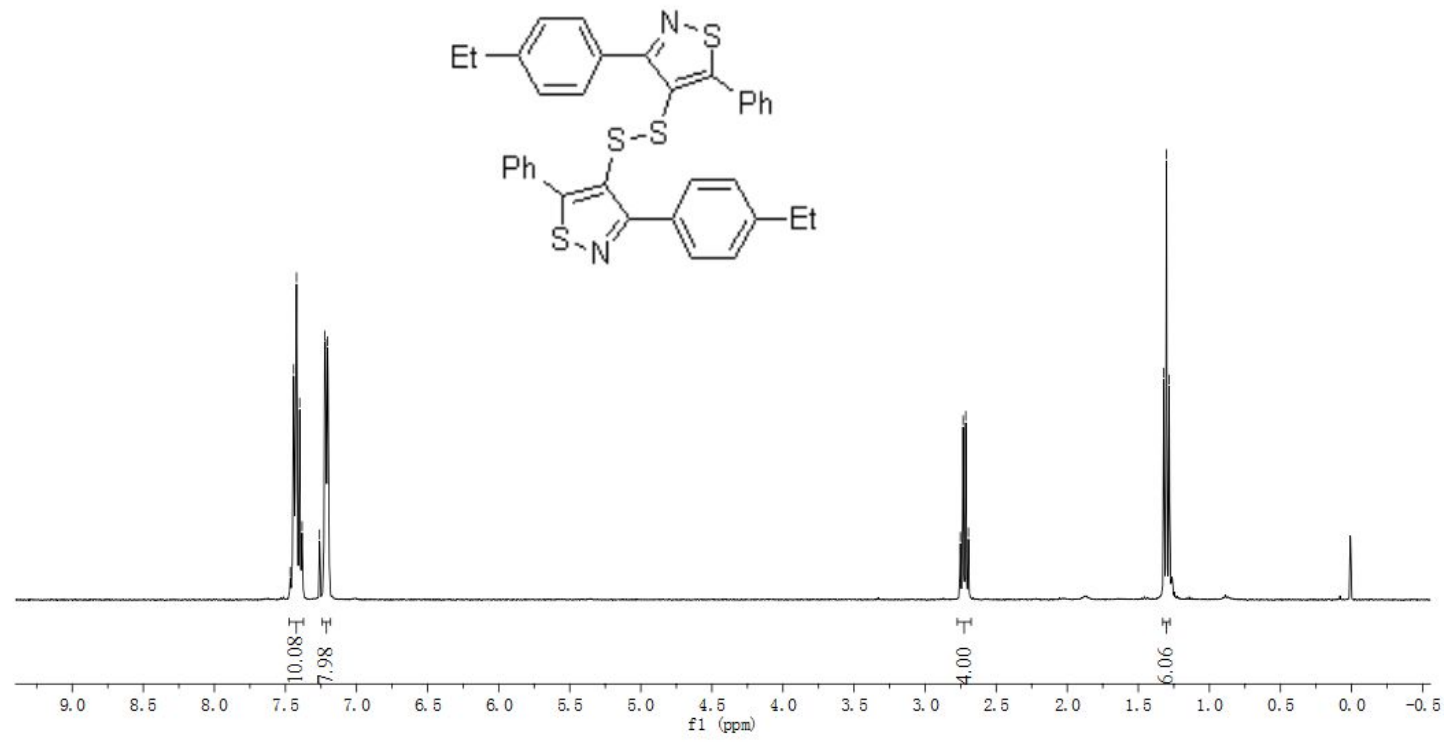

${ }^{1} \mathrm{H}$ NMR $400 \mathrm{MHz}$, in $\mathrm{CDCl}_{3}$ :

1,2-bis(3-(4-ethylphenyl)-5-phenylisothiazol-4-yl)disulfane (13)

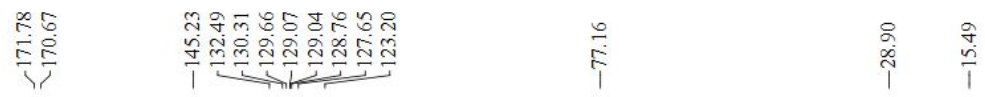

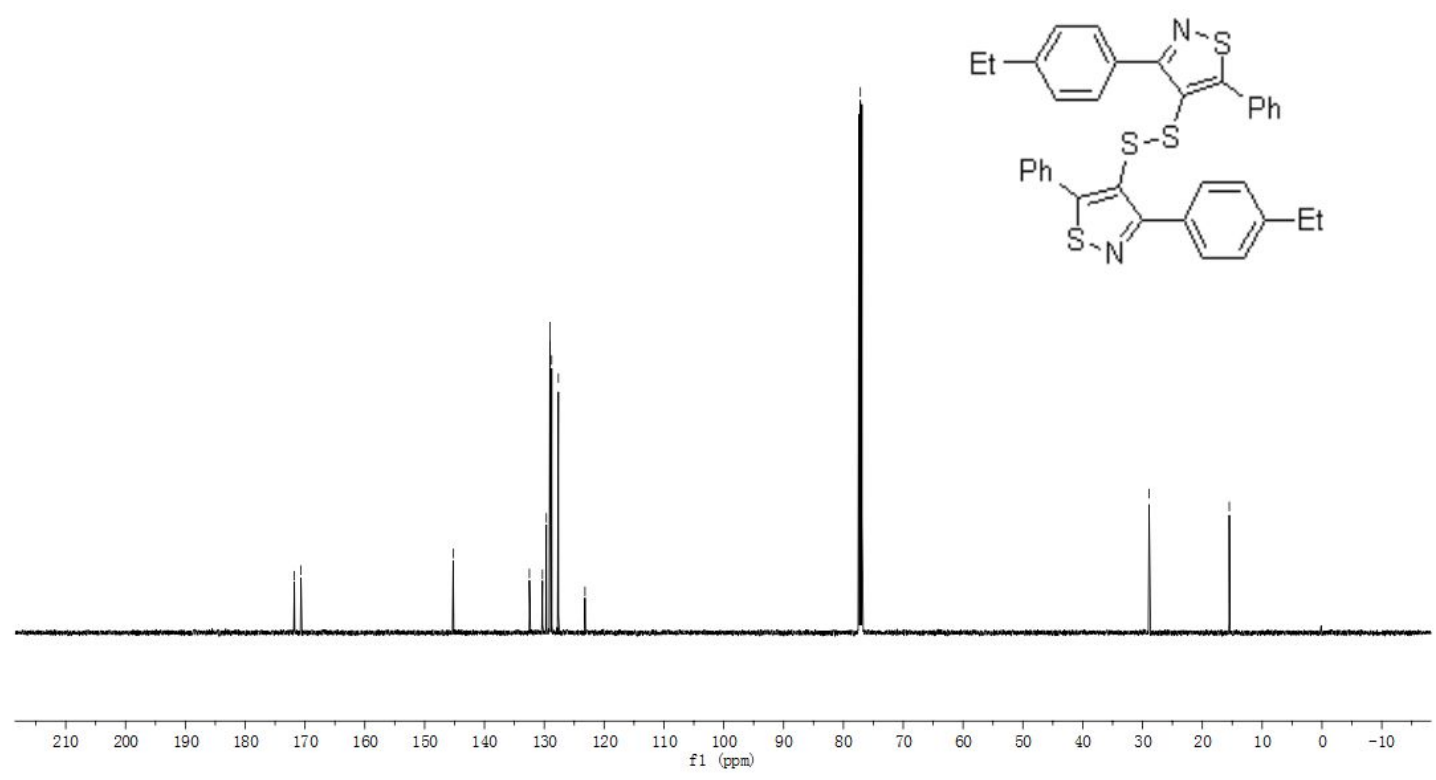

${ }^{13} \mathrm{C}$ NMR $125 \mathrm{MHz}$, in $\mathrm{CDCl}_{3}$ :

1,2-bis(3-(4-ethylphenyl)-5-phenylisothiazol-4-yl)disulfane (13) 

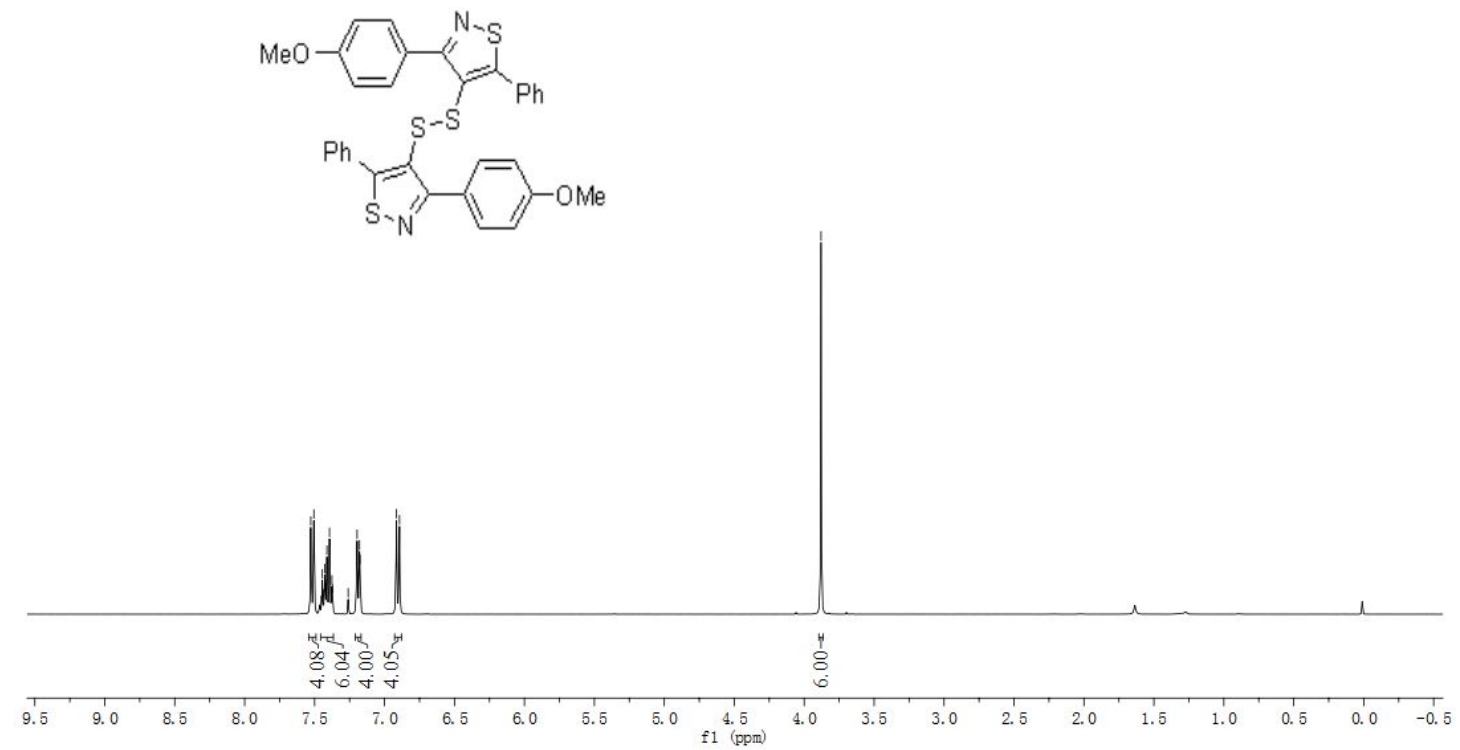

${ }^{1} \mathrm{H}$ NMR $400 \mathrm{MHz}$, in $\mathrm{CDCl}_{3}$ :

1,2-bis(3-(4-methoxyphenyl)-5-phenylisothiazol-4-yl)disulfane (14)

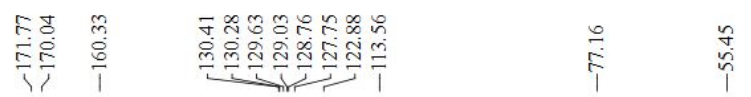

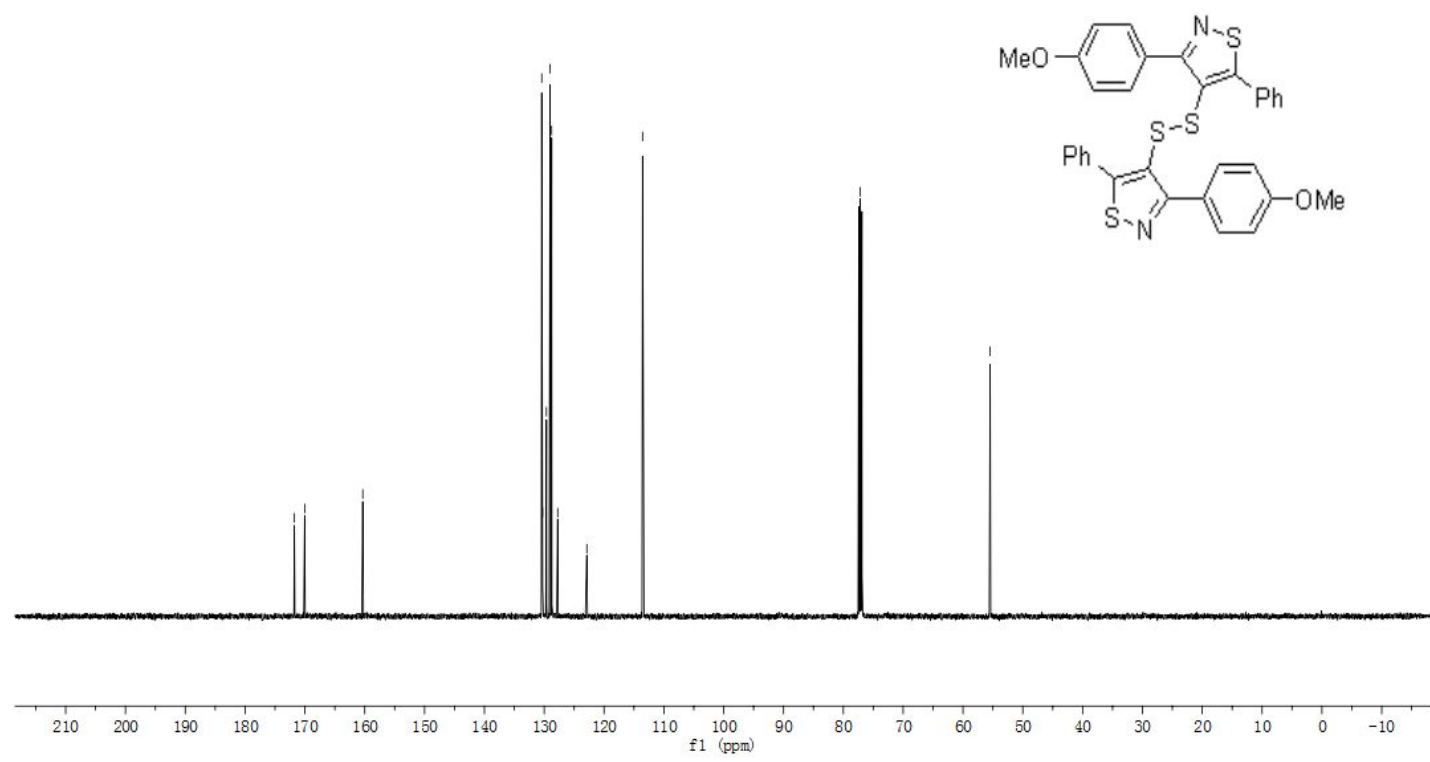

${ }^{13} \mathrm{C}$ NMR $125 \mathrm{MHz}$, in $\mathrm{CDCl}_{3}$ :

1,2-bis(3-(4-methoxyphenyl)-5-phenylisothiazol-4-yl)disulfane (14) 

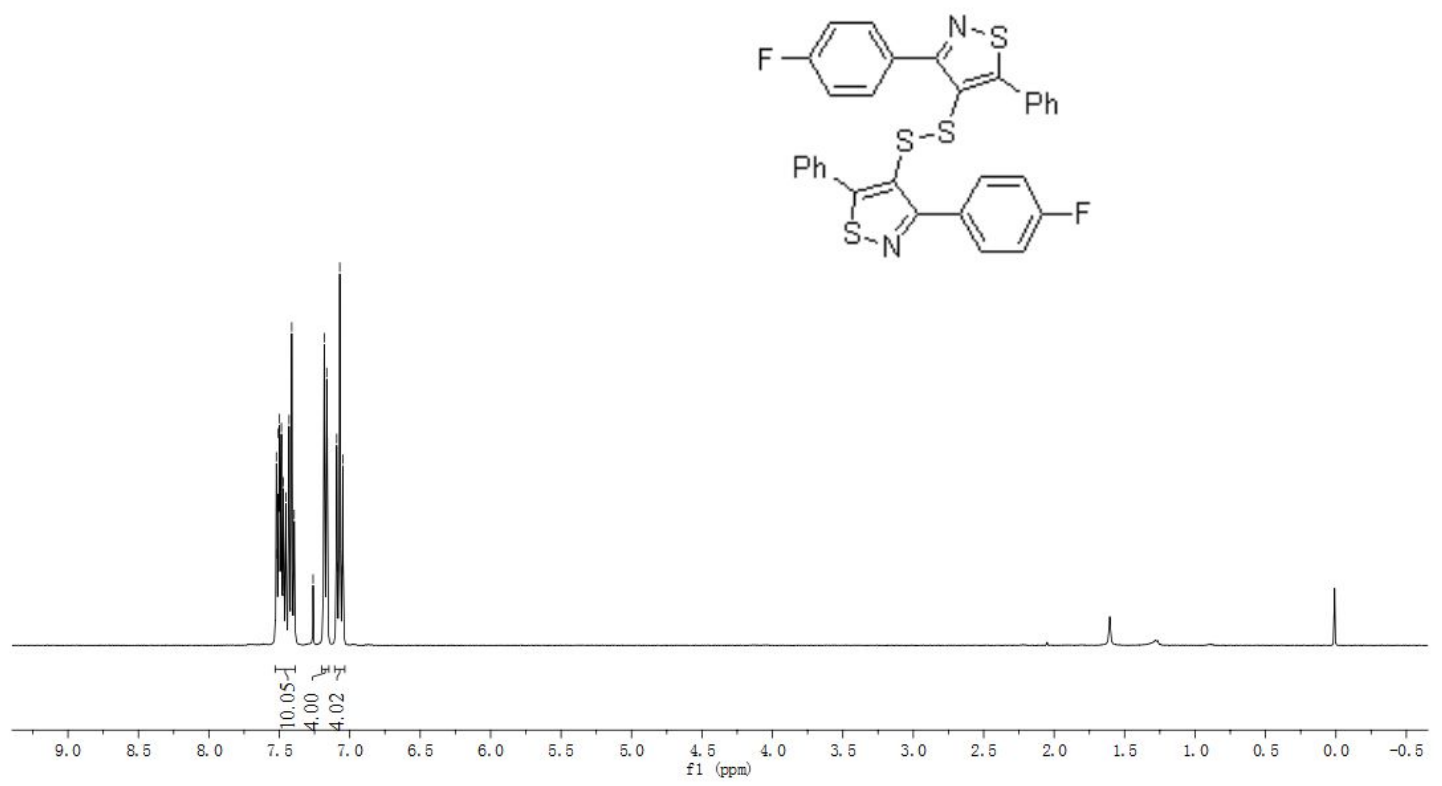

${ }^{1} \mathrm{H}$ NMR $400 \mathrm{MHz}$, in $\mathrm{CDCl}_{3}$ :

1,2-bis(3-(4-fluorophenyl)-5-phenylisothiazol-4-yl)disulfane (15)

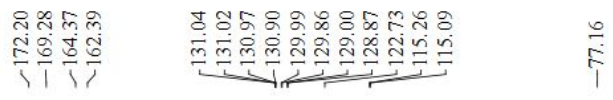

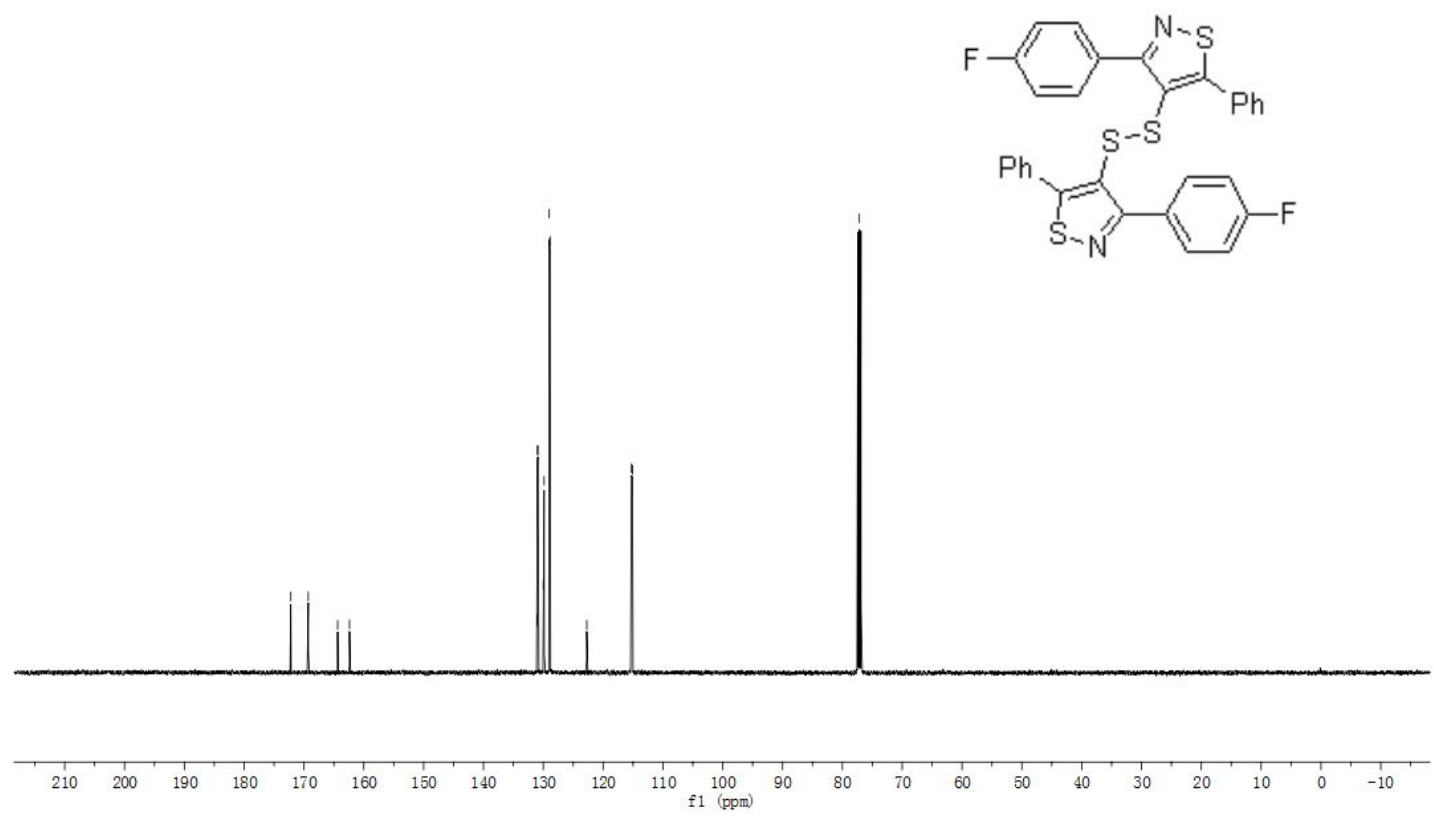

${ }^{13} \mathrm{C}$ NMR $125 \mathrm{MHz}$, in $\mathrm{CDCl}_{3}$ :

1,2-bis(3-(4-fluorophenyl)-5-phenylisothiazol-4-yl)disulfane (15) 


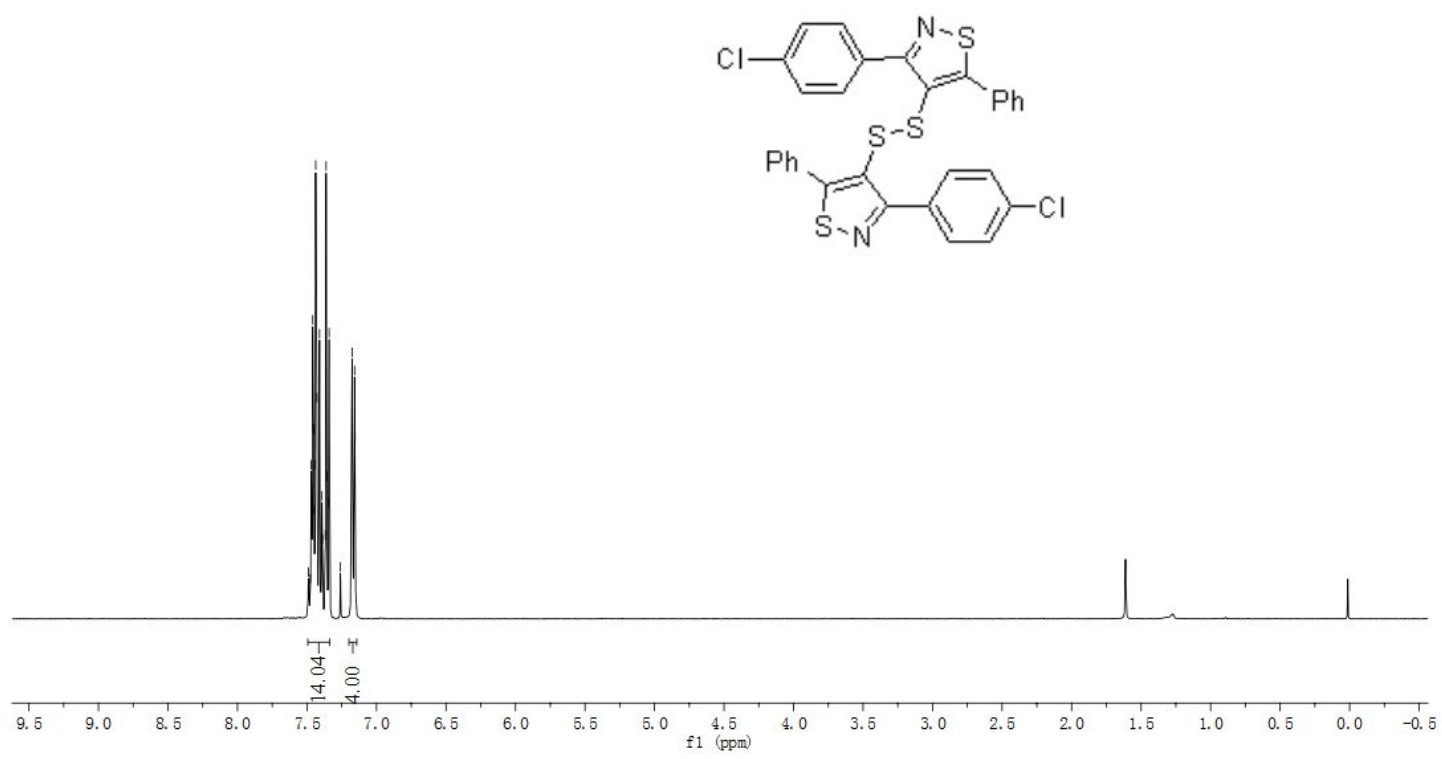

${ }^{1} \mathrm{H}$ NMR $400 \mathrm{MHz}$, in $\mathrm{CDCl}_{3}$ :

1,2-bis(3-(4-chlorophenyl)-5-phenylisothiazol-4-yl)disulfane (16)

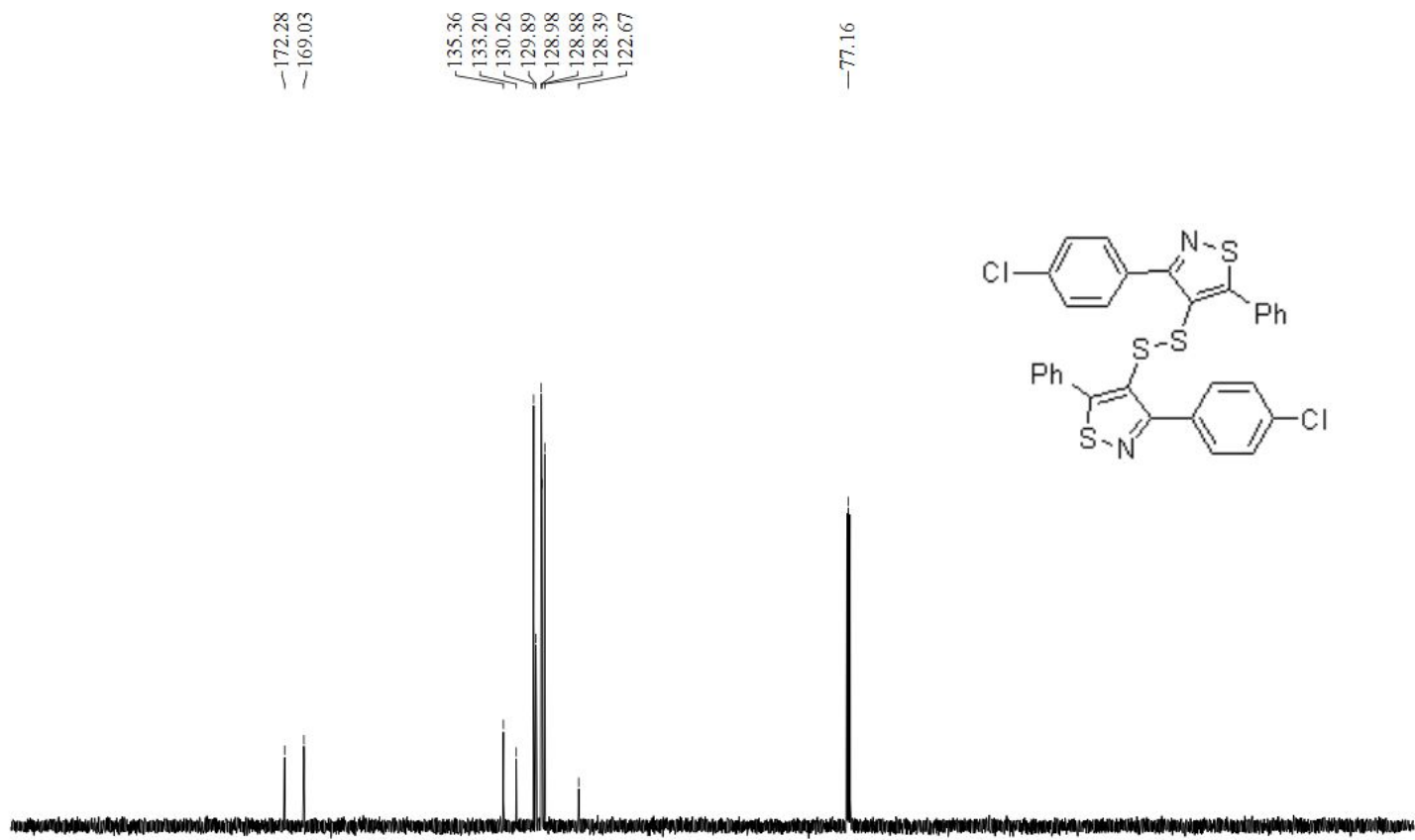

$\begin{array}{lllllllllllllllllllllll}1 & 1 \\ 210 & 200 & 190 & 180 & 170 & 160 & 150 & 140 & 130 & 120 & 110 & 100 & 90 & 80 & 70 & 60 & 50 & 40 & 30 & 20 & 10 & 0 & -10\end{array}$

${ }^{13} \mathrm{C}$ NMR $125 \mathrm{MHz}$, in $\mathrm{CDCl}_{3}$ :

1,2-bis(3-(4-chlorophenyl)-5-phenylisothiazol-4-yl)disulfane (16) 


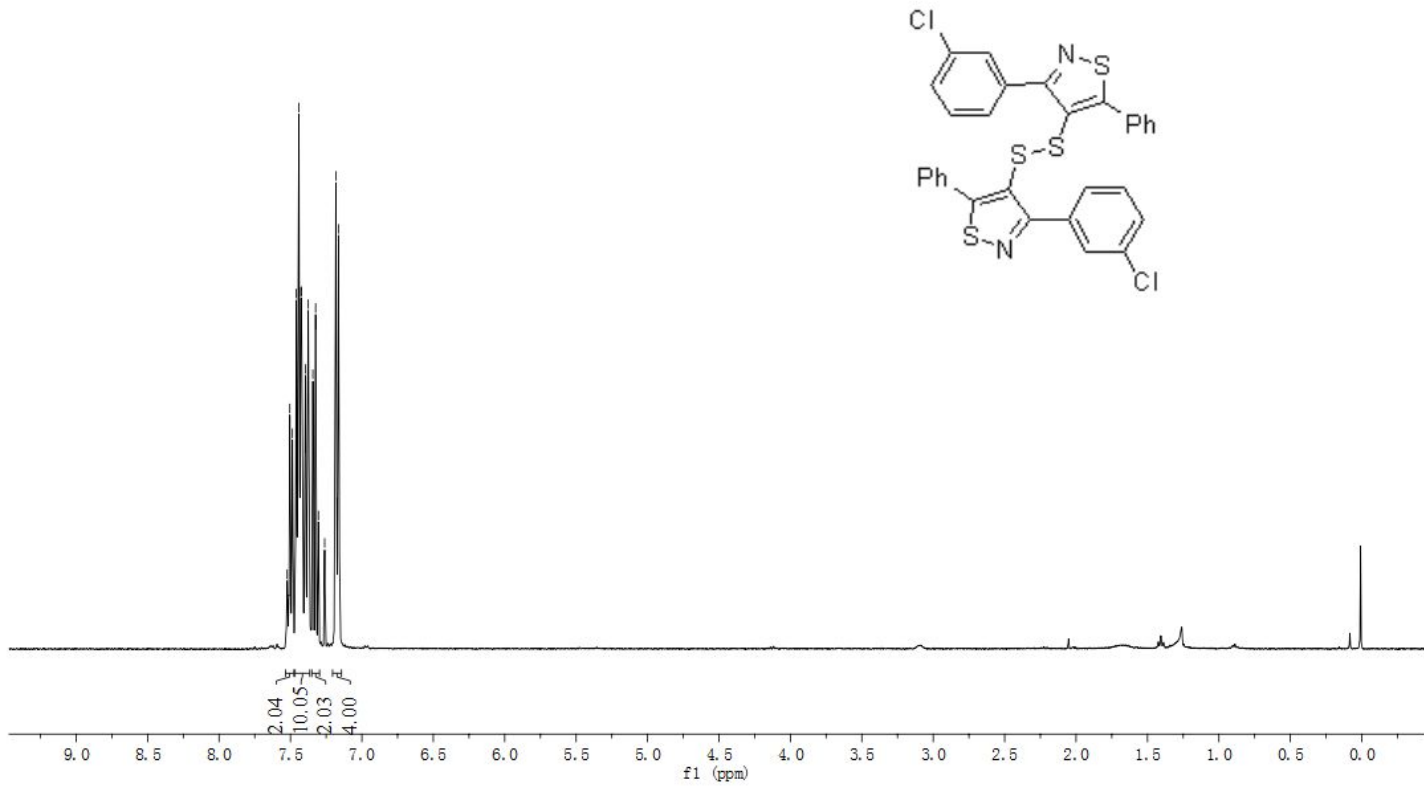

${ }^{1} \mathrm{H}$ NMR $400 \mathrm{MHz}$, in $\mathrm{CDCl}_{3}$ :

1,2-bis(5-(3-chlorophenyl)-3-phenylisothiazol-4-yl)disulfane (17)

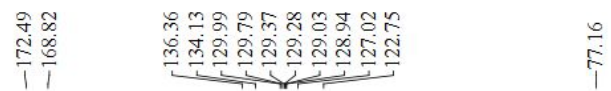

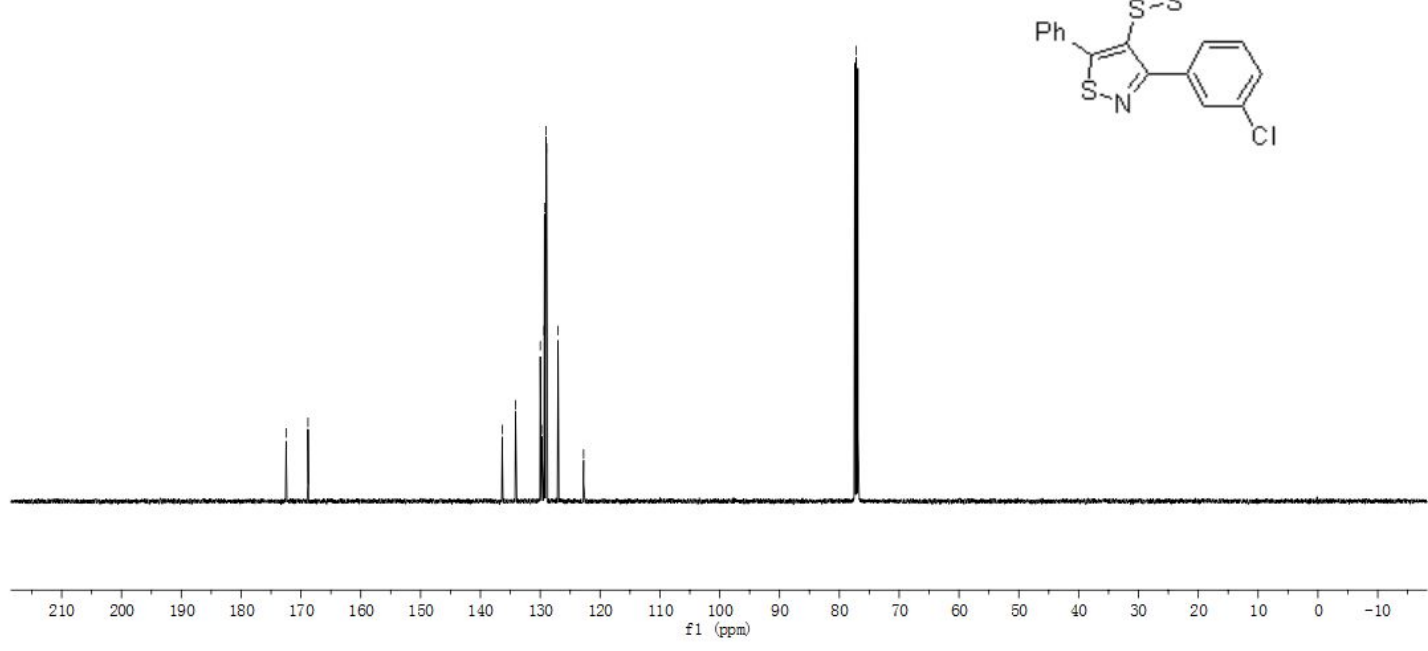

${ }^{13} \mathrm{C}$ NMR $125 \mathrm{MHz}$, in $\mathrm{CDCl}_{3}$ :

1,2-bis(5-(3-chlorophenyl)-3-phenylisothiazol-4-yl)disulfane (17) 

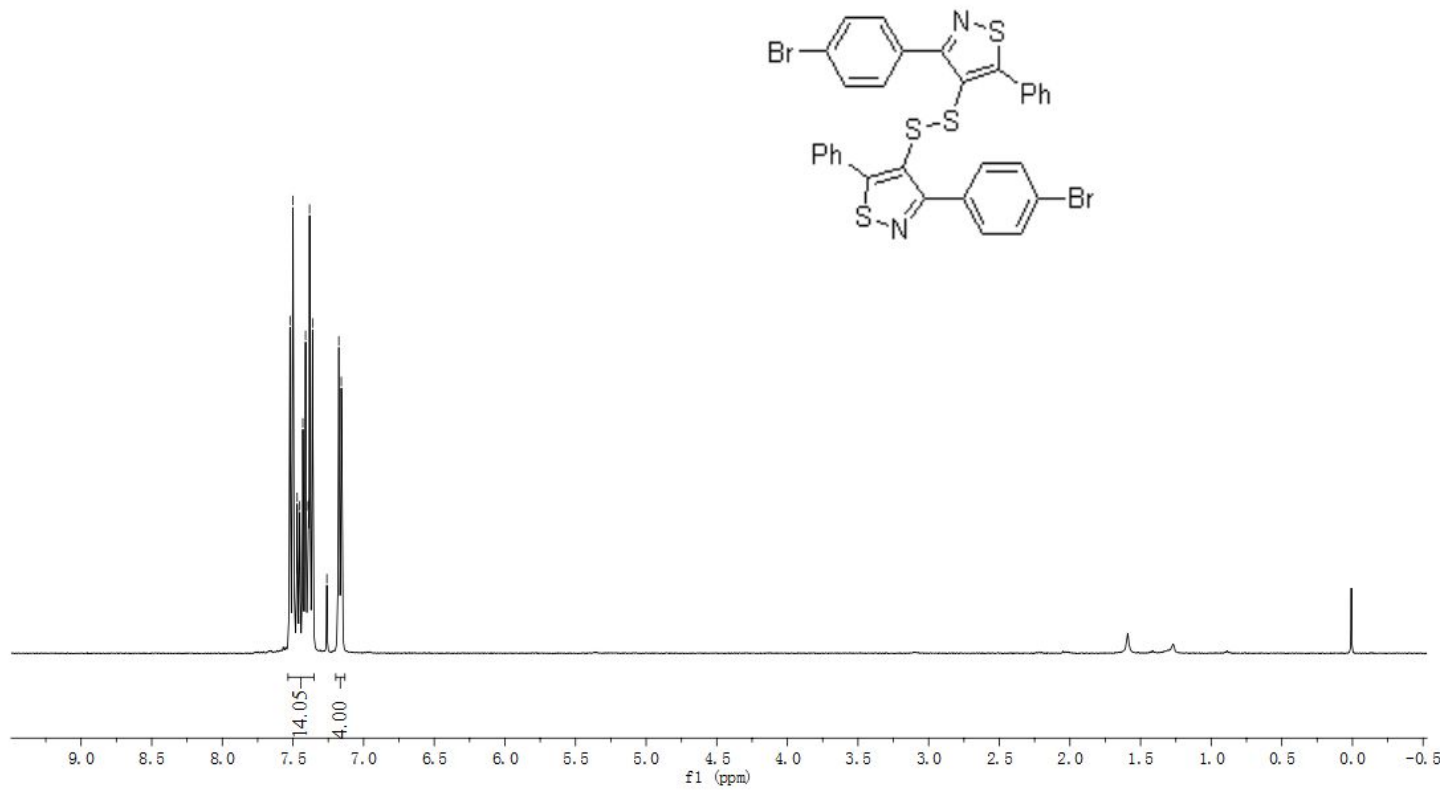

${ }^{1} \mathrm{H}$ NMR $400 \mathrm{MHz}$, in $\mathrm{CDCl}_{3}$ :

1,2-bis(3-(4-bromophenyl)-5-phenylisothiazol-4-yl)disulfane (18)

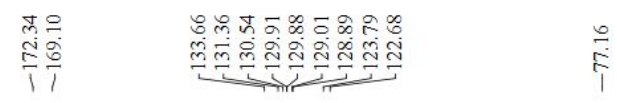

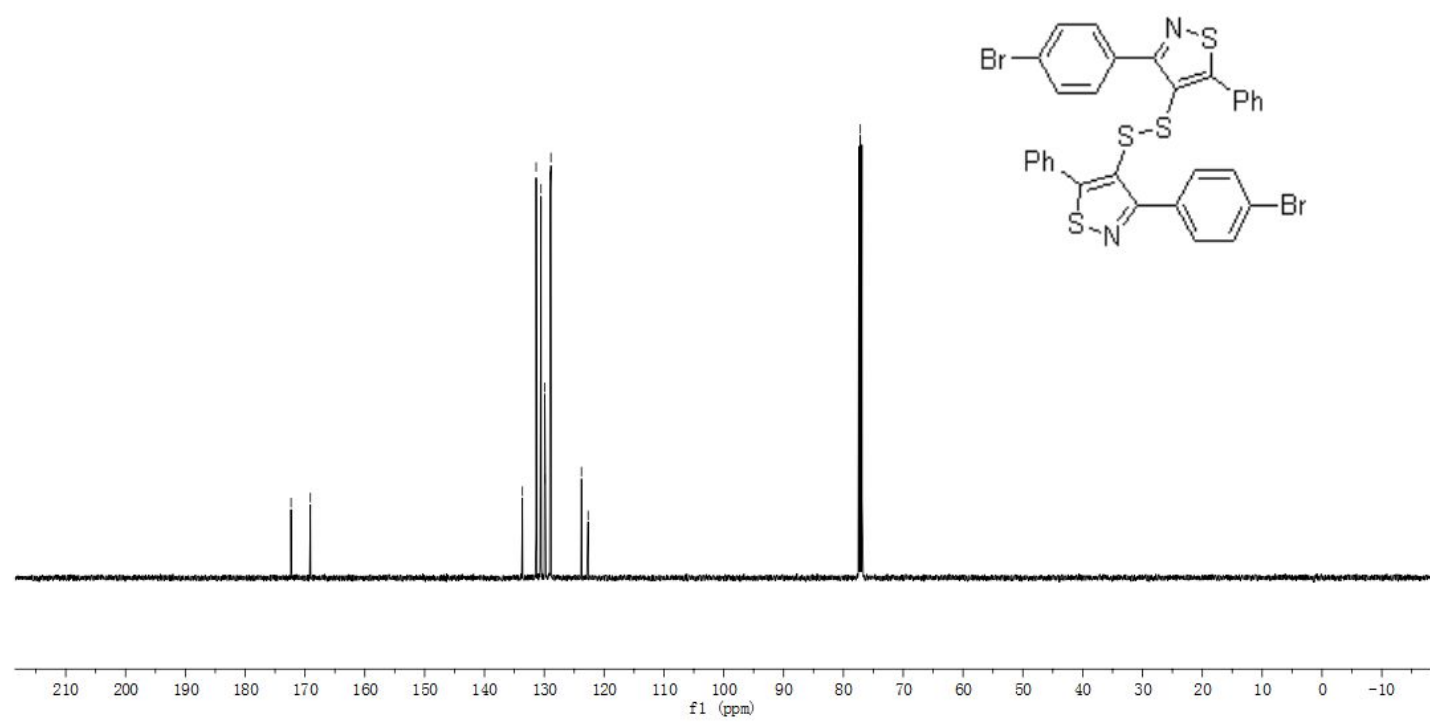

${ }^{13} \mathrm{C}$ NMR $125 \mathrm{MHz}$, in $\mathrm{CDCl}_{3}$ :

1,2-bis(3-(4-bromophenyl)-5-phenylisothiazol-4-yl)disulfane (18) 

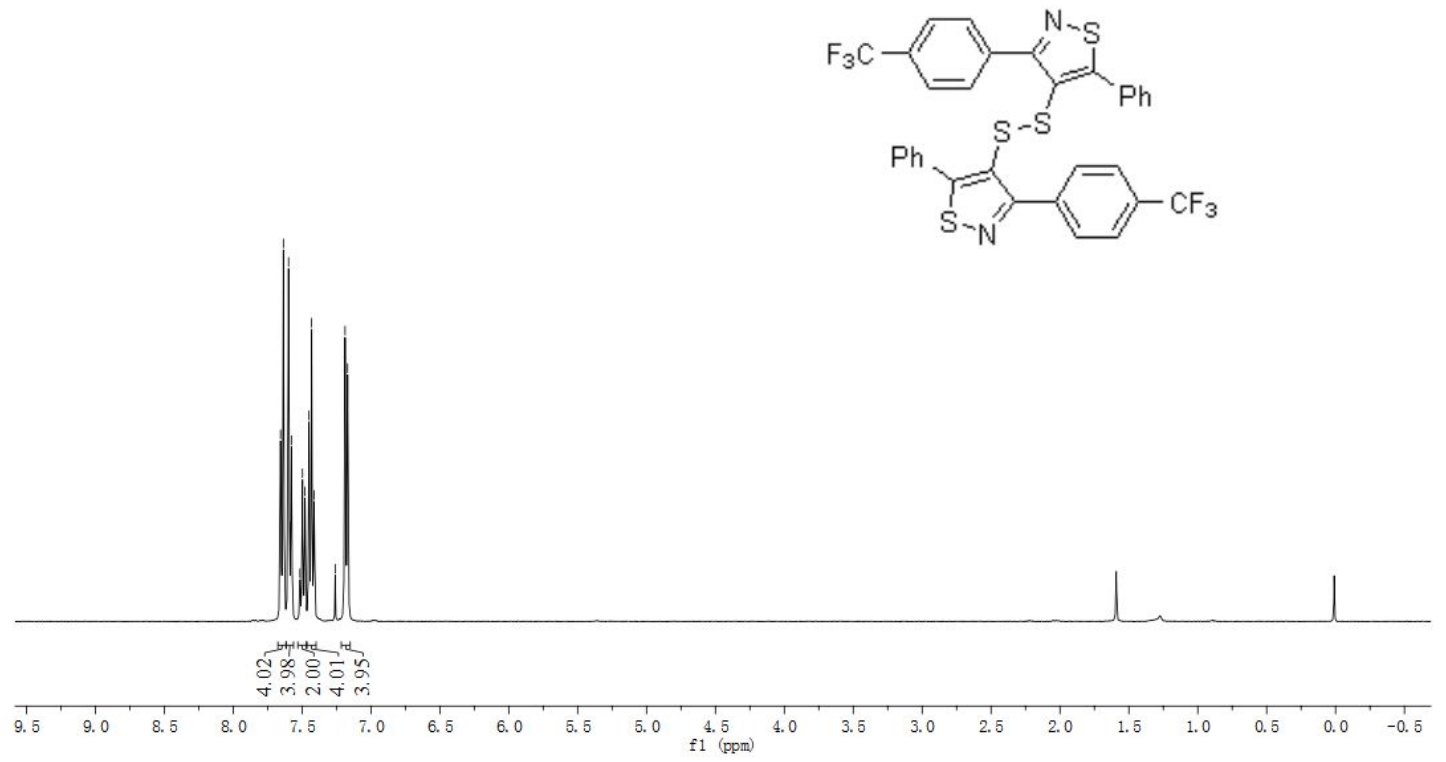

${ }^{1} \mathrm{H}$ NMR $400 \mathrm{MHz}$, in $\mathrm{CDCl}_{3}$ :

1,2-bis(5-phenyl-3-(4-(trifluoromethyl)phenyl)isothiazol-4-yl)disulfane (19)

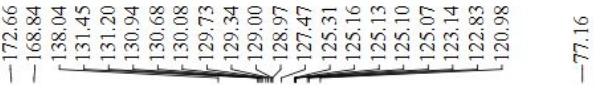

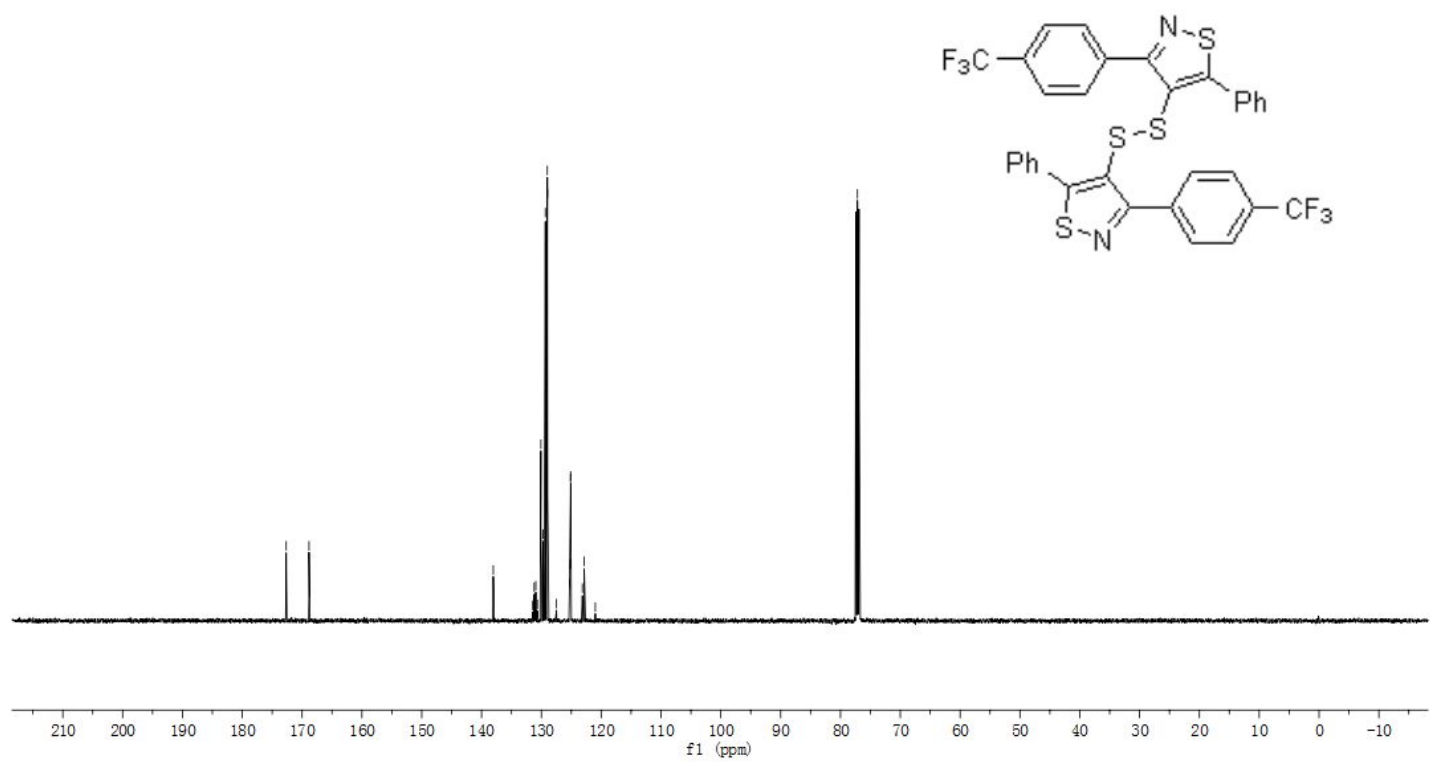

${ }^{13} \mathrm{C}$ NMR $125 \mathrm{MHz}$, in $\mathrm{CDCl}_{3}$ :

1,2-bis(5-phenyl-3-(4-(trifluoromethyl)phenyl)isothiazol-4-yl)disulfane (19) 


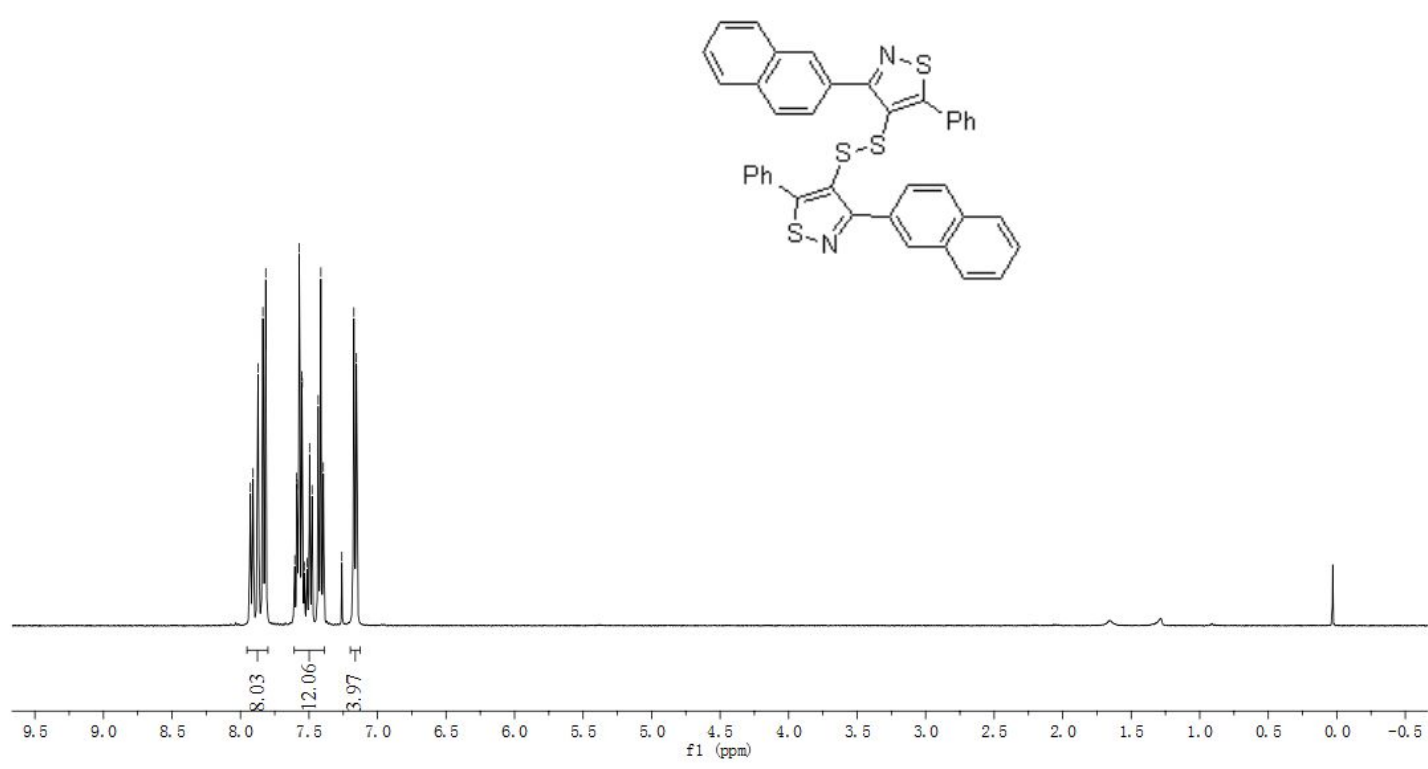

${ }^{1} \mathrm{H}$ NMR $400 \mathrm{MHz}$, in $\mathrm{CDCl}_{3}$ :

1,2-bis(3-(naphthalen-2-yl)-5-phenylisothiazol-4-yl)disulfane (20)

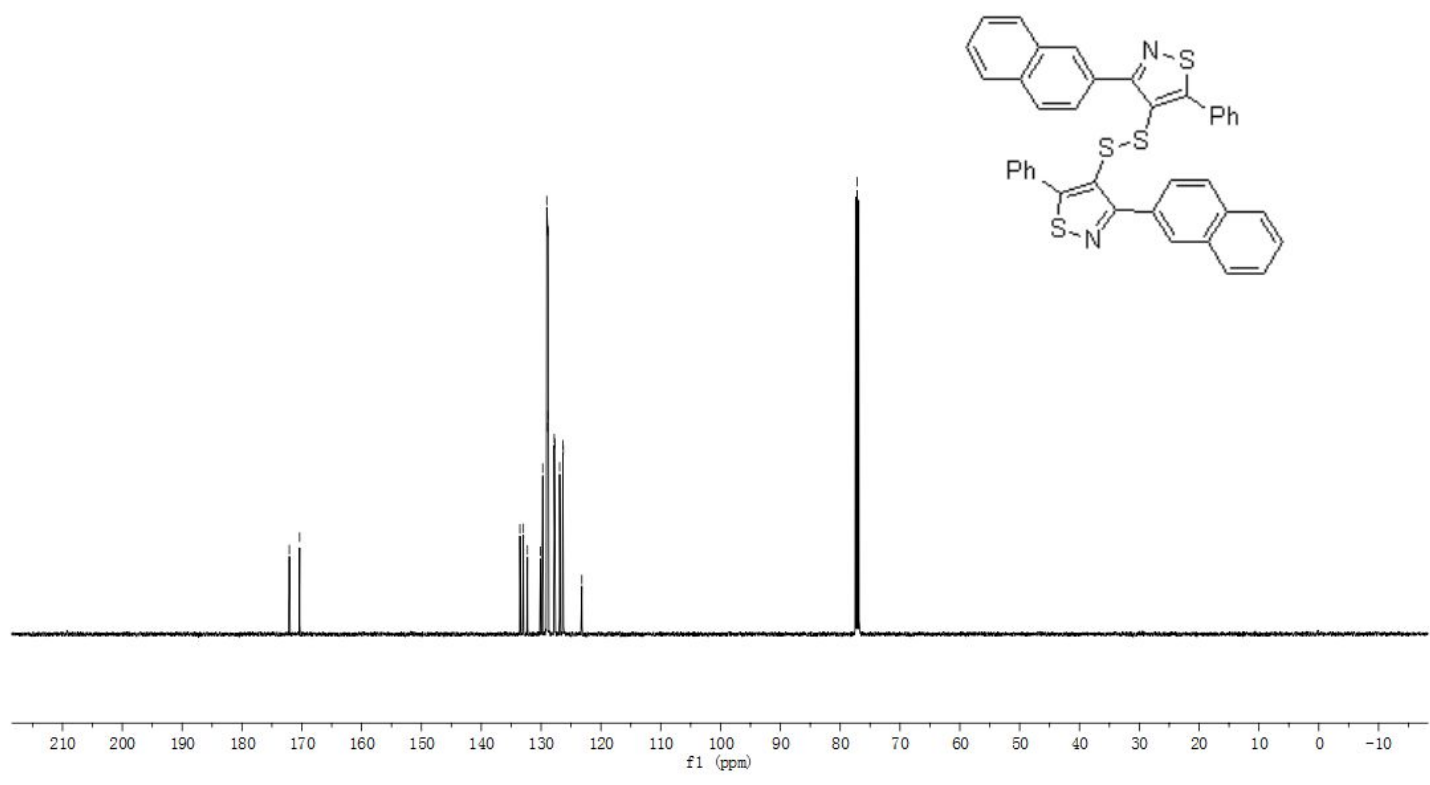

${ }^{13} \mathrm{C}$ NMR $125 \mathrm{MHz}$, in $\mathrm{CDCl}_{3}$ :

1,2-bis(3-(naphthalen-2-yl)-5-phenylisothiazol-4-yl)disulfane (20) 


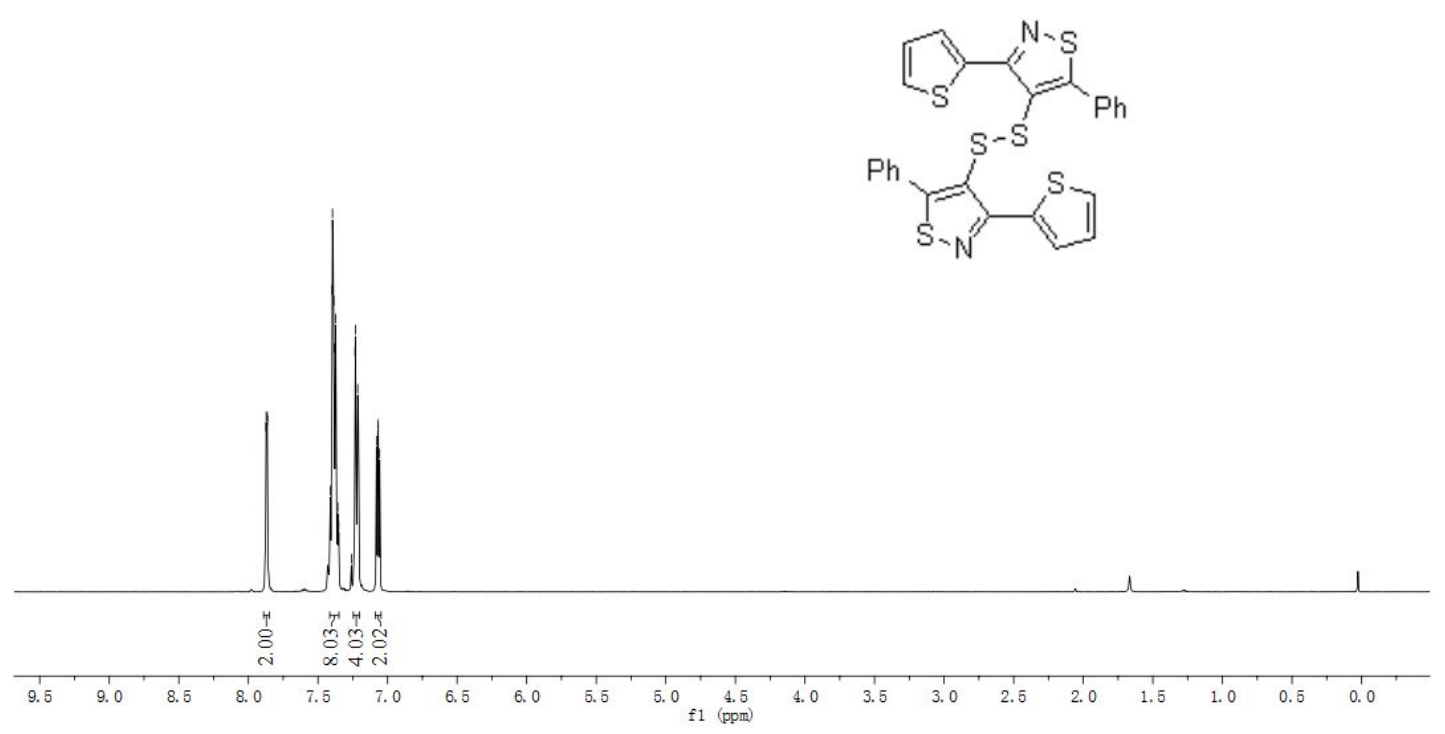

${ }^{1} \mathrm{H}$ NMR $400 \mathrm{MHz}$, in $\mathrm{CDCl}_{3}$ :

1,2-bis(5-phenyl-3-(thiophen-2-yl)isothiazol-4-yl)disulfane (21)
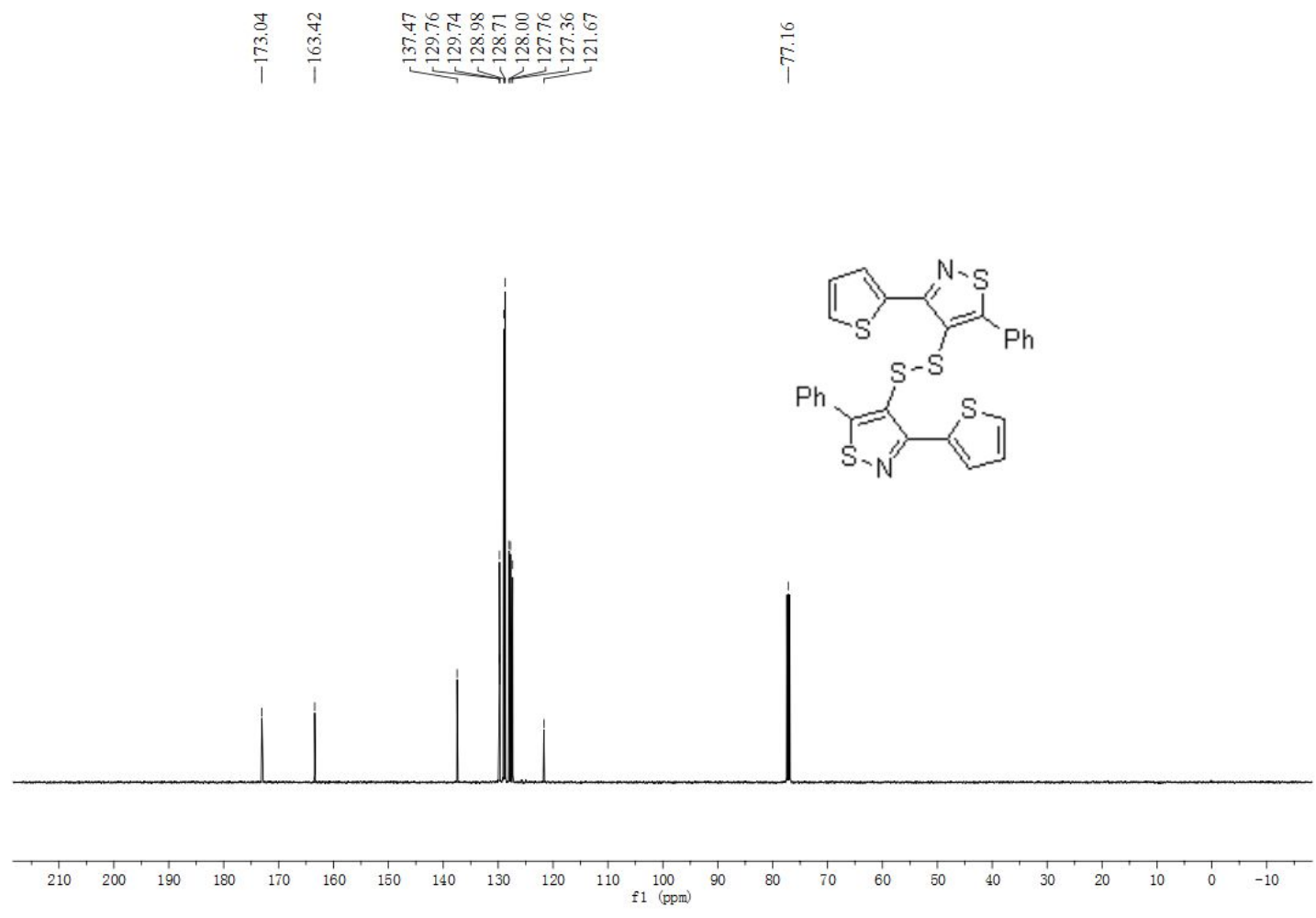

${ }^{13} \mathrm{C}$ NMR $125 \mathrm{MHz}$, in $\mathrm{CDCl}_{3}$ :

1,2-bis(5-phenyl-3-(thiophen-2-yl)isothiazol-4-yl)disulfane (21) 

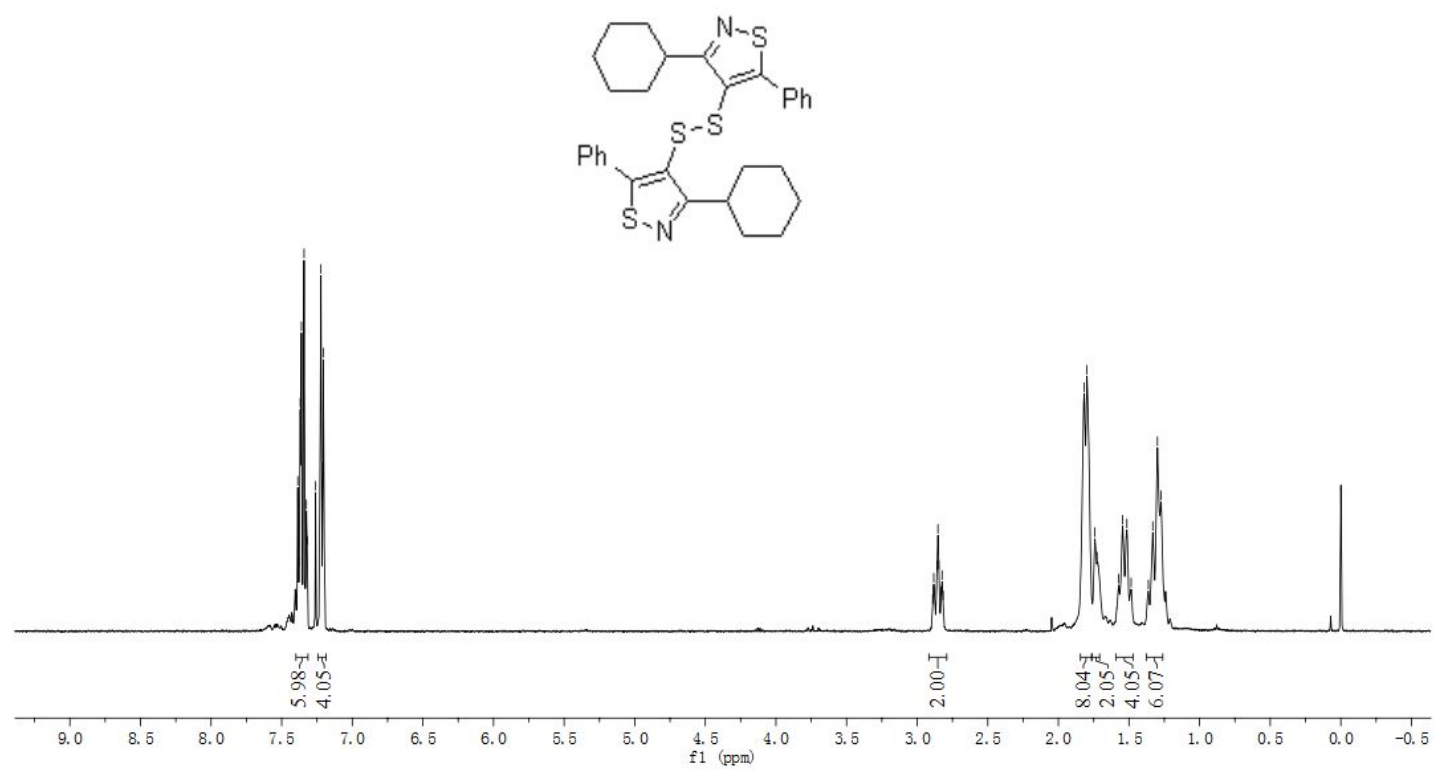

${ }^{1} \mathrm{H}$ NMR $400 \mathrm{MHz}$, in $\mathrm{CDCl}_{3}$ :

1,2-bis(3-cyclohexyl-5-phenylisothiazol-4-yl)disulfane (22)

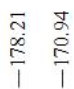

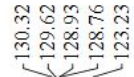
$\stackrel{\frac{6}{6}}{\frac{1}{6}}$

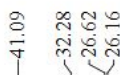

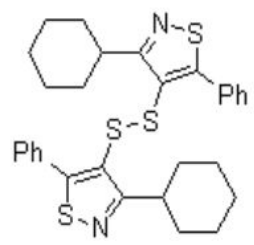

\begin{tabular}{lllllllll}
210 & 200 & 190 & 180 & 170 & 160 & 1 & 1 \\
\hline
\end{tabular}

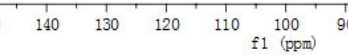

${ }^{13} \mathrm{C}$ NMR $125 \mathrm{MHz}$, in $\mathrm{CDCl}_{3}$ :

1,2-bis(3-cyclohexyl-5-phenylisothiazol-4-yl)disulfane (22) 


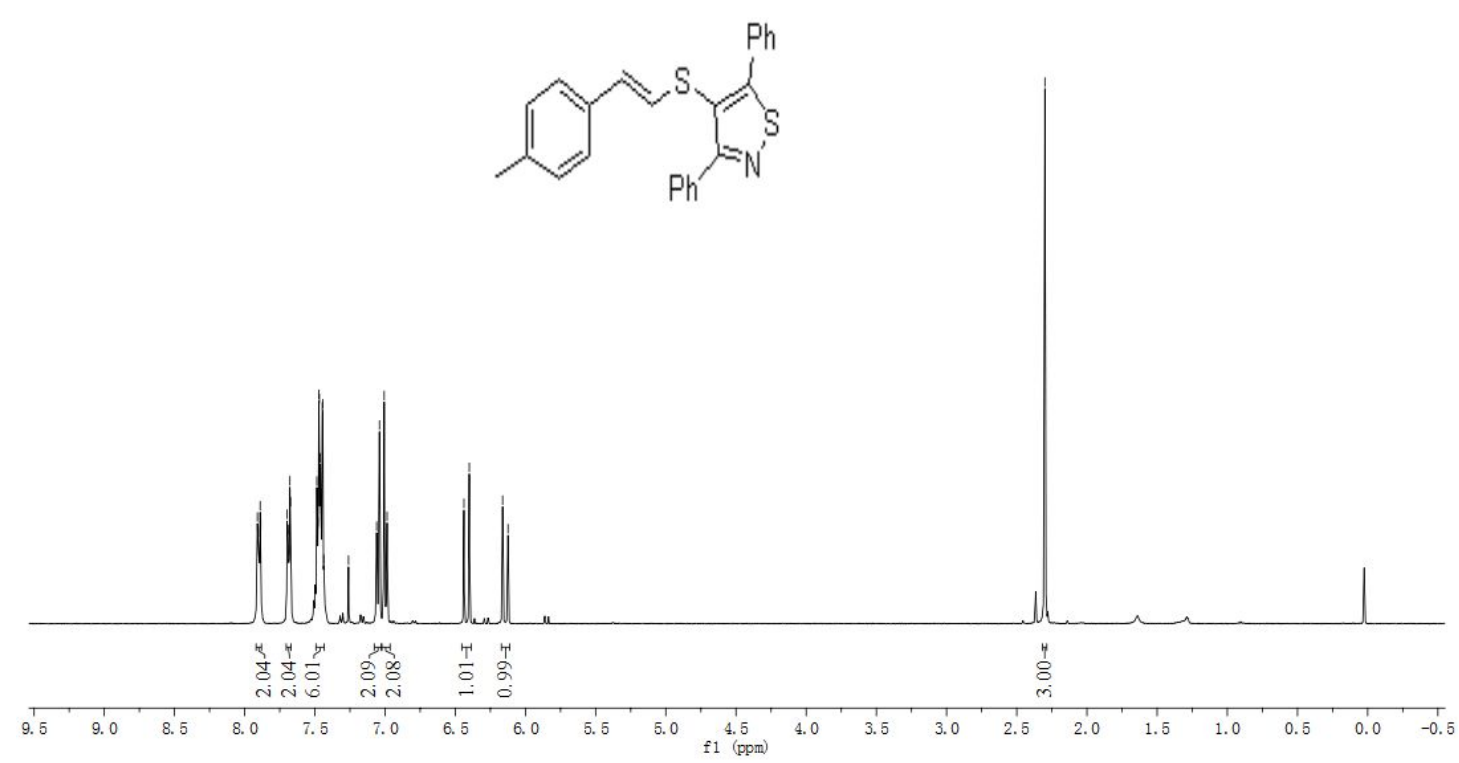

${ }^{1} \mathrm{H}$ NMR $400 \mathrm{MHz}$, in $\mathrm{CDCl}_{3}$ :

(E)-4-((4-methylstyryl)thio)-3,5-diphenylisothiazole (23)

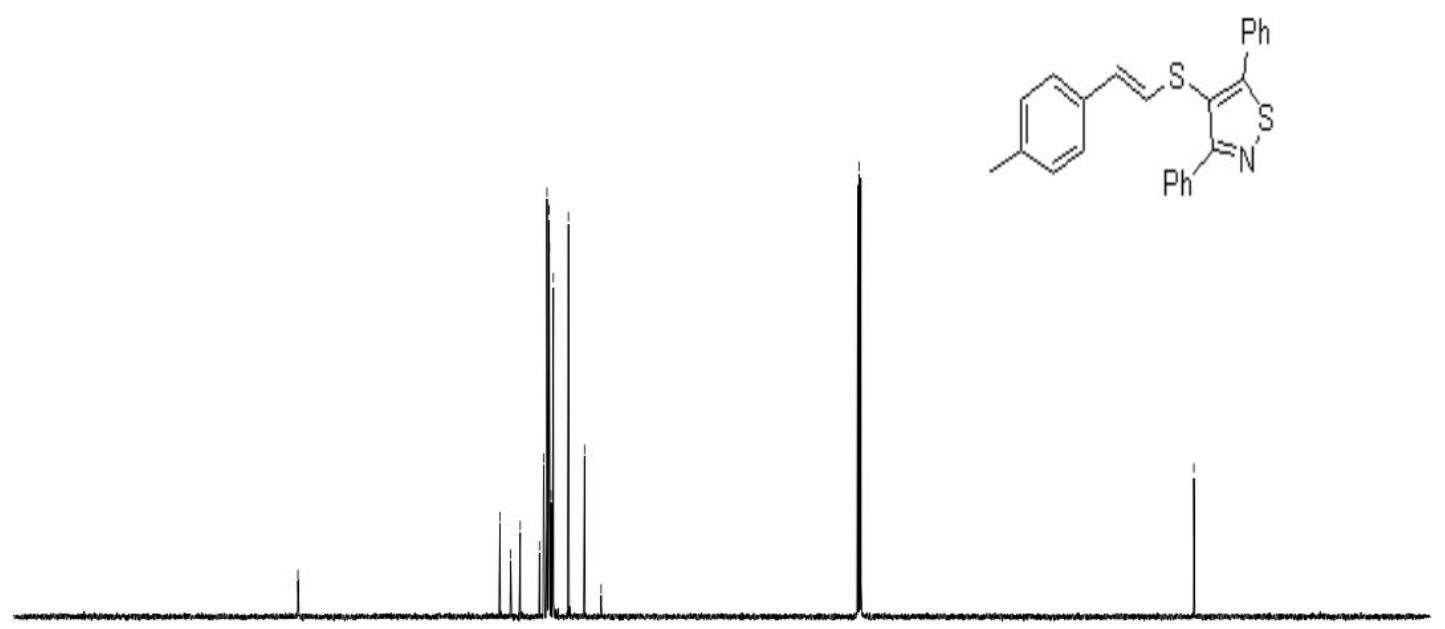

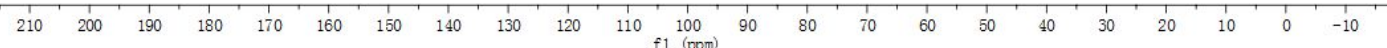

${ }^{13} \mathrm{C}$ NMR $125 \mathrm{MHz}$, in $\mathrm{CDCl}_{3}$ :

(E)-4-((4-methylstyryl)thio)-3,5-diphenylisothiazole (23) 


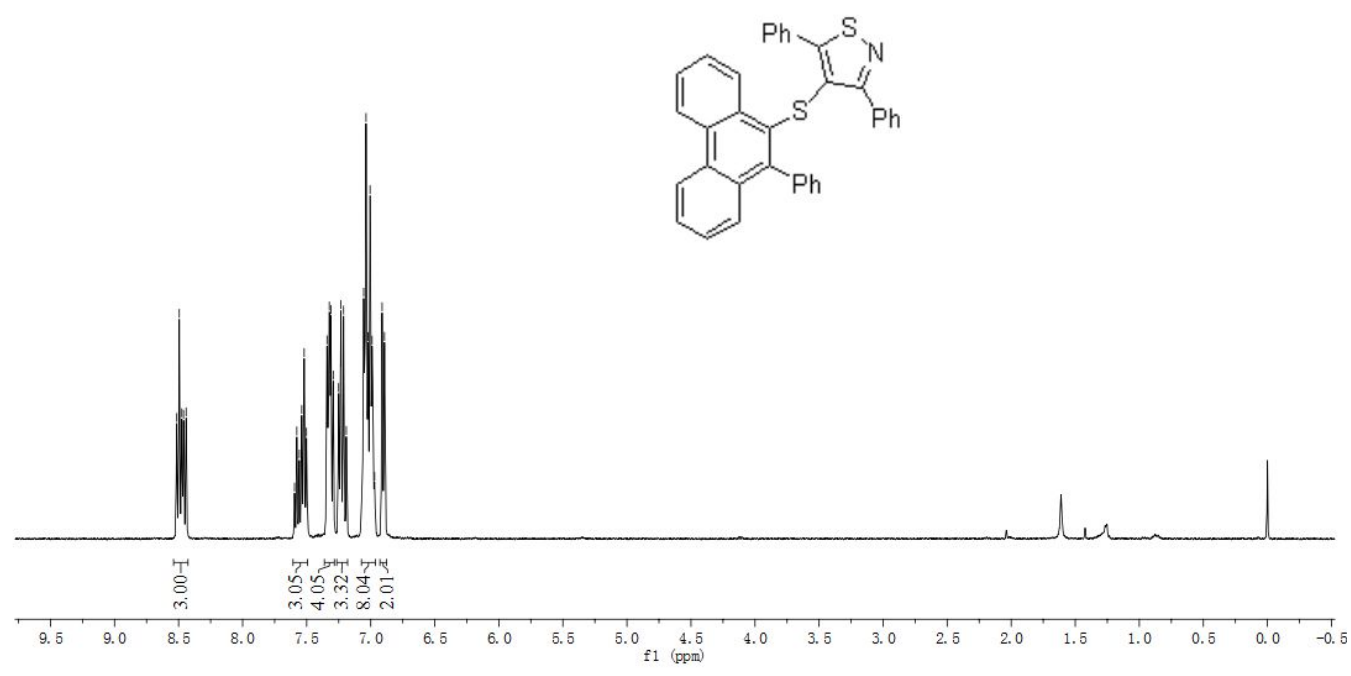

${ }^{1} \mathrm{H}$ NMR $400 \mathrm{MHz}$, in $\mathrm{CDCl}_{3}$ :

3,5-diphenyl-4-((10-phenylphenanthren-9-yl)thio)isothiazole (24)

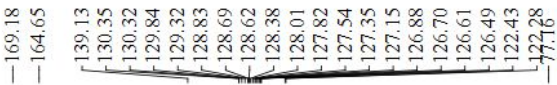

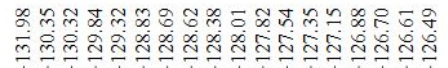

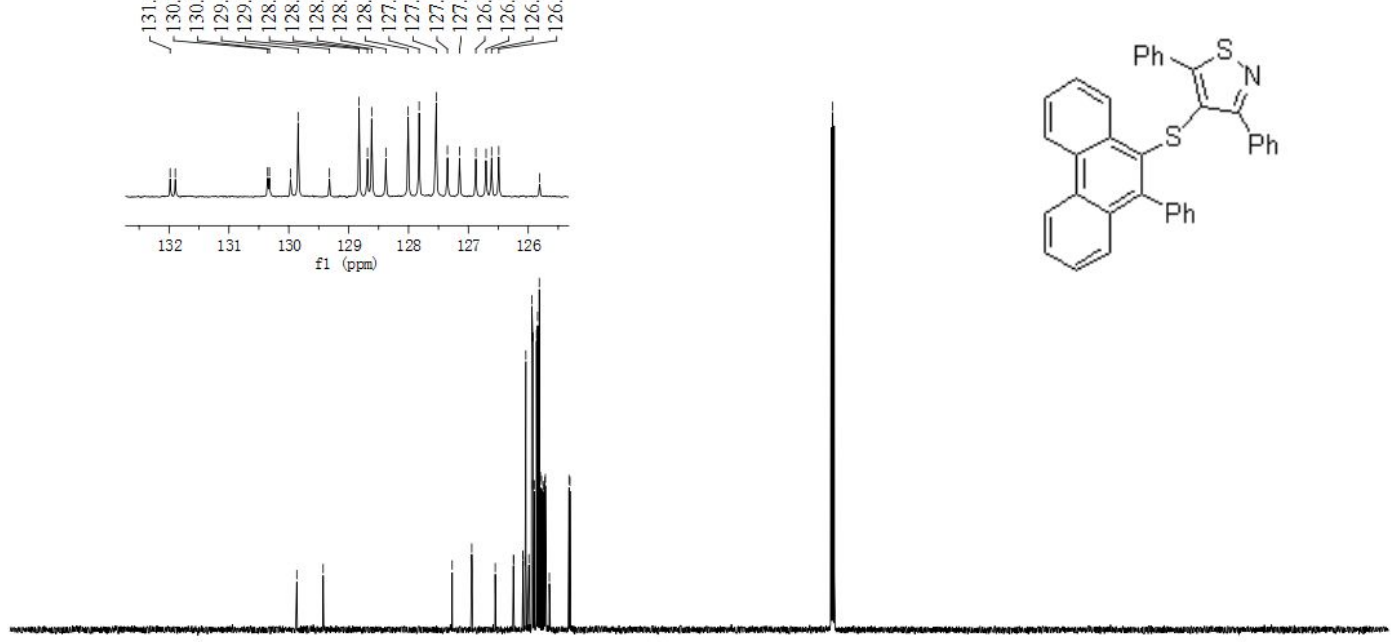

$\begin{array}{lllllllllllllllllllllll}210 & 200 & 190 & 180 & 170 & 160 & 150 & 140 & 130 & 120 & 110 & 100 & 90 & 80 & 70 & 60 & 50 & 40 & 30 & 20 & 10 & 0 & -10\end{array}$

${ }^{13} \mathrm{C}$ NMR $125 \mathrm{MHz}$, in $\mathrm{CDCl}_{3}$ :

3,5-diphenyl-4-((10-phenylphenanthren-9-yl)thio)isothiazole (24) 

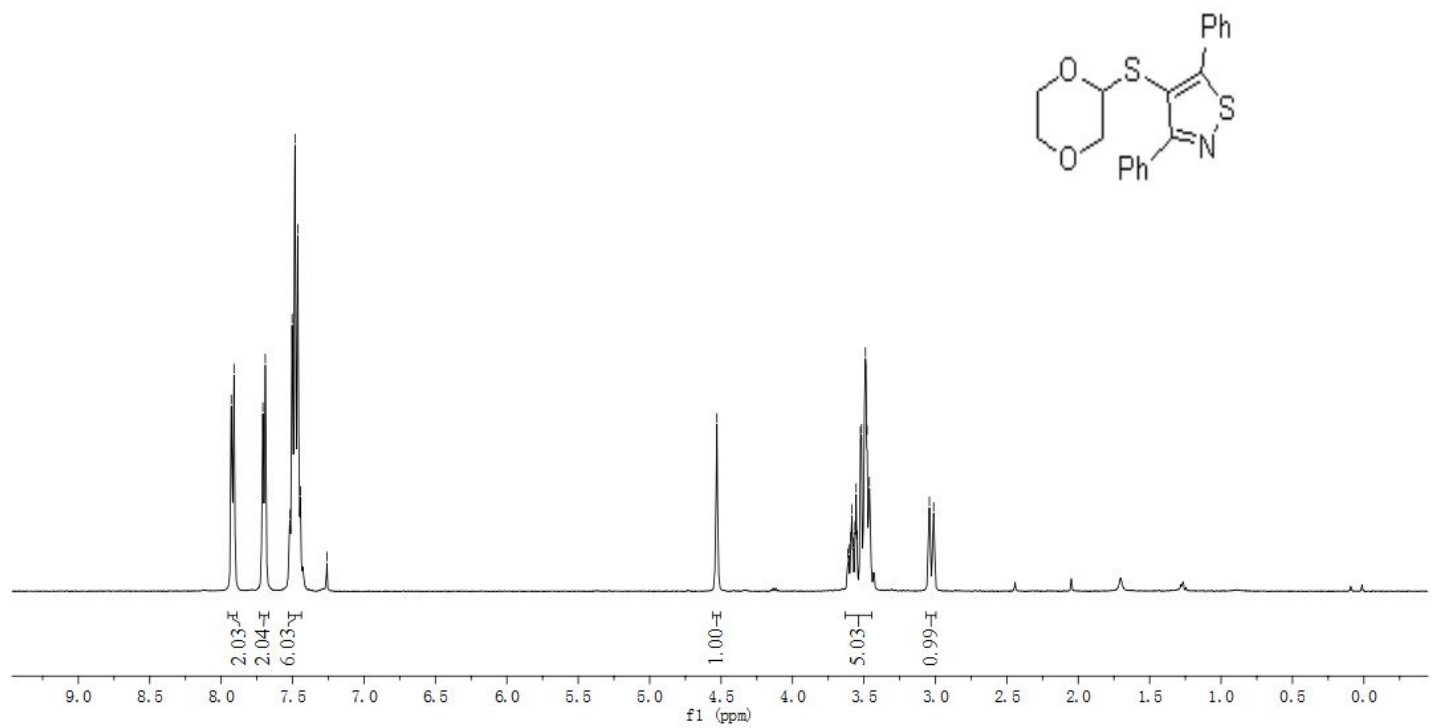

${ }^{1} \mathrm{H}$ NMR $400 \mathrm{MHz}$, in $\mathrm{CDCl}_{3}$ : 4-((1,4-dioxan-2-yl)thio)-3,5-diphenylisothiazole

(25)

\begin{tabular}{|c|c|}
\hline 产总 & 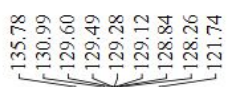 \\
\hline
\end{tabular}

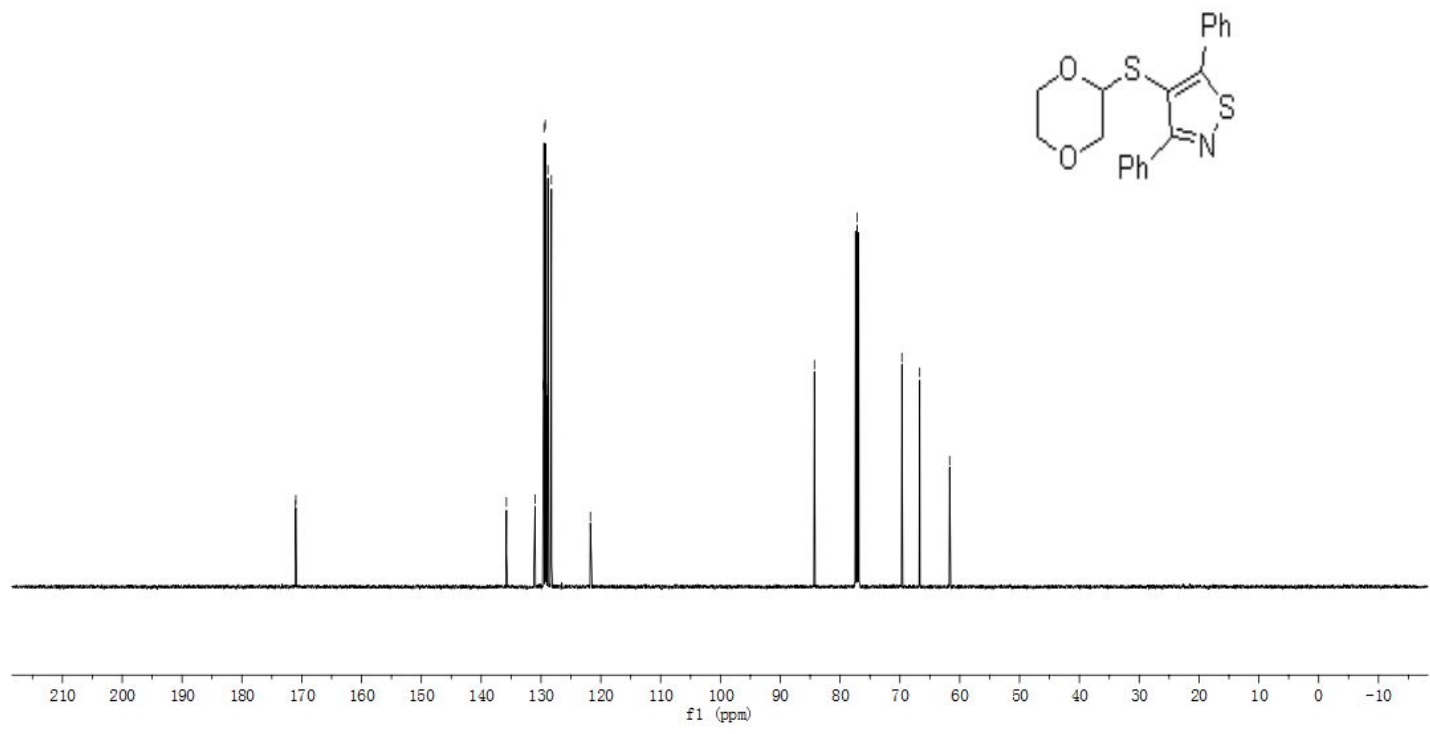

${ }^{13} \mathrm{C}$ NMR $125 \mathrm{MHz}$, in $\mathrm{CDCl}_{3}$ : 4-((1,4-dioxan-2-yl)thio)-3,5-diphenylisothiazole 

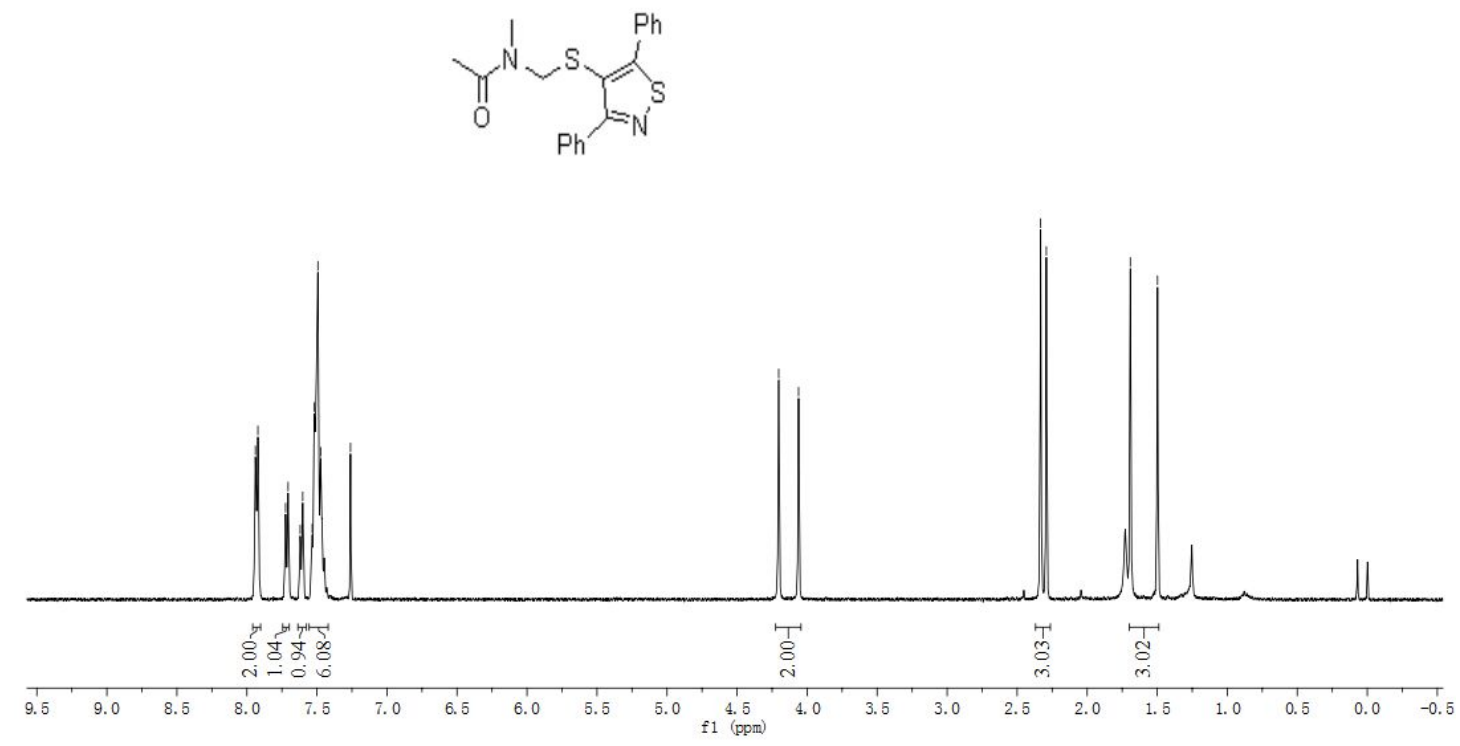

${ }^{1} \mathrm{H}$ NMR $400 \mathrm{MHz}$, in $\mathrm{CDCl}_{3}$ :

N-(((3,5-diphenylisothiazol-4-yl)thio)methyl)-N-methylacetamide (26)

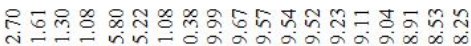

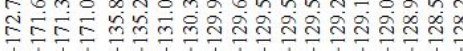

है कूष
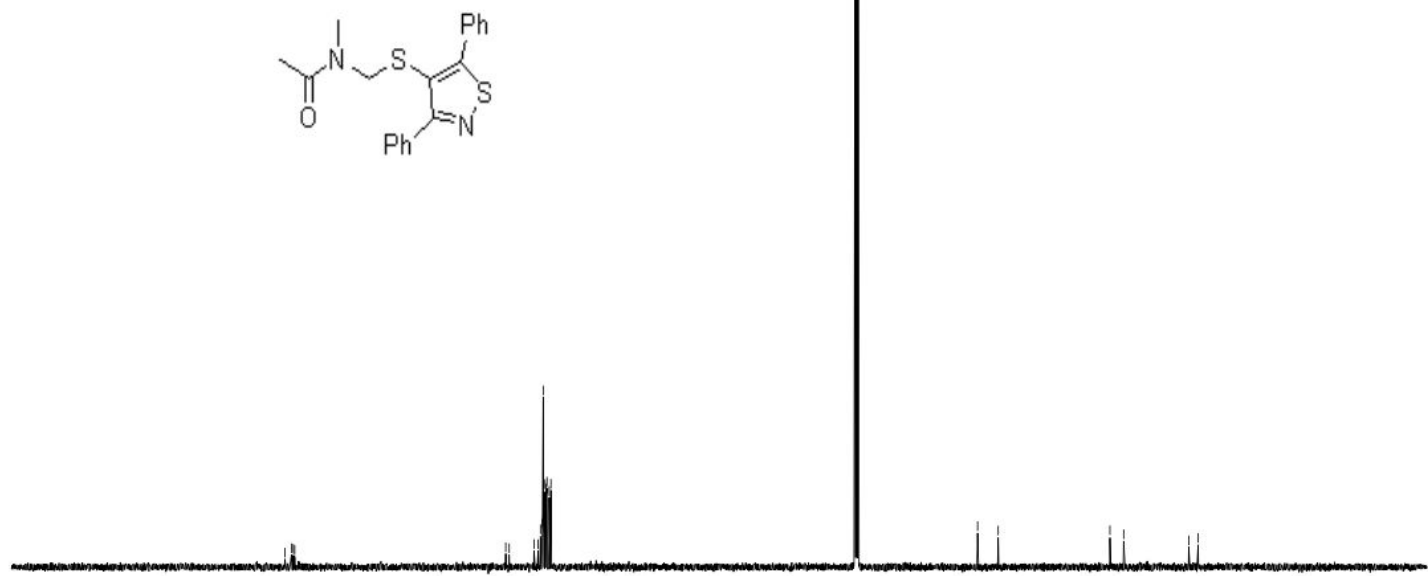

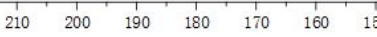

${ }^{13} \mathrm{C}$ NMR $125 \mathrm{MHz}$, in $\mathrm{CDCl}_{3}$ :

$\mathrm{N}$-(((3,5-diphenylisothiazol-4-yl)thio)methyl)-N-methylacetamide (26) 


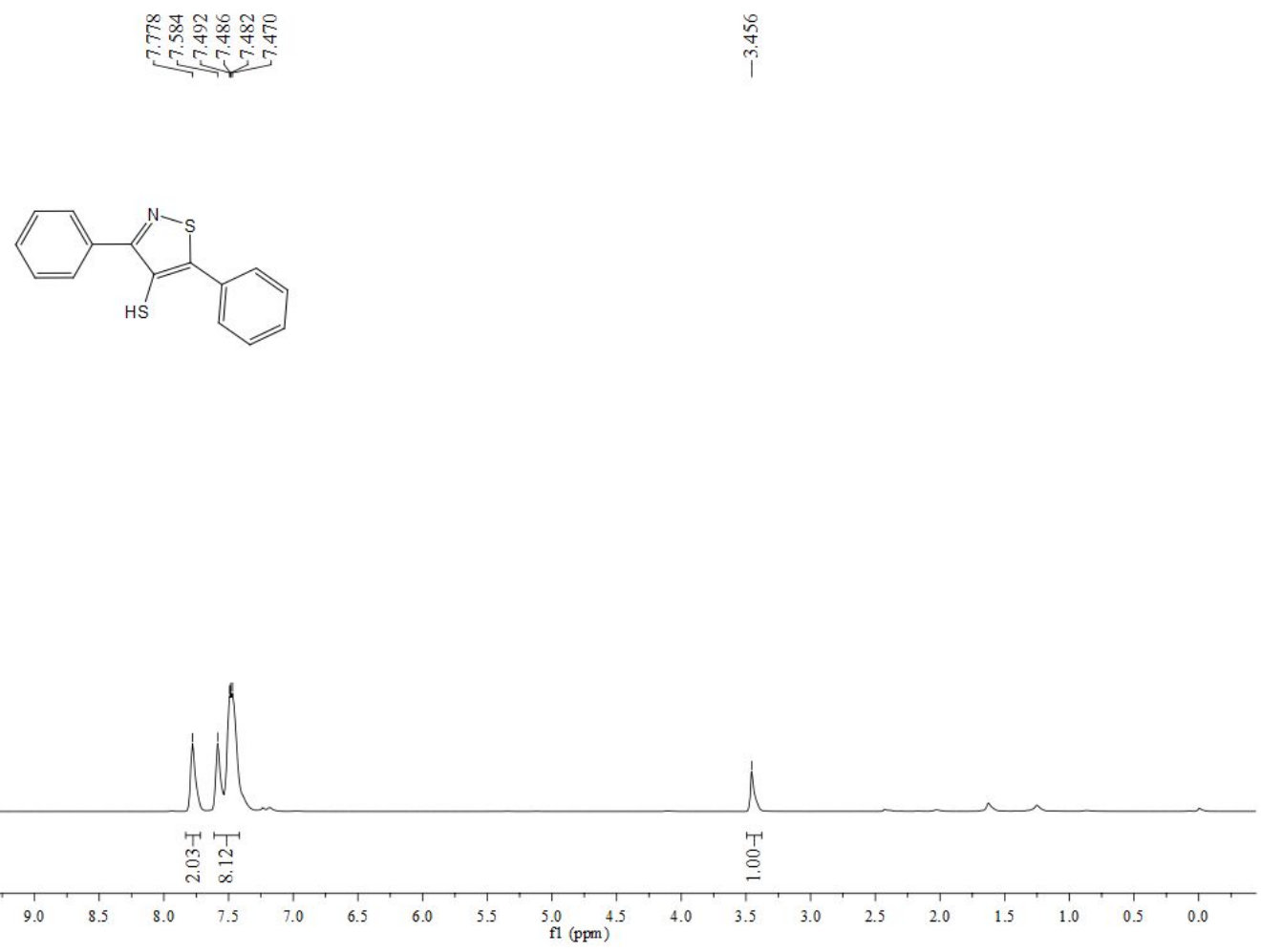

${ }^{1} \mathrm{H}$ NMR $500 \mathrm{MHz}$, in $\mathrm{CDCl}_{3}$ : 3,5-diphenylisothiazole-4-thiol (27)

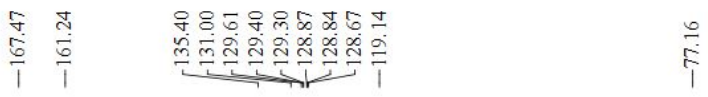
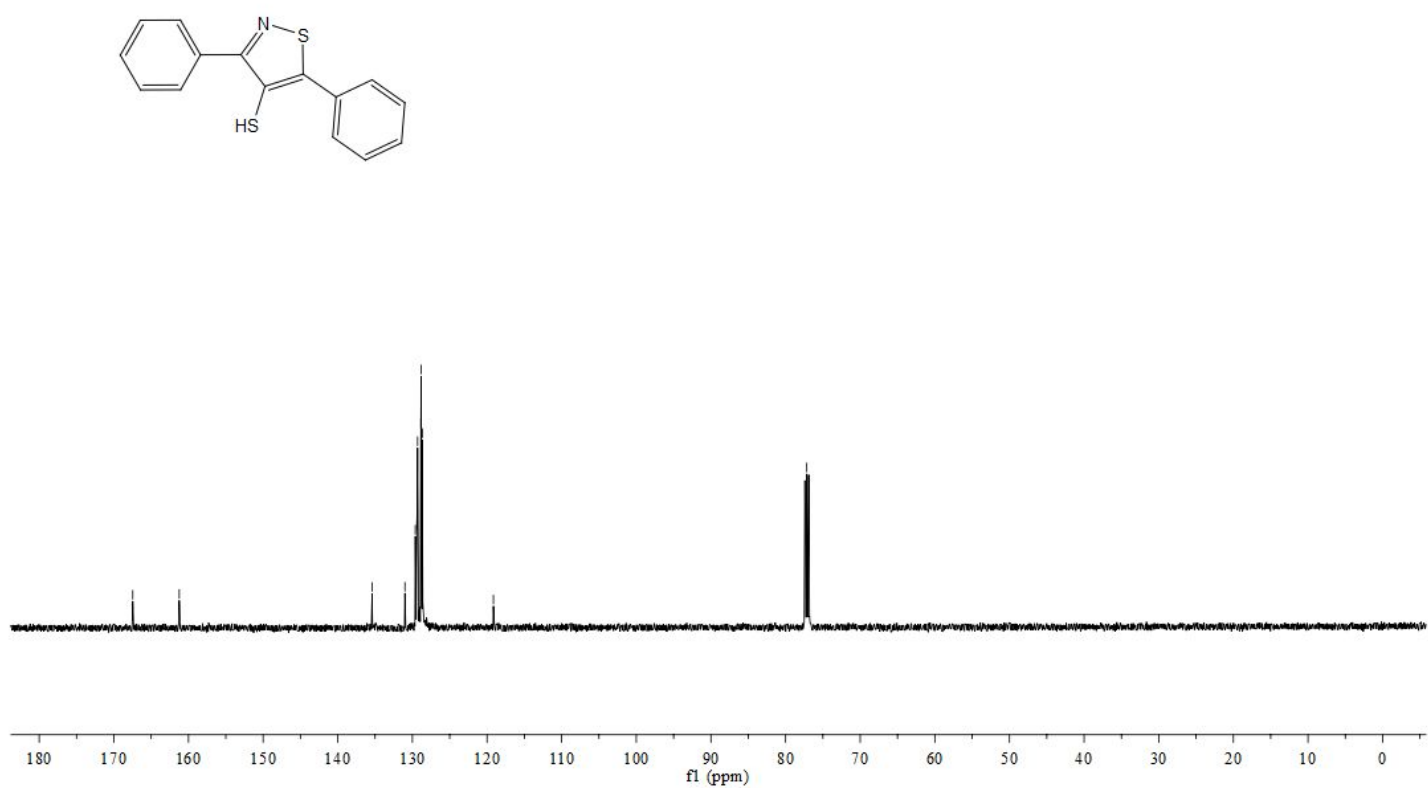

${ }^{13} \mathrm{C}$ NMR $125 \mathrm{MHz}$, in $\mathrm{CDCl}_{3}$ : 3,5-diphenylisothiazole-4-thiol (27) 


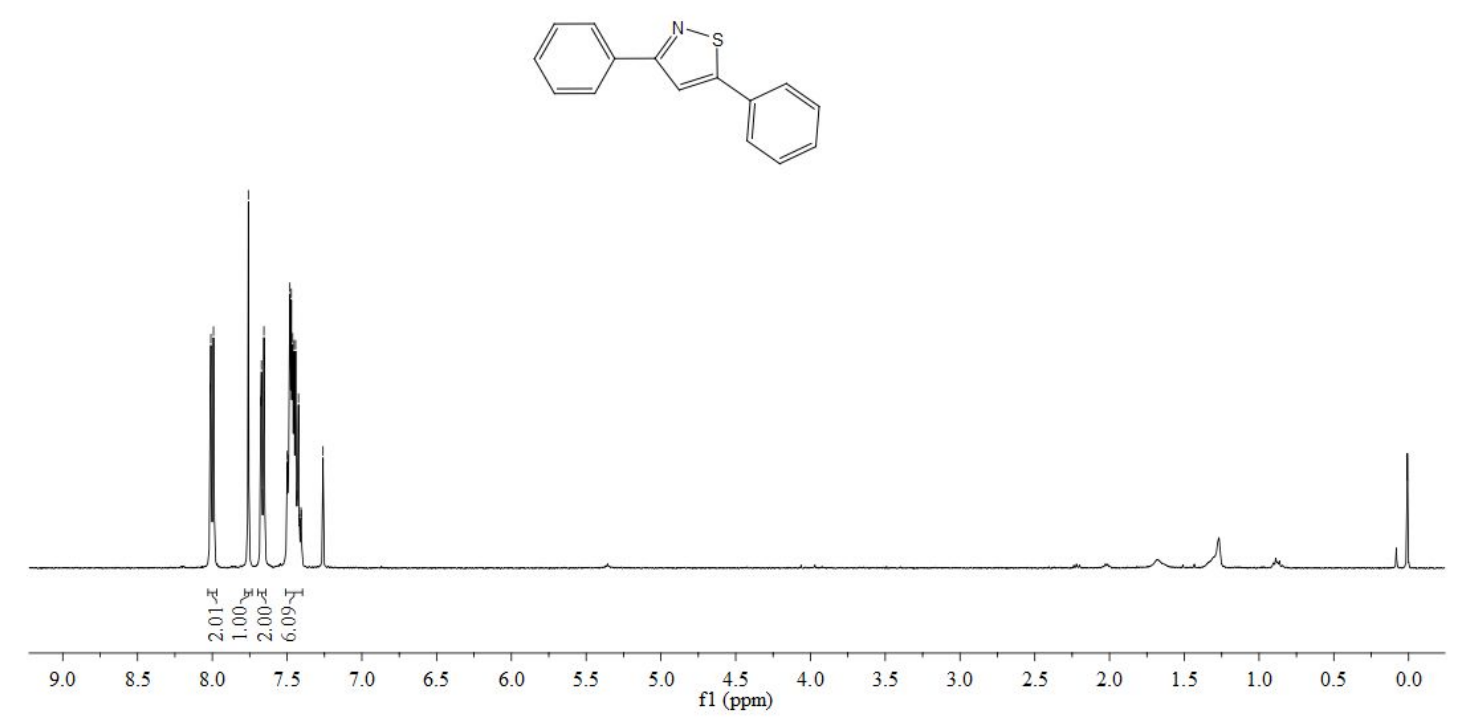

${ }^{1} \mathrm{H}$ NMR $400 \mathrm{MHz}$, in $\mathrm{CDCl}_{3}$ : 3,5-diphenylisothiazole (28) 


\section{X-ray crystal structure and data of compound 3}

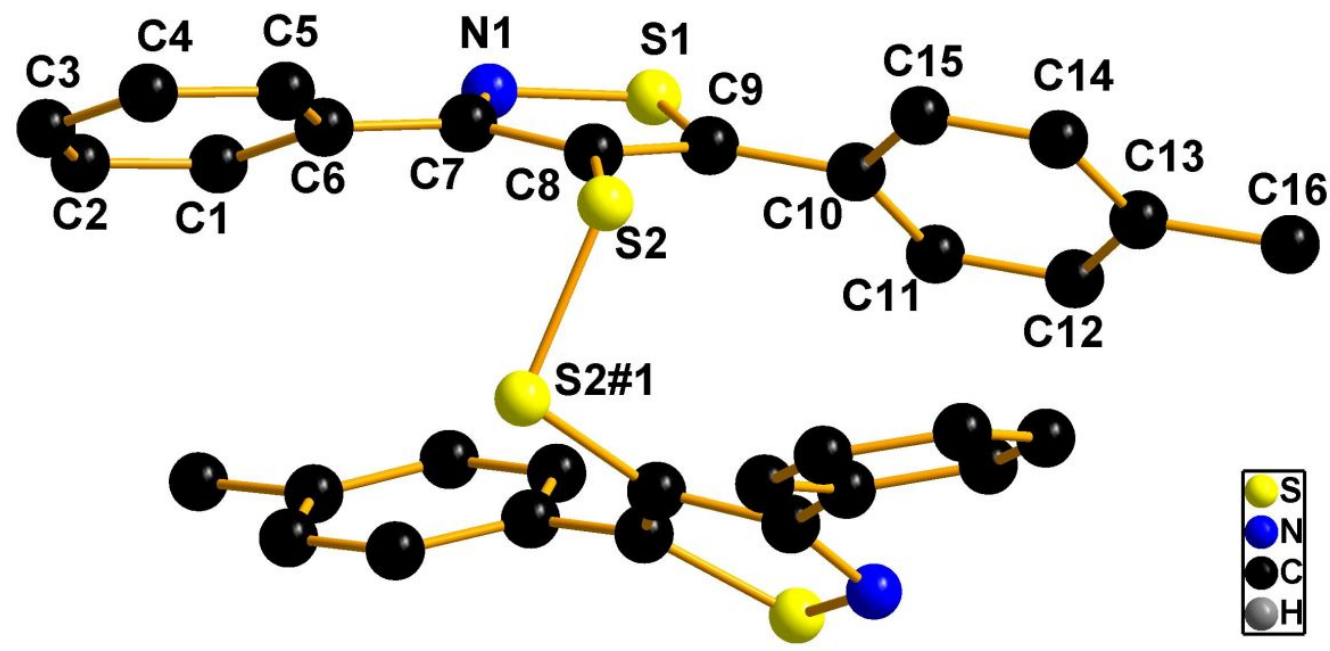

The displacement ellipsoids are drawn at the $30 \%$ probability level

Single crystals suitable for X-ray analysis were obtained by slow evaporation of $\mathrm{CH}_{2} \mathrm{Cl}_{2}$ solvent.

Supplementary crystallographic data was deposited at the Cambridge Crystallographic Data Centre (CCDC) under the number CCDC-1971983 (3) and can be obtained free of charge via www.ccdc.cam.ac.uk/data_request.cif. 
Table S2: Crystal Structure and Data Refinement Parameters of compound 3

\begin{tabular}{|c|c|}
\hline Compound & 3 \\
\hline Empirical Formula & $\mathrm{C}_{16} \mathrm{H}_{12} \mathrm{~N} \mathrm{~S}_{2}$ \\
\hline Formula Weight & 282.39 \\
\hline Crystal System / Space Group & Monoclinic, C 2/c \\
\hline $\mathrm{a} / \mathrm{pm}$ & $1889.4(8)$ \\
\hline $\mathrm{b} / \mathrm{pm}$ & $1288.6(6)$ \\
\hline $\mathrm{c} / \mathrm{pm}$ & $1147.9(5)$ \\
\hline$\alpha /{ }^{\circ}$ & 90 \\
\hline$\beta / \circ$ & $92.493(9)$ \\
\hline$\gamma / \circ$ & 90 \\
\hline $\mathrm{V} / \mathrm{nm}^{3}$ & $2.79(1)$ \\
\hline $\mathrm{Z}$ & 8 \\
\hline$D_{\text {calc }}\left(\mathrm{g} / \mathrm{cm}^{3}\right)$ & 1.344 \\
\hline$\mu\left(\mathrm{mm}^{-1}\right)$ & 0.365 \\
\hline Crystal size (mm) & $0.280 \times 0.250 \times 0.230$ \\
\hline Color / Shape & red \\
\hline Temp $(\mathrm{K})$ & $296(2)$ \\
\hline Theta range for collection & 1.914 to $25.998^{\circ}$ \\
\hline Reflections collected & 11057 \\
\hline Independent reflections & 2725 \\
\hline Data/restraints/parameters & $2725 / 0 / 173$ \\
\hline Goodness of fit on $\mathrm{F}^{2}$ & 1.081 \\
\hline Final $R$ indices $[\mathrm{I}>2 \sigma(\mathrm{I})]$ & $\mathrm{R} 1=0.0552, \mathrm{wR} 2=0.1967$ \\
\hline $\mathrm{R}$ indices (all data) & $\mathrm{R} 1=0.0664, \mathrm{wR} 2=0.2083$ \\
\hline Largest difference peak/hole & 0.380 and -0.283 \\
\hline
\end{tabular}

